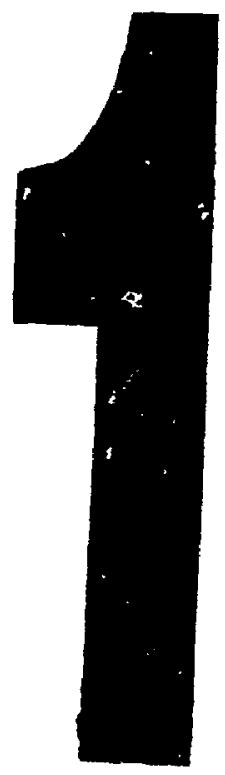

PM-1 31/2"X4" PHOTOGRAPHIC MICROCOPY TARGET NBS 1010a ANSI/ISO \#2 EOUIVALENT

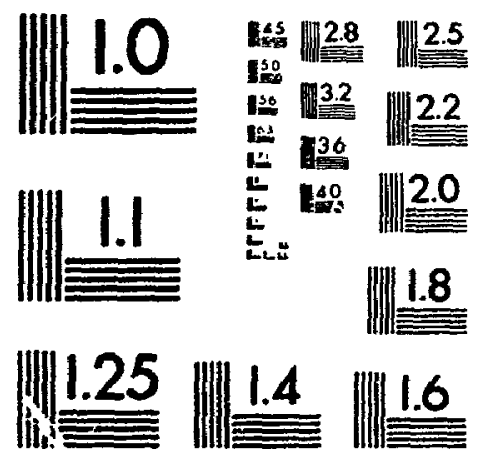

PRECISIONSM RESOLUTION TARGETS 
Acquisitions and

Bibliographic Services Branch

395 Wellington Street

Onawa, Ontarı

KIA ON4
Direction des acquisitions et des senices bibliographiques

395, rue Wellington

Ottawa (Ontario)

NOTICE

The quality of this microform is heavily dependent upon the quality of the original thesis submitted for microfilming. Every effort has been made to ensure the highest quality of reproduction possible.

If pages are missing, contact the university which granted the degree.

Some pages may have indistinct print especially if the original pages were typed with a poor typewriter ribbon or if the university sent us an inferior photocopy.
La qualité de cette microforme dépend grandement de la qualité de la thèse soumise au microfilmage. Neus avons tout fait pour assurer une qualité supérieure de reproduction.

S'il manque des pages, veuillez communiquer avec l'université qui a conféré le grade.

La qualité d'impression de certaines pages peut laisser à désirer, surtout si les pages originales ont été dactylographiées à l'aide d'un ruban usé ou si l'université nous a fait parvenir une photocopie de qualité inférieure.

La reproduction, même partielle, de cette microforme est soumise à la Loi canadienne sur le droit d'auteur, SRC 1970, c. C-30, et ses amendements subséquents.
Reproduction in full or in part of this microform is governed by the Canadian Copyright Act, R.S.C. iḡó, c. C-30, and subsequent amendments.

AVIS 


\title{
Training and Design of VLSI Neural Networks
}

\author{
by \\ George Chamberlain, B.Sc. \\ A thesis submitted to \\ the Faculty of Graduate Studies and Research \\ in partial fulfillment of \\ the requirements for the degree of
}

Master of Engineering

Ottawa-Carleton Institute for Electrical Engineering

Faculty of Engineering

Department of Electronics

Carleton University, Canada

October 25, 1994.

(c) George Chamberlain, 1994. 
National Library

of Canada

Acquisitions and Bibliographic Services Branch

395 Wellington Street Ontawa, Ontario

KIA ON4
Bibliotheque nationale

du Canada

Direction des acquisitions ef des services bibliographiques

395, no Wellingtion

Otlawa (Ontano)

Your hile votre retorence

Our the Nolre restarence

THE AUTHOR HAS GRANTED AN IRREVOCABLE NON-EXCLUSIVE LICENCE ALLOWING THE NATIONAL LIBRARY OF CANADA TO REPRODUCE, LOAN, DISTRIBUTE OR SELL COPIES OF HIS/FER THESIS BY ANY MEANS AND IN ANY FORM OR FORMAT, MAKING THIS . HESIS AVAILABLE TO INTERESTED PERSONS.
L'AUTEUR A ACCORDE UNE LICENCE IRREVOCABLE ET NON EXCLUSIVE PERMETTANT A LA BIBLIOTHEQUE NATIONALE DU CANADA DE REPRODUIRE, PRETER, DISTRIBUER OU VENDRE DES COPIES DE SA THESE DE QUELQUE MANIERE ET SOUS QUELQUE FORME QUE CE SOIT POUR METTRE DES EXEMPLAIRES DE CETTE THESE A LA DISPOSITION DES PERSONNE INTERESSEES.
THE AUTHOR RETAINS OWNERSHIP

OF THE COPYRIGHT' IN HIS/HER THESIS. NEITHER THE THESIS NOR SUBSTANTIAL EXTRACTS FROM IT MAY BE PRINTED OR OTHERWISE REPRODUCED WITHOUT HIS/HER PERMISSION.
LAUTEUR CONSERVE LA PROPRIETE DU DROIT D'AUTEUR QUI PROTEGE SA THESE. NI LA THESE NI DES EXTRAITS SUBSTANTIELS DE CELLECI NE DOIVENT ETRE IMPRIMES OU AUTREMENT REPRODUITS SANS SON AUTORISATION. 
Name George Chamberyan

Dissertation Abstracts international is arranged sy brood, general subject categories. Please select the one subject which most nearly describes the content of your dissertation. Enter the corresponding four-digit code in the spaces provided.

ELECTRONICS ENGINEERING SUBJECT IERM
$015[4] 4$ U.M.I SUASECT CODE

\section{Subject Cafegories}

\section{THI HUMANITIES AND SOCIAL SCIENCES}

\section{COMMUNiCATIOHS AND THE ARTS}

Architacture ... . ......0729

Ant History . . . . . . . 0377

Cinema $. . ., \ldots \ldots \ldots \ldots, 0900$

information Science

Journalism........ .0391

Library Science . . ...0399

Mass Communications ...... .0708

Music Communication $\quad . . . . .0459$

\section{EDUCation \\ General}

Aduli and Continuing

Agriculirural

Bilingual and Mulficultural “ .0282

Businass
Community College

Curriculum and Instruction ... 0727

Early Childhood. .. . .....0518

Elementary. $\quad \cdots \quad .0524$

Fuidance ond Counseling .... $05: 9$

Guidance ond Counseling .... 0680

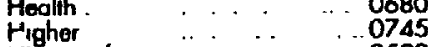

\begin{tabular}{lll} 
History of & .0520 \\
Home Economics & $\cdots$ & 0278 \\
\hline
\end{tabular}

Industriol 0521

language and Literature .. ... 0279

Manhemalics . ..... 0280

$\begin{array}{llll}\text { Philosophy of } & \ldots . . & 0 & 0998 \\ \text { Physical } & . . & . . & 0523\end{array}$

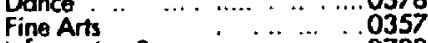

Administration

Bilingual and Multicultural $\quad .0688$

$\begin{array}{llll}\text { Music } & & 0522 \\ \text { Philosophy of } \quad \ldots . . & 0998\end{array}$

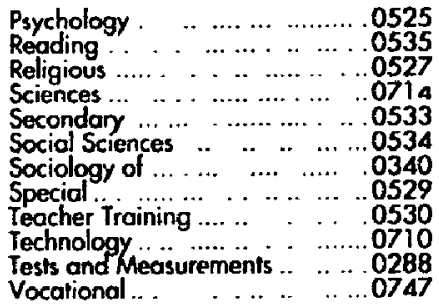

\section{LNGUAGE, LITERATURE AND}

UNGUISTICS

Language

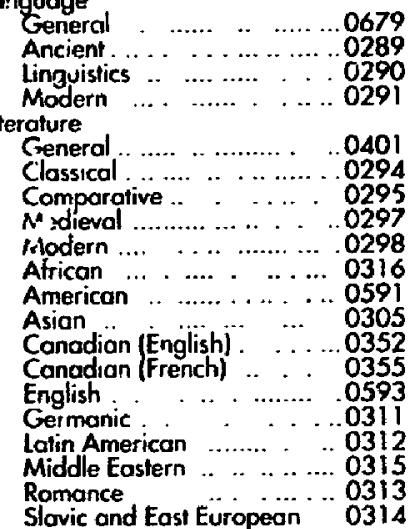

PHIIOSOPHY, RELIGION AND

\section{THEOLCOY}

Philosophy. . .... .............. 0422

Religion

General ............. 0318

Biblical Studies . .............. 0321

Clergy ... ... . ...............0319

Philosophy of ... . ............. 0322

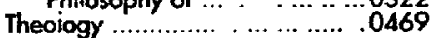

\section{SOCIAL SCIENCES}

Americon Studies . ......... . . ...0323

Anthropology

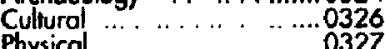

usiness Administration,

General ................... 0310

Accounting ................. .0272

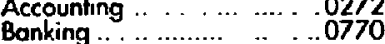

Management ....................... 0454

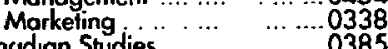

Canudian Studie

Economics

General ...................0501

Agricultural . $\quad \ldots \ldots \ldots \ldots . . .0503$

Commerce-Business ........... .0505

Finance .. $\quad . . \ldots \ldots \ldots . . . .508$

$\begin{array}{llll}\text { History. } & \ldots \ldots \ldots \ldots & \ldots . . & 0509 \\ \text { labor } & \ldots \ldots \ldots \ldots & & 0510\end{array}$

Labor

Folklore

Geography

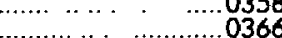

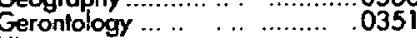

History

General ...

.0578
Archaeology $\ldots \ldots \ldots \ldots .0324$

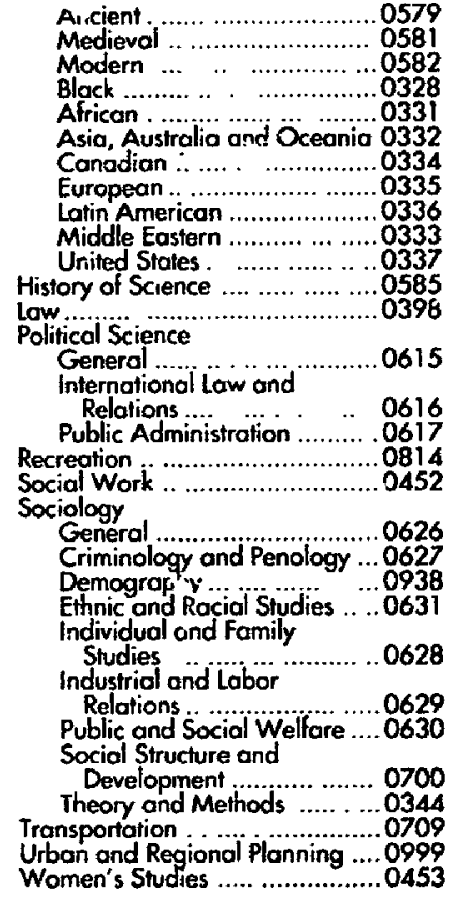

THE SCIENCES AND ENGINEERINO

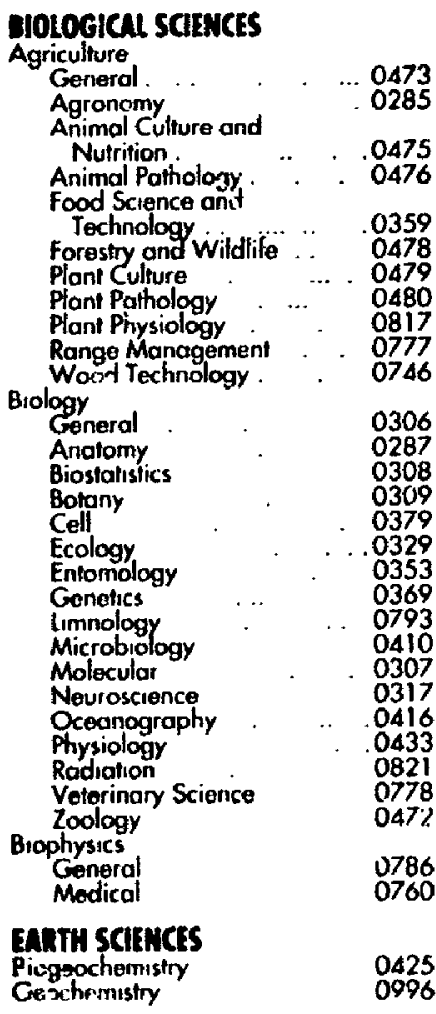

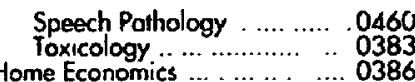

\section{PHYSICAL SCIENCES}

Pure Sciences

Chemistry

General ............. 0485

Agricultural ......... 0749

$\begin{array}{llll}\text { Analytical .... . . } & \ldots . . & \ldots .0486 \\ \text { Biochemistry } & & \ldots & . . .0487\end{array}$

Inotenic

Nuclear .... .............0738

Organic. ................... 0490

Prganic. ...

Physical .................0494

Polymer . . ...............0495

Environmental Sciences . . . .0768

Health Sciences

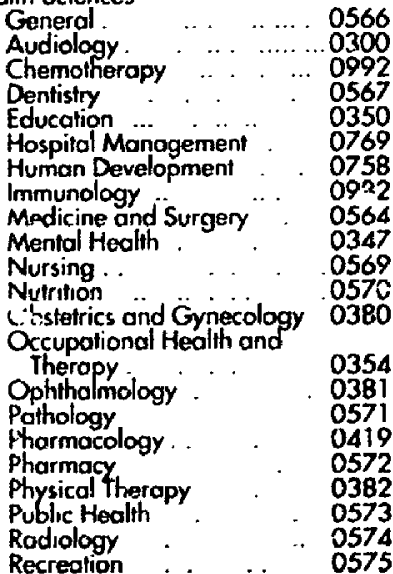

Astrophysics.................0606

Atomic .... . ...... 0748

Nueless ............ . ......0610
Mathematies . .... .......... 0405

Physics

General ....... 0605

Acoustics ....................... 0986

Astronomy and

Atmospheric Science ...........0608

Electronics and Electricity ...0607

High Energy. ........ 0798

Fluid and Plasma .. . . .... 0759

Molecular ................. 0609

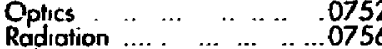

Solic' State $\ldots . . . . . . . . .0611$

Statistics ... . . . ................ 0463

Applied Sciences

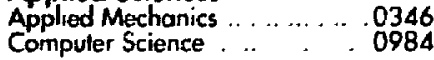

Engineering

Aerospoce

Agricultural.

Automotive

Biomedical

Chemical ..................

Electronics and Electrical ..... 0544

Hydraulic

Industrio

Marine

(1).....

Materials Science .. .............0794

Mechanical. ... ...............0548

Metallurgy . ..... . ... .... 0743

Mining ....................... 055

Nuclear ........................ 0552

Packaging ...................... 0549

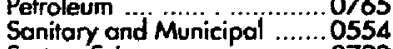

System Science .................. 0790

Geolechnology ...................0428

Plastics Technology ................... 0795

Textile Technology .................... 0994

\section{PSYCHOLOOY}

General

Behavioral .........................062

Clinicol .............................0622

Developmental ......................0620

Experimental. . ...................0623

Industrial .... ...................0624

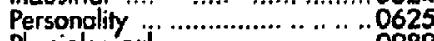

Physiolagical .......................0989

Psychobrology .......................

Sorid .................. 0451 
The undersigned recommend to the Faculty of Graduate Studies and Research acceptance of the thesis

\section{"Training and Design of VLSI Neural Networks"}

submitted by

George Chamberlain, B.Sc.

in partial fulfillment of the requirements

for the degree of Master of Engineering

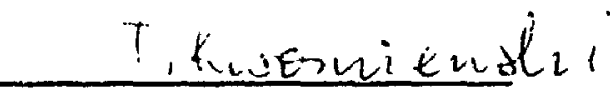

Thesis Supervisor

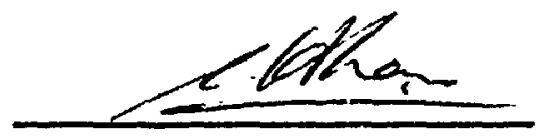

Chairman,

Department of Electronics

Carleton University 


\section{Abstract}

An examination of different hardware technology options for the implementation of artificial neural networks (ANNs) finds that CMOS integrated circuit technology using analog circuit techniques is best suited for the development of large ANNs. Numerous implementation schemes from the literature are examined and, based on the results, an new architecture is selected which incorporates floating gate differential pair basedsynapses, a current-to-voltage converter-based soma, and a transconductance amplifierbased activation function.

Since there is close coupling between the ANN hardware and the training algorithm, both the backpropagation and the cascade-correlation training algorithms are examined for their suitability to the training of VLSI-implemented ANNs. It is found that the dynamically-generated network architecture and reduced precision requirements of cascade-correlation render it better suited to the training of VLSI-implemented ANNs, though still deficient in the modelling of non-ideal synapses. A modified cascad:correlation algorithm is proposed, and its ability to overcome this deficiency is shown. 


\section{Acknowledgments}

I would like to express my sincere thanks to my thesis supervisor, Dr. T. Kwasniewski, for his continued faith, prodding, and support during the research and writing of this thesis.

I am also grateful to Wendy Doyle, and my other friends and co-workers, who, over the course of this thesis, disp!ayed remarkable patience.

Finally, my parents deserve special thanks for their unfailing confidence. 


\section{Table of Contents}

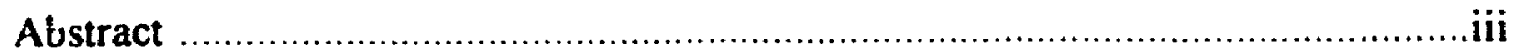

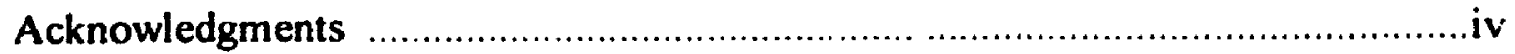

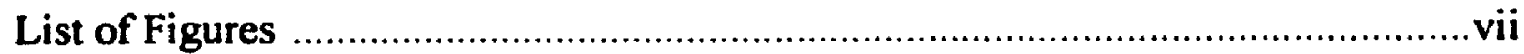

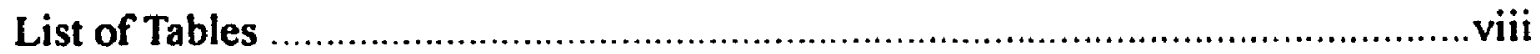

List of Terms, Acronyms, and Symbols ..................................................

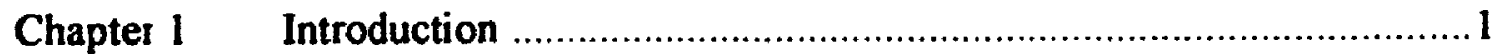

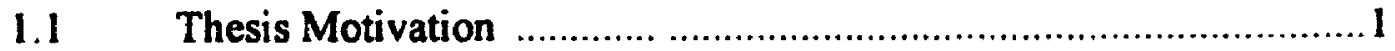

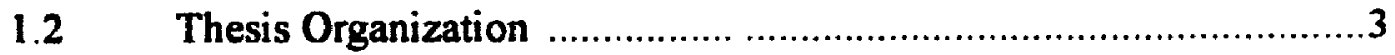

Chapter 2 Introduction to Neural Networks .......................................... 5

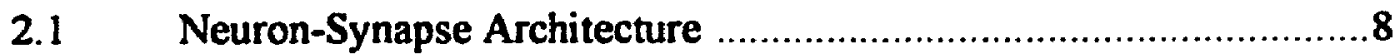

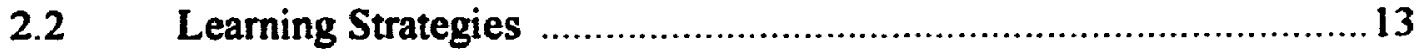

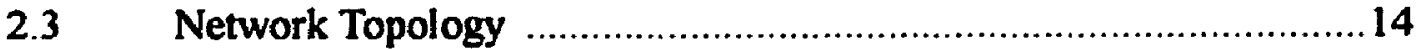

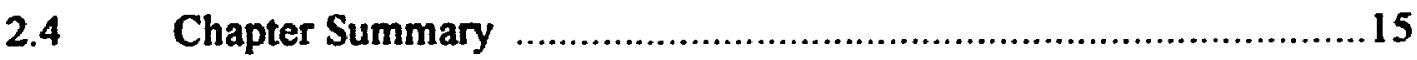

Chapter $3 \quad$ Neural Network Training Algorithms ..............................18

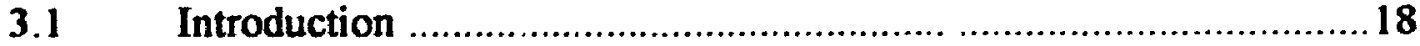

3.2 The Delta Rule-Based Training Algorithm ........................... 18

3.3 Backpropagation Training Algorithm ................................20

3.3.1 Learning Using the Backpropagation Training Algorithm ....21

3.4 Deficiencies of the Backpropagation Training Algorithm .............24

3.5 Cascade-Correlation Training Algorithm ............................27

3.5.1 A Comparison of Backpropagation and the CascadeCorrelation Training Algorithms .................................28

3.5.2 Learning Using the Cascade-Correlation Training Algorithm .............................................................. 1

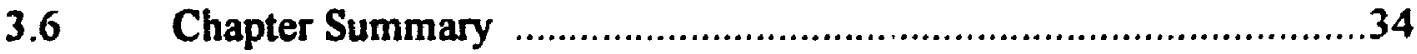

Chapter $4 \quad$ Neural Network Implementations ........................................36

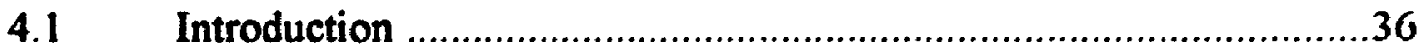

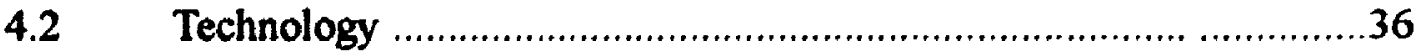

4.3 CMOS Design Criteria .............................................. 40 


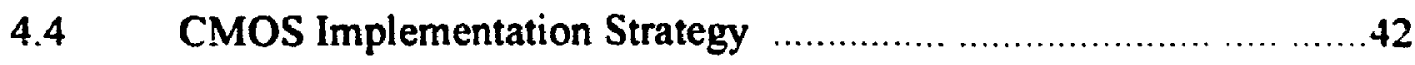

4.5 Weight Storage Considerations …….........................................46

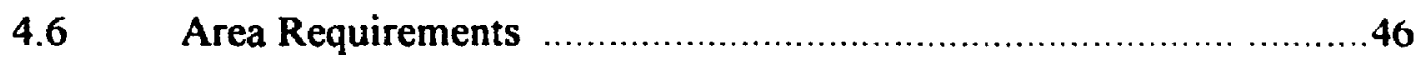

4.6.1 Synaptic Storage Elements and Numerical Precision............47

4.6.2 Synaptic Restrictions Imposed by Application

Requirements ...............................................................49

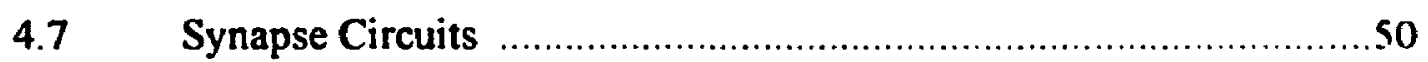

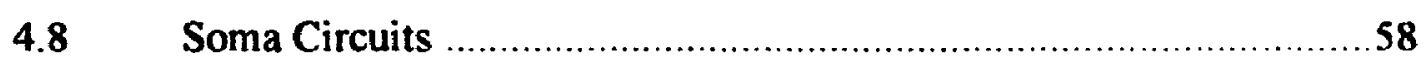

4.9 Analog Implementations of Activation Function ......................60

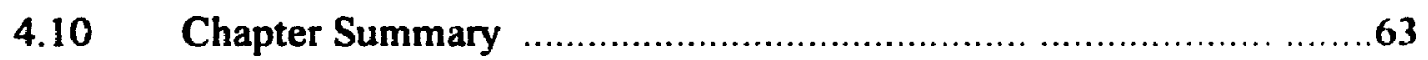

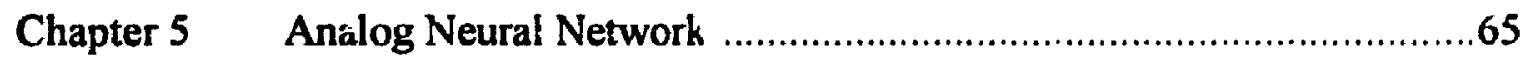

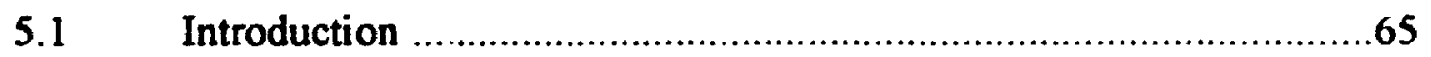

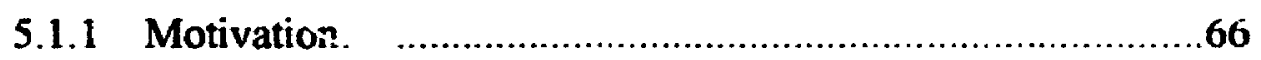

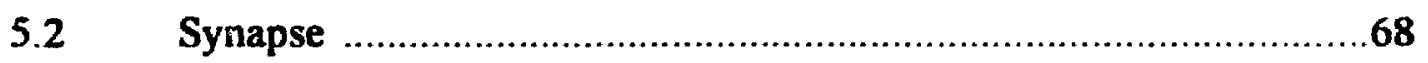

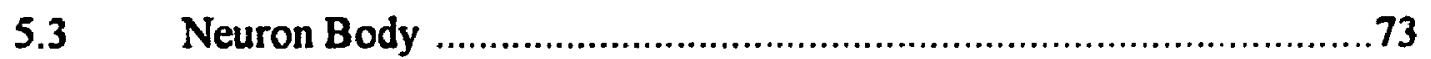

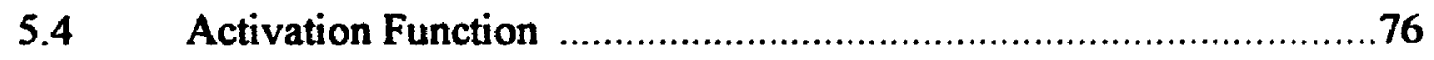

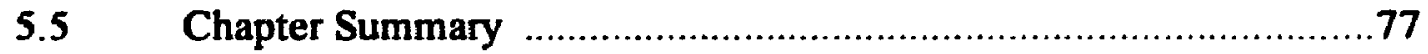

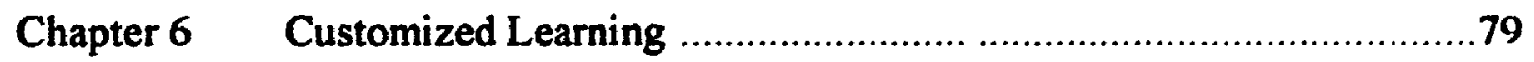

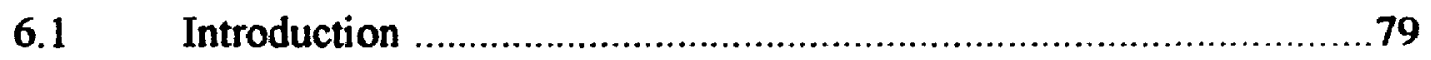

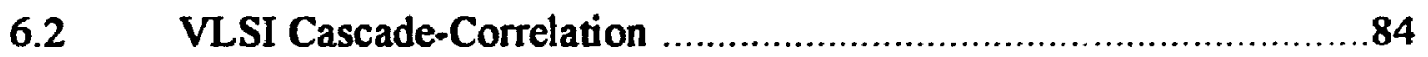

6.3 Justification of VLSI Cascade-Correlation ...............................88

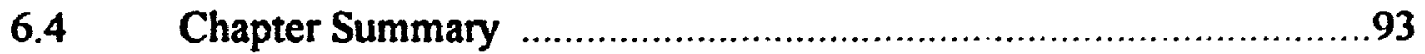

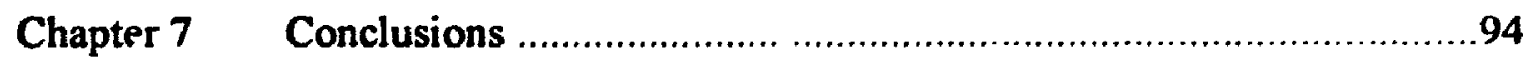

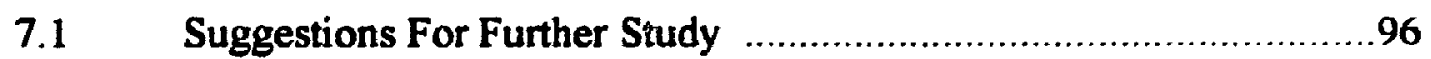

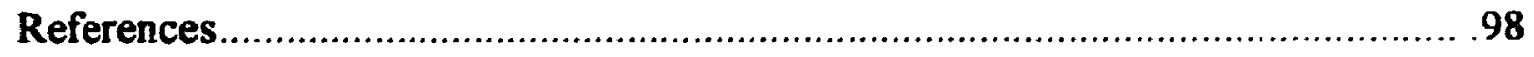




\section{List of Figures}

FIGURE 1 Perceptron Single-Neuron Model ............................................ 9

FIGURE 2 Single-Layer Perceptron Network ........................................ 10

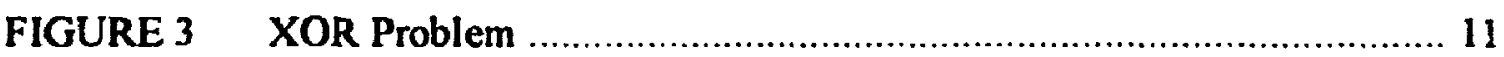

FIGURE 4 Feed-Forward Multi-Layer Perceptron ................................... 12

FIGURE S Activation Functions....................................................... 14

FIGURE 6 Fully Connected Artificial Neural Network .............................. 16

FIGURE 7 Cascade-Correlation Neural Network ...................................... 17

FIGURE 8 Backpropagaiion Training Procedure ................................ 23

FIGURE 9 Cascade-Correlation Training Procedure................................. 34

FIGURE 10 Neural Network Implementation Technology ........................... 37

FIGURE 11 Local Layout Style for Large Neural Network........................... 45

FIGURE 12 Op-Amp-Based Inner Product Circuit ...................................... 51

FIGURE 13 Programmable Capacitor ................................................. 52

FIGURE 14 Switched-Capacitor Resistors ......................................... 53

FIGURE 15 MOSFET Resistor-Based Inner Product Circuit ........................... 55

FIGURE 16 Transconductance Amplifier-Based Inner Product Circuit.............. 56

FIGURE 17 Reduced-Transistor-Count Synapse. .................................... 57

FIGURE 18 Linear IV Converter ............................................................. 59

FIGURE 19 Current Thresholder ......................................................60

FIGURE 20 Transconductance Amplifier-Based Activation Function..............61

FIGURE 21 CMOS Inverter-Based Activation Function .................................62

FIGURE 22 Neuron-Synapse Structure ................................................65

FIGURE 23 Floating-Gate, Differential Pair-Based Synapse ......................68

FIGURE 24 Simpler Synapse Configuration .........................................69

FIGURE 25 Synapse Simulation..................................................... 72

FIGURE 26 Linear Current-to-Voltage Converter ................................. 73

FIGURE 27 Linear Current-to-Voltage Simulation .................................. 75

FIGURE 28 Transconductance Amplifier Activation Function.................... 76

FIGURE 29 Activation Function Simulation Result................................. 78

FIGURE 30 Integrated and Linear Synapse Responses................................ 91

FIGURE 31 Neuron Output Assuming Both Linear and Non-Linear Synapses.... 92 


\section{List of Tables}

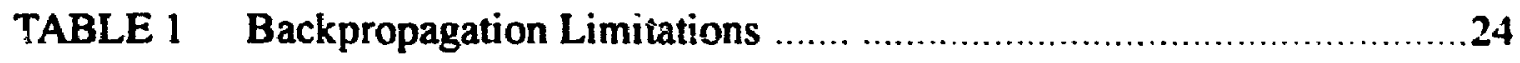

TABLE 2 Comparison of Fixed Hardware Technologies ................................

TABLE 3 L: . gital vs. Analog Implementation Strategies ..............................40

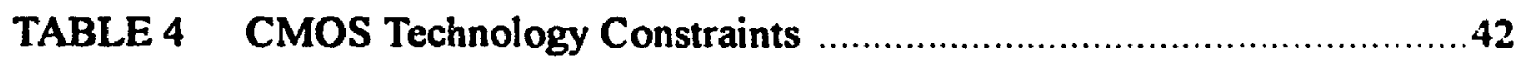




\section{List of Terms, Acronyms, ana Symbols}

Neural Network Specific Terminology

ANN Artificial Neural Network

Backpropagation Widely used neural network training algorithm

Cascade-

Correlation Supervised neural network training algorithm that dynamically creates the network during training

Deita Rule Neural network training algorithm used when incoming weights to the hidden layers do not require training

Network Depth The number of hidden layers of in a network

Epoch

One weight update phase during training where the complete training set is applied to the network

Hidden Layer A layer of neural units connected neither to the inputs of the network nor to the outputs of the network

MLP Multi-Layered Perceptron

Perceptron Rosenblatt neuron with sgn() function activation

Quickprop Second-order minimizaticn/maximization algorithm used by cascade-correlation

Network

Topology

SLP

Soma

Supervised

Learning

Refers to the orgarization of the hidden layers within the a network Single Layer Perceptron Network

Neuron body that accomplishes summation of weighted inputs and the activation function

A class of training algorithms for training neural networks where both the inputs and associated outputs are required

Synapse

Two-Spiral

Problem

Unsupervised

Learning

Neural element that accomplishes weight storage and modulation of the input signal

Weight

A benchmarking test for neural network training algorithm:s involving the distinguishing of two spirals

A class of training algorithms for training neural networks for which a-prion knowledge of the output pattern is not required Controls the strength of input connection 
Yield The ratio of the good devices on a wafer to all the devices on a wafer

Integrated Circuit Specific Terminology

BiCMOS Bipolai Complementary Metal Oxide Semiconductor

CCD

Charge-Coupled Device

CMOS

Comp!ementary Metal Oxide Semiconductor

DRAM

Dynamic Random Access Memory

EEPROM

Eiectrically Erasable Programmable Read Only Memory

GaAs

Gallium Arsenide

IC

Integrated Circuit

MNOS

Metal Nitride Oxide Semiconductor

MOSFET

Metal Oxide Semiconductor Field Effect Transistor

NMOS

N-channel MOSFET

PGA

Pin Grid Array

PMOS

P-channel MOSFET

SPICE

Device-level circuit simulation tool

SRAM

Static Random Access Memory

VLSI

Very Large Scale Integration

General Terminology

LMS

$\operatorname{sgn}(\mathbf{k})$

SIMD

\section{Symbols}

$d_{p j}$

$E_{o}$

$E_{\text {op }}$

$E_{p j}$

$f$

\section{Least Mean Squared}

Function such that output is 1 if $k>0,-1$ otherwise

Single Instruction Multiple Data parallel computer architecture

Target output value for neuron $j$ when presented with pattern $p$ The LMS error averaged over all input patterns at output node 1 . The LMS error at a given output node, $o$, for pattern $p$. LMS error between desired and actual network outputs for test pattern $p$, output neuron $j$

Neuron activation function 
Non-linear summation performed by curient thresholder

Non-linear multiplication performed by synapse

Current

$I_{D D}$

Drain saturation current at $V_{G S}=V_{D D}$

$N_{o}$

Number of output units

$\boldsymbol{o}$

Output unit

$P_{j}$

Neural network input pattern to element $j$

Precision function - quantizes $\boldsymbol{x}$ to $\boldsymbol{n}$ bits of precision

$\boldsymbol{s}_{\boldsymbol{i}}$

Input to the neuron activation function - a weighted sum of the input signals

V

$V_{D o}$

$w_{i j}$

$\Delta w_{t-1}$

$y_{p j}$

$\alpha$

$\varepsilon$

$\lambda$

$\mu$

Voltage

Drain saturation voltage of at $V_{D S}=V_{D D}$

Weight connecting output of neuron $i$ to input of neuron $j$

Value of the weight change at the previous epoch

Output of neuron $j$ when presented with pattern $p$

Velocity saturation index

Gracient-descent term used by quickprop to start the training procedure

Channel length modulation factor

Maximum growth factor term used by quickprop to limit the size of weight updates.

Neuron threshold

Learning rate 


\section{Chapter 1. Introduction}

\subsection{Thesis Motivation}

This work addresses two major issues that factor into the design of successful ANNs - the selection of an implementation technology (Chapter 4), and the seleciion of an appropriate learning algorithm (Chapter 3).

Implementation technology options include software simulations, digital integrated circuit (IC) teinnology, and analog IC technology. Software simulations are the most common. However, networks modelled in software suffer from the same efficiency failings as standard computing; sequential computation. Aithough parallel computing can speed-up execution significantly, neural architectures and training algorithms are not efficiently mapped to standard parallel hardware due to the extremely high communication requirements between distant processors.

Digital IC implementations are relatively straightforward to design and model, since they are designed at the logic level rather than the transistor level. Unfortunately, the straightforward design is complicated by the large amount of area required to implement each function. As the amount of circuitry used to implement a function increases, so do both the cost and the probability of manufacturing defects, making the network impractical to build. Further, although digital designs can, in theory, support arbitrary numerical precision, this cannot be realized in practical implementations. As a consequence, digital circuitry is limited to the implementation of small neural networks. 
Analog IC implementations, by contrast, can be quite compact. Notwithstanding, analog implementations still have shortcomings of their own. Due to their compact size, they often produce approximations, rather than exact representations, of the functions they are meant to implement. Their dynamic range - which can be considered equivalent to the numerical precision in digital circuitry - is limited, which in turn limits the trainability and the usefulness of the network. Nonetheless, when considered against the area requirements of digital implementations, the shortcomings of analog implementations are relatively minor.

This work justifies an analog Complementary Metal Oxide Semiconductor (CMOS) IC implementation of an ANN with simple subcircuits - a differential pair using floating gate transistors, a linear current-to-voltage converter (seven transistors) and a differential amplifier (five transistors) - for the implementation of one synapse-neuron pair (Chapter 4).

Using these simple subcircuits, the challenge of designir:g a successful CMOS analog ANN is use the training algorithm to compensate for both the limited dynamic range and the non-ideal approximations of functions displayed by analog circuitry.

Traditional training algorithms, such as backpropagation and cascade-correlation, do not incorporate these features. This work thus proposes a new algorithm (Chapter 6), the VLSI cascade-correlation algorithm, that compensates for the limitations of analog circuity, and allows closer matching of computer simulated device behavior to the actual device behavior without the need for SPICE-like device simulation during training. 


\subsection{Thesis Organization}

The remainder of this thesis is composed of six chapters.

Chapter 2 introduces AINN design independent of the implementation media. The basic principles of neural networks, the motivation for using neural networks, as well as neural network architectures, are also presented. An introduction to the classes of leaming algorithms used to train neural networks is also included.

Chapter 3 is an in-depth discussion of supervised learning algorithms, starting with the delta rule training function, followed by backpropagation, and finally cascade-correlation. Backpropagation is the most widely used algorithm for training ANNs. Some of the drawbacks of backpropagation are presented, along with a comparison of backpropagation and cascade-correlation. The comparison shows that cascade-correlation training algorithm is more suited to the training of VLSI-implemented neural networks than the backpropagation algorithm.

Chapter 4 presents a feasibility study that compares the suitability of various technologies to the implementation of ANNs. CMOS is found to be the most suitable technology. Since many different design strategies are available using CMOS, the study first compares general analog and digital design techniques. Analog design techniques are found to be more favorable for the creation of large neural networks. At this point, a number of different analog techniques that have been presented in the literature are examined for their suitability to the implementation of the subcircuits reguired to create a neural network. Finally, an architecture that differs from any presented in the literature is defined. 
This architecture is based on a floating gate differential pair for the synapse, a linear current-to-voltage converter for the soma (neuron body), and a transconductance amplifier for the activation function.

Since the hardware used to implement an ANN cannot be considered independently of the learning algorithm, Chapter 5 provides a more in-depth analysis of the new architecture presented in Chapter 4, with the express purpose of extracting simple models for use in the modified cascade-correlation training algorithm developed in Chapter 6 . This algorithm is shown to provide better correlation between the actual behavior of the device (as illustrated through SPICE simulations) and the learning algorithm than the standard cascade-correlation algorithm.

Finally, Chapter 7 presents the conclusions of this research and provides some suggestions for tuture work. 


\section{Chapter 2. Introduction to Neural Networks}

ANNs are adaptive structures constructed using many interconnected active components. called neurons. Each neuron is connected to many other neurons through interconnections called synapses. These synapses moderate the input signal through the programming of a weight value, thus controlling the level of influence, or contribution, each input to a neuron has on that neuron's output response. The process of defining the weight values in a network is called training. It is the training process that distinguishes an ANN designed for one application from another. Although ANNs can be configured with different numbers of inputs, outputs, and hidden neurons (neurons attached neither to an input nor an output), these can only be applied to solve real-world problems after their weight values have been defined through the training process. A network that has not been trained is similar to a palette and a blank canvas without a painting.

The training process proceeds by applying a set of input patterns to the ANN and recording the response. If the response is not as expected, based on the difference between the desired response and the actual response, the network weight values are adjusted. Once the actual response is similar to the desired response for all the input patterns, the network is considered trained. Since the desired response of the network is used to train the network, this type of leaming is called supervised !earning ${ }^{[2]}$. There is a second type of learning, called unsupervised learning ${ }^{\{2\}}$, which trains the network by looking for similarities in training set patterns, and learning to recognize them without the aid of a teacher. 
One of the advantages of ANNs over traditional problem-solving techniques is that the networks can solve problems of the same type as the ones used to train the network, but that the network has never before seen. For instance, if a network is trained so as to distinguish the handwritten word "hello", once training has been successfully completed, the network should be able to recognize any handwritten instance of the word "hello", even if it wasn't contained in the training set. The network has the ability to generalize on what it has been taught without requiring a-priori knowledge of the transfer function required to solve the problem.

Although the network is composed of very simple elements - the synapse simply performs a multiplication between the input signal and a stored programmable weight value, and the neuron accomplishes a summation of all the "weighted" inputs and performs a simple activation function (discussed below) - it can solve very complex problems. This can be achieved since many synapse connections are available in the network, allowing for a high degree of programmability.

Each neuron in the neural network performs the same function,

$$
y_{1}=f\left(\sum_{z=1}^{N}\left(w_{1 j} p_{z}+\theta_{j}\right)\right)
$$

where $f$ is the activation function, $p_{i}$ is the input signal from neuron $i, w_{i j}$ is the weight, or synapse value, connecting the output of neuron $i$ to the input of neuron $j$, and $y_{j}$ is the neuron output. The neural network contains $N$ inputs and $M$ outputs ( $j$ runs from 1 to $M$ ). The neuron threshold is represented by $\theta$. 
Further, although the speed of each neuron may be low, the high degree of parallelism inherent in the network allows many types of inoblems to be solved quickly and efficiently.

An ANN can be viewed as a network of simple weighted summation nodes, each followed by a non-linear activation function. Each neuron input is modified by a weight before the summation. This modification takes place in the synapse. The summation operation takes place in the neuron body, or soma. The activation function modifies the summation in either a linear or a non-linear manner (Section 2.1).

Recent neural network research can be broadly separated into two categories. The first focuses on the development of detailed models of the brain to allow the design of sensors whose function is based on brain function. ${ }^{[3]}$ Numerous examples of this type of research. including an optical motion sensor, a silicon retina, and an electronic cochlea, have been presented by Mead. ${ }^{[4]}$ Mead and Mahowald's silicon retina, for example, is modeled on the distal portion of the vertebrate retina. Although quantitative results are not presented, they report that their silicon retina behaved quite favorably compared to biological systems. Neural models rigorously based on biological models not only aid in the development of new sensors, as illustrated above, but can provide insight into the functioning of the brain. If the model displays similar characteristics to the biological system, the model can be used as predictive tool.

The second area of ANN research - which is also the focus of this thesis - is the generation of networks that can be used to solve practical computational-type problems Research of this type relies on primitive neuron and synapse models to construct a large 
network composed of simple components, and capable of solving complicated problems when trained. Such networks are normally trained using the training algorithm that yields the best results. Since biologically plausible networks are not a requirement, different learning algorithms can be evaluated for their suitability to the application. Most commonly, the backpropagation learning algorithm which is introduced in Section 2.2 and discussed in detail in Chapter 3, is employed.

\subsection{Neuron-Synapse Architecture}

The ANNs being studied in this thesis are historically based on the single layer perceptron

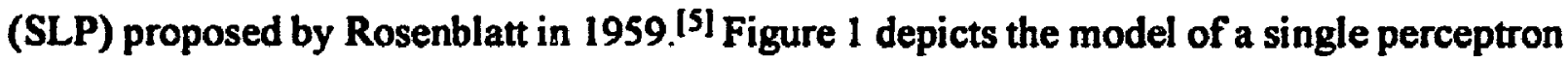
unit. A network is generated by arranging the perceptrons into two layers, input and output layers, with the output of each perceptron in the input layer serving as an input to every perceptron in the output layer, as shown in Figure 2. A weight (synapse) moderates the connection strength. Typically, no connections exist between perceptrons on the same layer.

Each perceptron in the output layer - composed of the input synapses, the summing, and the activation functions - perform the operation defined by Equation 1. The input layer serves only to distribute the signals to the output perceptrons.

The weight value is stored in the synapse where the $w_{i j} p_{i}$ inner-product operation is calculated. The output function for the perceptron is usually a linear activation function or 
the $s g n$ function:

$$
f(i)=i \quad \text { or } \quad f(i)=\operatorname{sgn}(i)=\left\{\begin{array}{ll}
+1 & , i>0 \\
-1 & , i \leq 0
\end{array} .\right.
$$

The neuron transfer function given by Equation 1 can be rewritten without the threshold term being explicitly noted. The threshold value can be expressed by the multiplication of

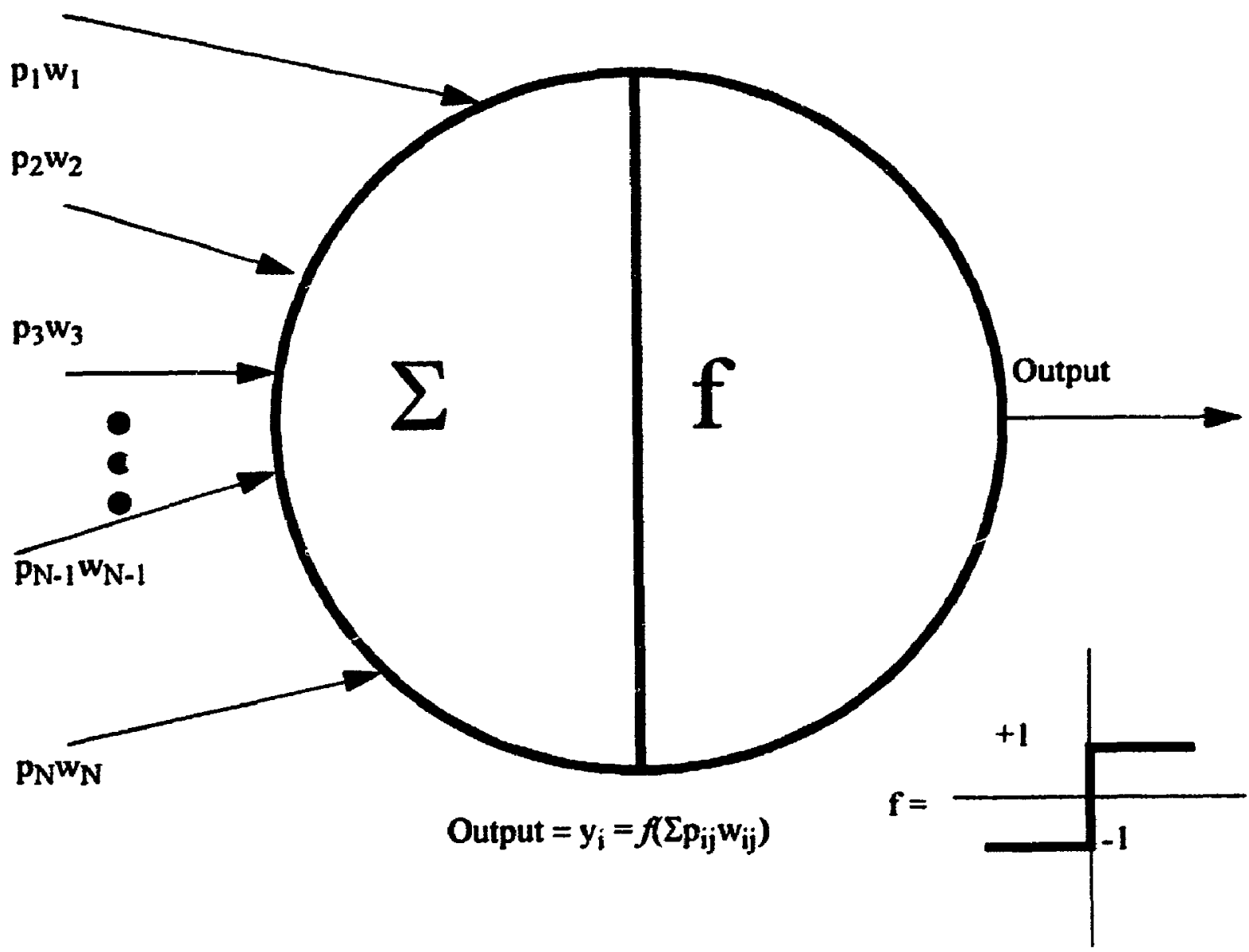

FIGURE 1 : Derceptron Single-Neuron Model

A weighted sum of the input signal $\left(p_{N}\right)$ is performed by the synepses and neuron. The activation function of the neuron () , in this example, accomplishes a binary threshold 
a fixed weight with a fixed input,

$$
\theta_{j} \equiv w_{0,} p_{0 j}
$$

thus reducing Equation I to:

$$
y_{1}=f\left(\sum_{i=0}^{N} w_{i j} p_{1}\right)
$$

The perceptron model can be applied to a wide variety of problems and was the subject of research through most of the 1960s. In 1969, however, Minsky and Papert published results clearly defining the limitations of perceptrons. ${ }^{[6]}$ Since the perceptron architecture of Figure 2 employs no hidden layers, only linearly separable problems could be solved.

Linearly separable problems - where the boundary between two regions, or data points,

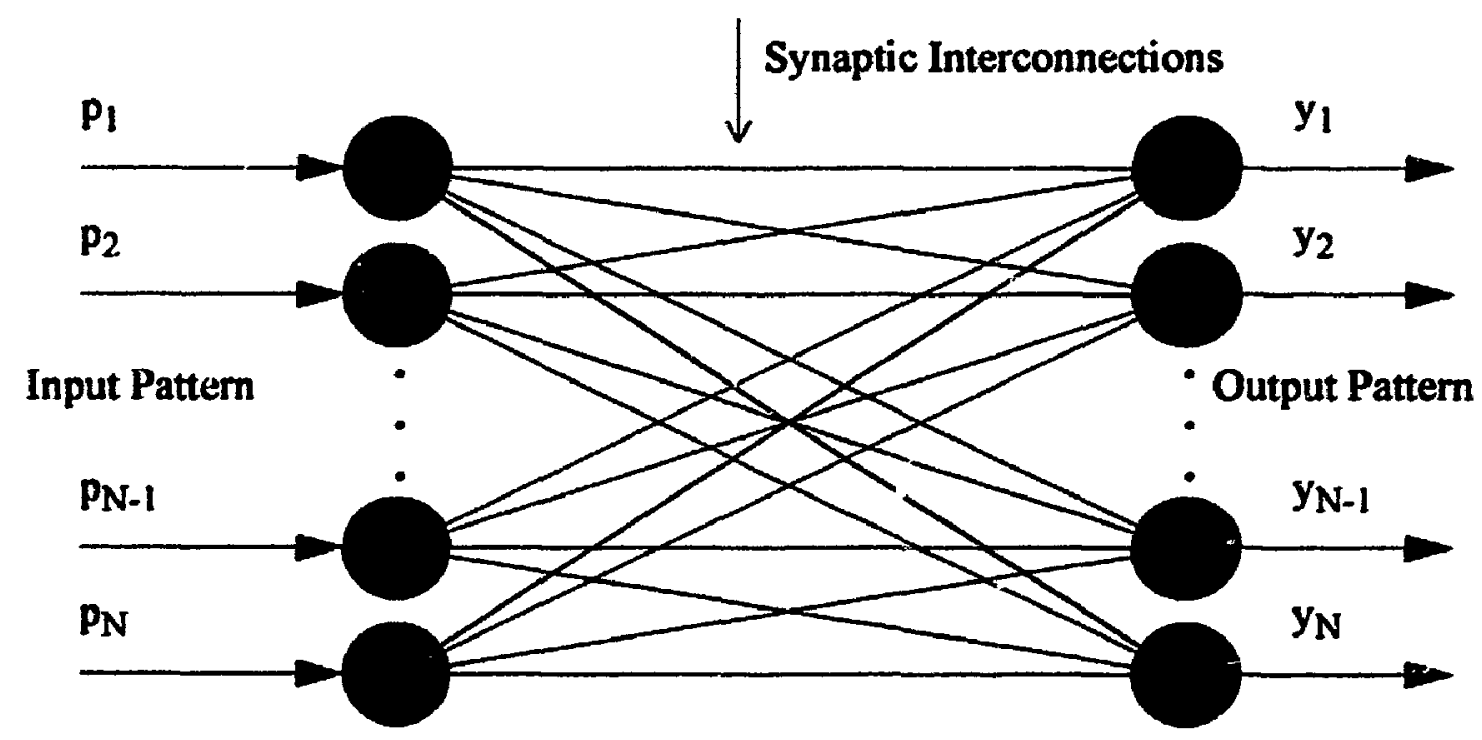

FICURE 2 : Single-Layer Perceptron Network

Only connections between the input and the output layers are allowed. There are no hidden neurons. The input neurons serve only to route the data to the output layer. 


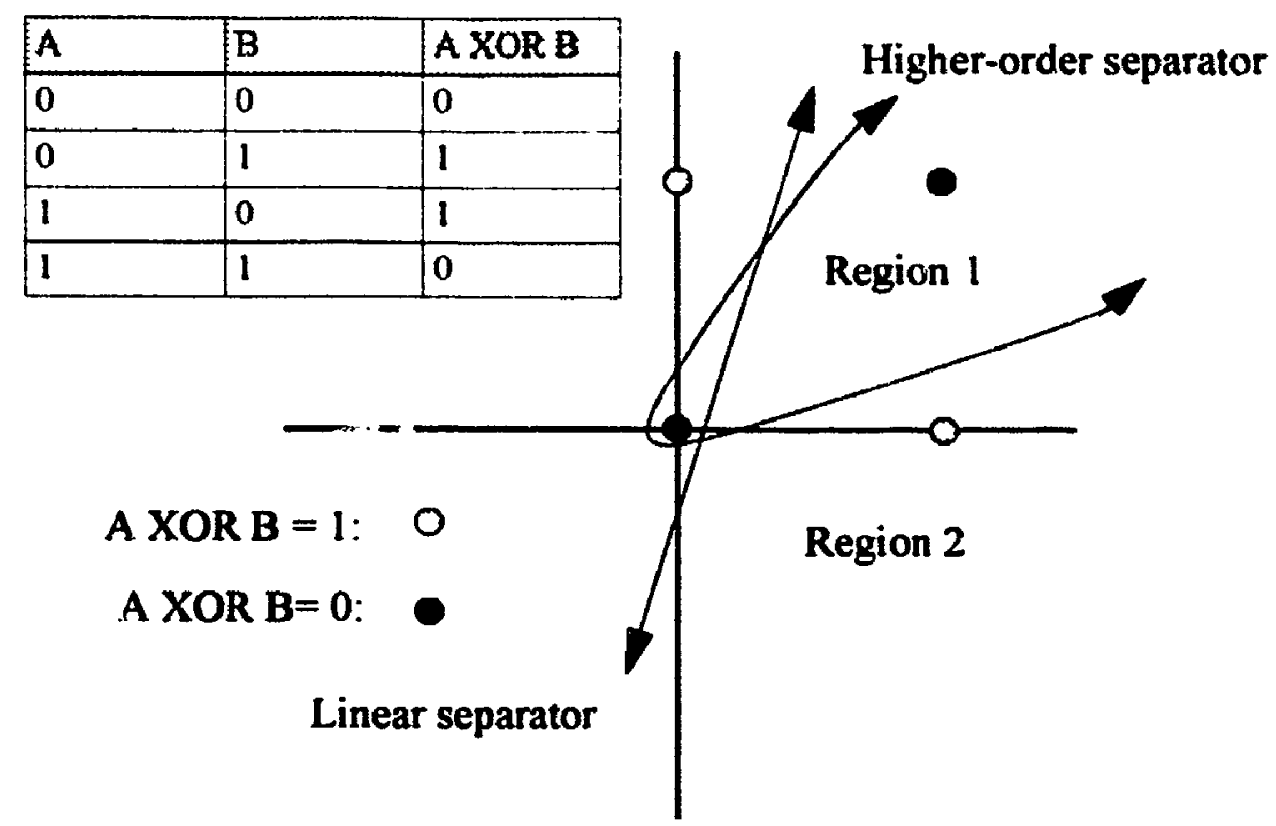

FIGUREE 3 : XOR Problem

The linear separator cannot define two regions such that the elements of $A$ are distinct from the elements of $B$ whereas the higher-order separator can.

must be separable using a straight line - are the most simple classification problems. The classic example of a simple problem that cannot be solved using the single layer perceptron is the XOR problem. As shown in Figure 3, to solve the XOR problem, the SLP must divide the plane into two distinct regions using a non-linear separator, illustrated in this case as a parabola. Since the data points cannot be distinguished by a linear separator, the problem cannot be solved using the SLP.

This result caused ANN research to stagnate for the next 15 years. Although it was known that a network comprised of non-linear activation units with hidden layers could solve arbitrary classification problems, no analytical training algorithm existed until backpropagation was discovered in 1986. Backpropagation is a neural network training algorithm that can train networks containing both non-linear activation functions and 


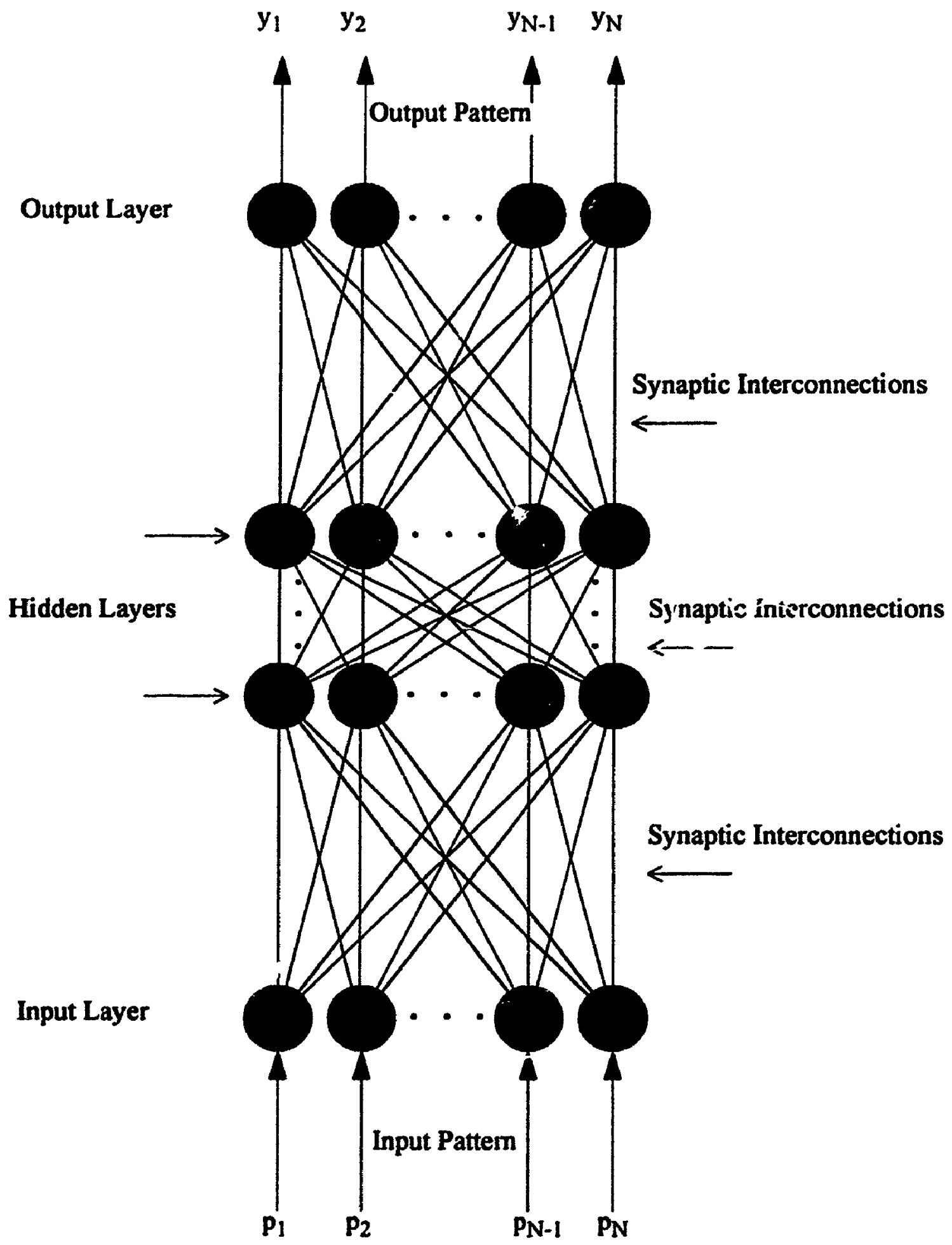

FIGURE 4 : Feed-Forward Multi-Layer Perceptron

The input layer neurons differ from the other neurons in that they only distribute the inputs to the neurons in the second layer. An arbitrary number of hidden layers can be present. Alinough the network illustrated above contains an equal number of units in each layer, an arbitrary number of neurons may be present in each layer. The number of input and output units are determined by the application. 
hidden units.

Backpropagation allowed the development of the multi-layar perceptron (MLP). Although backpropagation allows the training of arbitrary non-recurrent networks ${ }^{1}$, the layered feed-forward network shown in Figure 4 is the most usual architecture. The neuron used in backpropagation networks is quite similar to that of the perceptron, differing only in the activation function, which is usually given by

$$
f\left(p, w_{k j}\right)=\tanh \left(p, w_{k 1}\right) \quad \text { or } \quad f\left(p_{1} w_{k 1}\right)=\frac{1}{1+e^{-p_{0} w_{k}}}
$$

Typically, Equation 2 is not used as an activation function since it is not continuously differentiable. This causes problems for the backpropagation algorithm (Section 3.3.1). As a result, either the tanh() (hyperbolic tangent) or sigmoid functions presented in Equation 5 are normally used. As shown in Figure 5, they have similar characteristics to the sgn() function, but are continuously differentiable. The $\operatorname{tanh()~function~is~better~suited~}$ to VLSI architectures because it is simpler to implement than the sigmoid. The sigmoid is desirable because its derivative is easily calculable as the product of itself, times one minus the input value. The calculation of the derivative is reduced to a simple multiplication and subtraction.

\subsection{Learning Strategies}

In both supervised and unsupervised learning, ANNs learn by modifying the connection weights between neurons. Supervised learning requires that the network be provided with

1. To implement recurrent networks - networks with feedback - some modifications to backpropagation are necessary. 


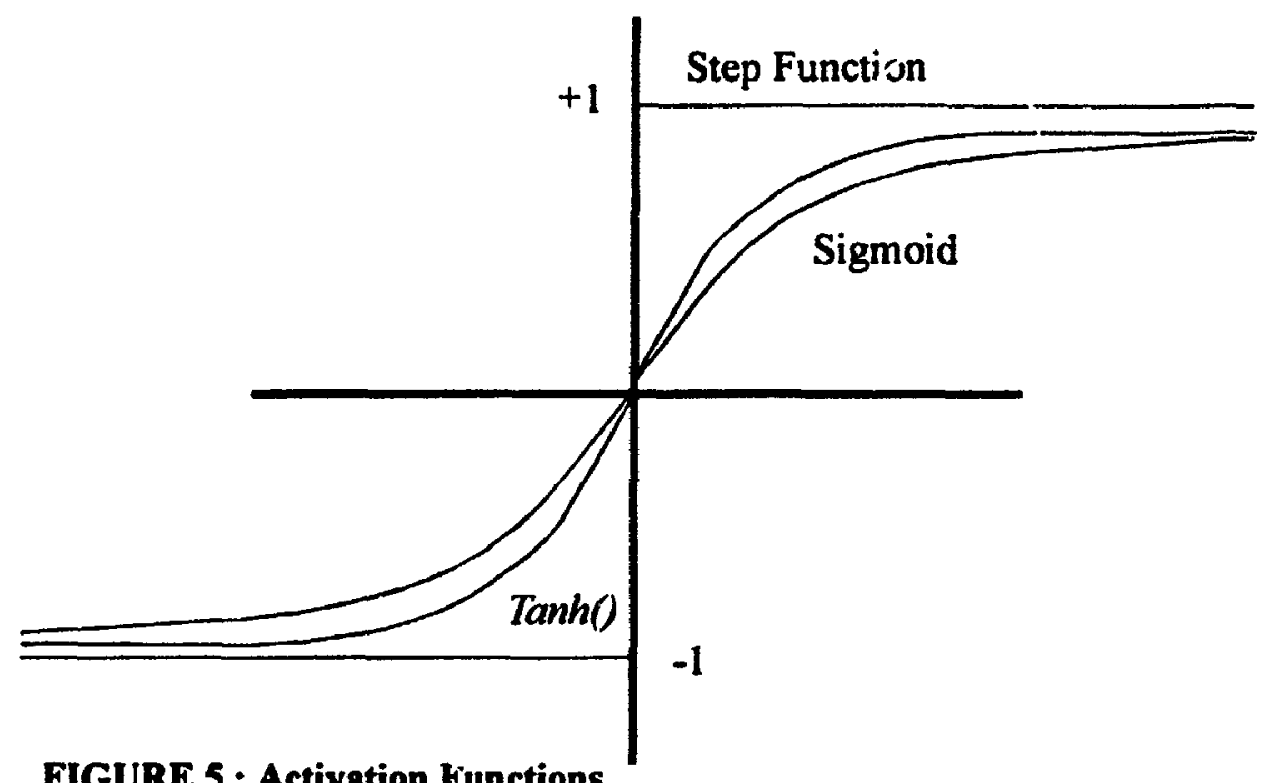

The sigmoid and the $\tanh ($ ) functions are more commonly used than the sgn() function since they are both continuously differentiable. The sigmoid is actually always greater than zero. Both a translation and linear scaling do not adversely effect learning.

both the input data and the desired output (i.e. the student and the teacher). The network learns by minimizing the error between the desired output (the teacher) and the actual output of the network (the student). In unsupervised learning, the network extracts features from the training set and the weights are self-modified to detect these features the student is self-taught. The desired output need not be explicitly known. This work focuses on networks trained using supervised learning algorithms.

\subsection{Network Topology}

So far, only layered networks with a feed-forward topology have been discussed. Modem ANNs are almost always structured in a layered format. This approach does not reflect limitations in the training algorithms or neural network theory; it merely eases design Although networks are normally designed only with connections between adjacent layers. 
short-cut connections - connections to a non-adjacent layer - can be easily handled by learning algorithms, and are used routinely by a number of different learning algorithms, including cascade-correlation which is discussed in Section 3.5.

A fully connected network contains connections between all neurons. Such a network contains lateral connections between neurons on the same layer, which allows for 'winnertake-all' decision making. The feedback connections result in a recurrent-network that allows the utilization of associative memories. The feedback allows the output of the network to be dependent on the state of a sequence of inputs, rather than a single set of inputs. Standard feed-forward networks cannot implement associative memory structures.

A general neural network architecture is shown in Figure 6.

A further variation of the layered structure is used by the cascade-correlation learning algorithm. This algorithm generates the network as part of the learning process, creating a network with complete input and output layers separated by single-neuron hidden layers (Figure 7). As previously mentioned, short-cut feed-forward connections exist between all layers. Chapter 3 examines the cascade-correlation architecture in more detail.

\subsection{Chapter Summary}

This chapter contains background information relating to neural networks, laying the foundation for the work that follows in the remaining chapters. For completeness, a brief summary of the recent history of neural network research was also presented. 


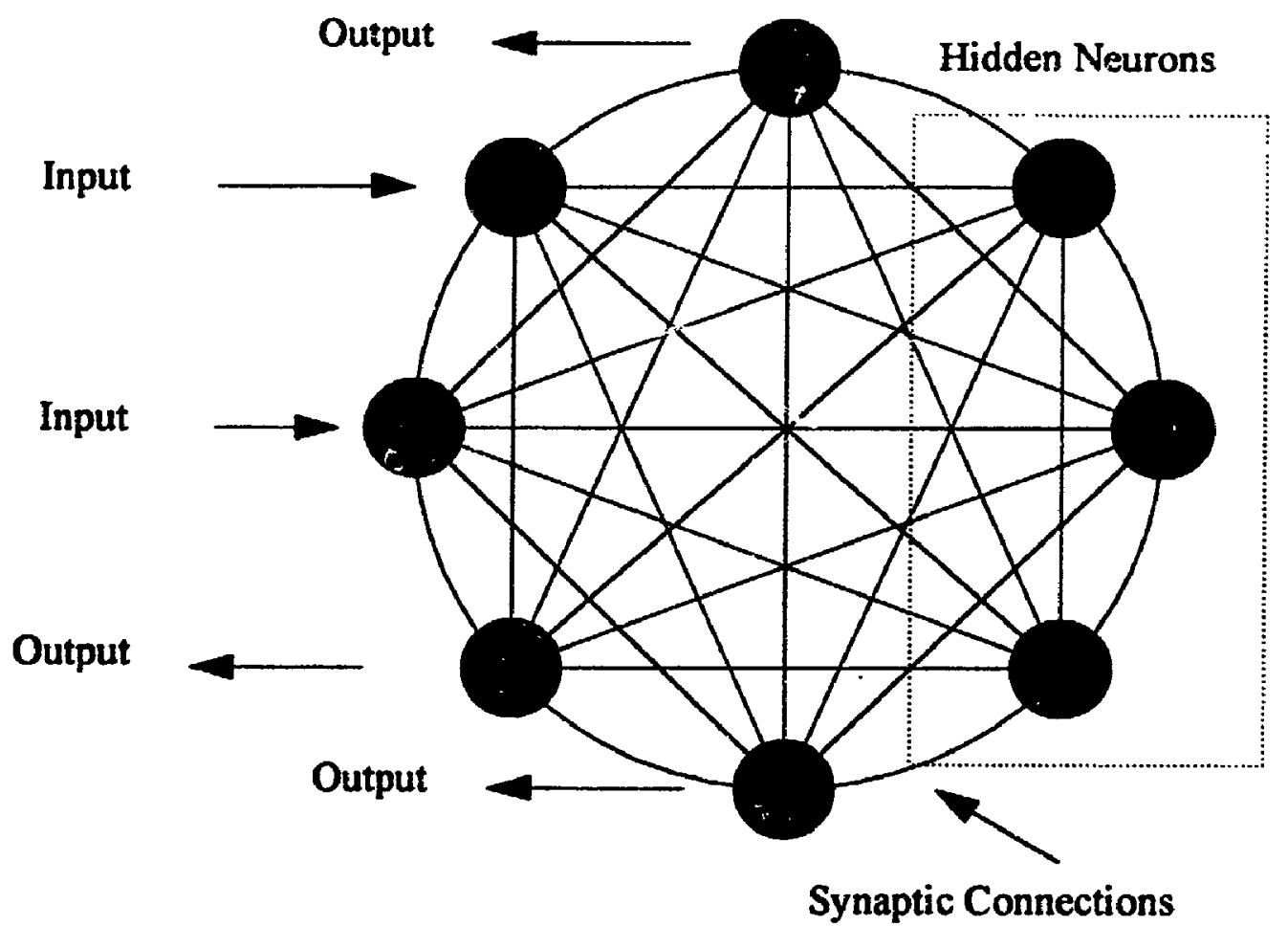

FIGURE 6 : Fully Connected Artificial Neural Network

This is the most general form of a neural network. Any type of neural network architecture may be embedded in a fully connected network, including associative memories, networks based on competitive learning, the cascade-correlation network, and the backpropagation network. 


\section{Signal Flow}

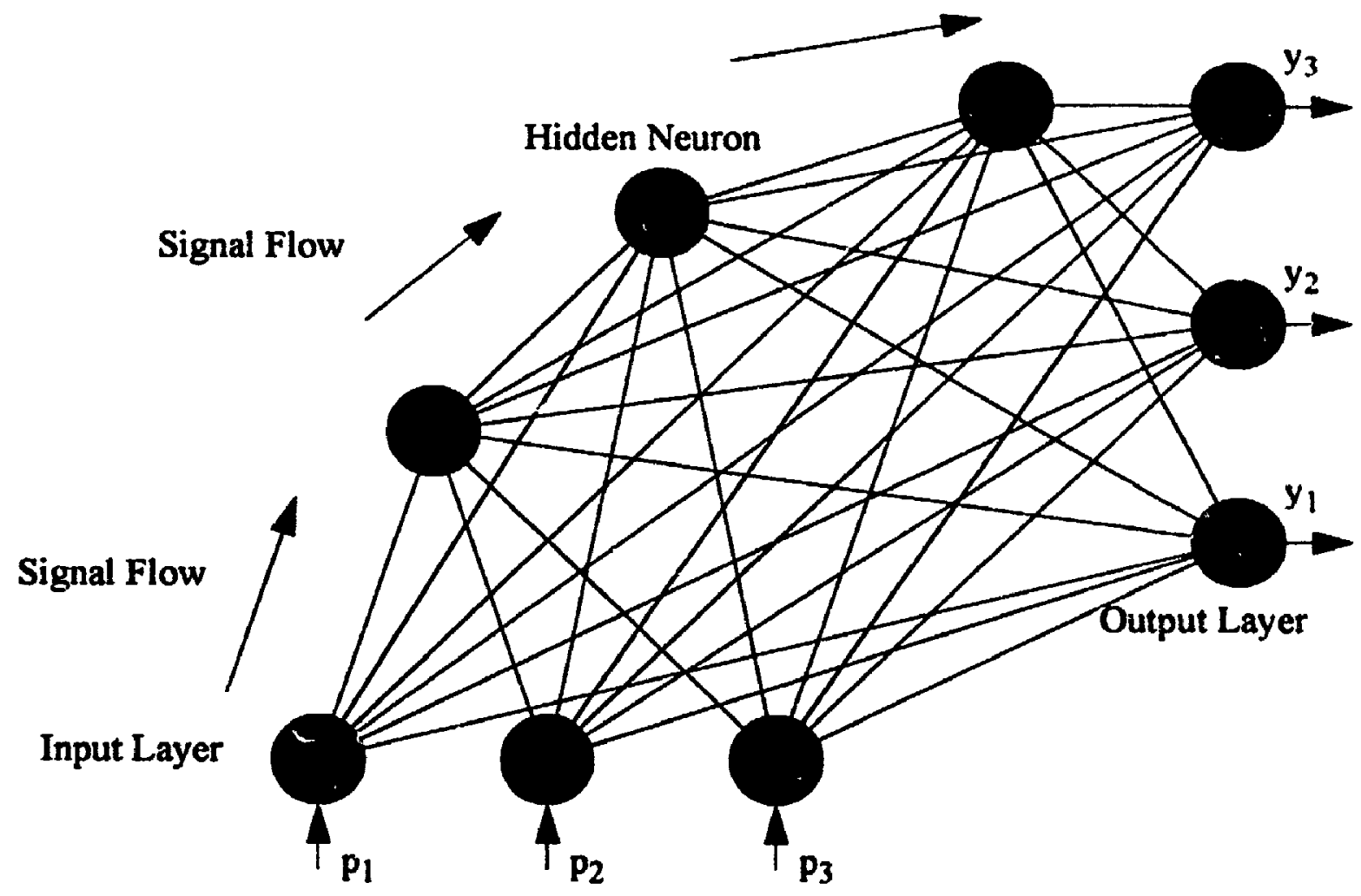

FIGURE 7 : Cascade-Cornelation Neural Network

Shown above is a three-input, three-output cascade-correlation neural network with three hidden units. Connections run from the input layer to the hidden units, as well as from the inputs directly from the output. Added hidden units receive inputs from all previous hidden units, as well as the input units and connect directly to the output layer. 


\section{Chapter 3. Neural Network Training Algorithms}

\subsection{Introduction}

This chapter examines the backpropagation and cascade-correlation neural network training algorithms. As a prelude, the delta rule, upon which both algorithms are based, is also presented. Derivations for both backpropagation ${ }^{[7]}$ and cascade-correlation ${ }^{[8]}$ are included.

The backpropagation supervised training algorithm expands on the delta rule by allowing for the creation of high-order decision surfaces. An alternate supervised training algorithm, cascade-correlation, addresses many of the deficiencies of backpropagation, also discussed in this Chapter.

Additionally, the cascade-correlation and backpropagation training algorithms are contrasted, with a focus on the suitability of VLSI technology to the implementation of these networks. The comparison shows that cascade-correlation is more suited to training neural networks implemented using VLSI technology.

\subsection{The Delta Rule-Based Training Algorithm ${ }^{[9]}$}

The delta rule is used to train single-layer networks (Figure 2) designed using neurons containing output units comprised of simple linear activation functions. The algorithm trains the network weights by minimizing, $E_{p}$, the least mean squared (LMS) error:

$$
E_{p}=\frac{1}{2}\left(d_{p}-y_{p}\right)^{2}
$$


where $d_{p}$ is the desired output for pattern $p$, and $y_{p}$ is the actual output for this pattern Although Equation 6, and the results that follow, represent the case for a single output unit, the derivation can be easily generalized for the case of multipie output units.

The weight updates are determined by setting the derivative of the LMS error proportional to the negative change in the weight update value:

$$
\Delta_{p} w_{1}=-\gamma \frac{\partial E_{p}}{\partial w_{1}}
$$

This is the delta rule. The constant of proportionality, $\gamma$, is an empirically chosen parameter called the learning rate. $w_{j}$ moderates the input from the $j^{\text {th }}$ input neuron to the output neuron. The derivative can be evaluated using the chain rule:

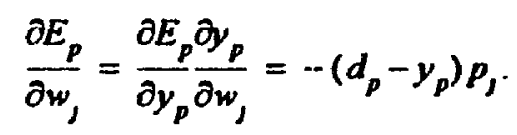

Equation 8 assumes the use of the linear activation function given by Equation 2 and the neuron transfer function of Equation 1. $p$ is the input pattern and $d_{p}$ is the target output for this pattern. Although this derivation is valid only for networks containing a single output, it clearly illustrates the processes involved with the delta rule, and can be easily extended to multi-output networks. Updates to the network weight values are calculated after each pattem has been applied to the network.

The mathematical simplicity of the delta rule makes it very attractive. Unfortunately, as discussed in Chapter 2, the delta rule can only solve classification problems that require linear separators. 


\subsection{Backpropagation Training Algorithm ${ }^{[9]}$}

The backpropagation training algorithm is by far the most widely employed and accepted supervised learning algorithm. ${ }^{[10]}$ It is used to train feed-forward networks that contain an arbitrary number of hidden units that employ non-linear activation functions. These hidden units are arranged in either an arbitrary number of layers, or in a random, ad hoc fashion.

As noted in Chapter 2, neural network research floundered for more than 15 years until backpropagation was recognized in 1986. Backpropagation was the first training algorithm that allowed the training of the incoming weights on the hidden layers when non-linear activation functions were employed. Backpropagation quickly gained popularity due to its success in a wide variety of different applications.

Backpropagation works by minimizing the error between the desired output and the actual output of the network. The error is averaged over the entire training set according to the same function as the delta rule (Equation 6) which is repeated here for the case of multiple output units:

$$
E_{p}=\frac{1}{2} \sum_{j}\left(d_{p j}-y_{p j}\right)^{2} .
$$

Similar to before, Equation 9 is the LMS error between the expected output, $d$, and the actual output, $y$, summed over each output neuron, $j$, and each test pattern, $p$, in the training set. 


\subsubsection{Learning Using the Backpropagation Training Algorithm}

Backpropagation trains a network by updating the weights to minimize the LMS error between the desired and the actial network outputs. The training occurs by presenting the entire training set to the network once, recording the LMS error associated with each input pattern, and updating the weight values based on the value of the LMS error for all input patterns. This is repeated until the LMS error is within acceptable bounds (preferably a global minimum). Each iteration is called an epoch.

The LMS error at the end of the weight update phase (epoch) can be expressed simply by:

$$
E=\sum_{p} E_{p}=\frac{1}{2} \sum_{p} \sum_{j}\left(d_{p J}-y_{p j}\right)^{2},
$$

where, as before, $d_{p j}$ is the desired output, and $y_{p j}$ is the actual output of output neuron $j$ for input pattern $p$. To minimize the total error, the weights are modified according to:

$$
\Delta w_{\imath \jmath}=-\gamma \frac{\partial E}{\partial w_{y j}} \quad, \quad \Delta_{p} w_{\imath \jmath}=-\gamma \frac{\partial E_{p}}{\partial w_{\imath \jmath}},
$$

where, as before, $\gamma$, is the learning rate. Examining Equation 11, using Equation 10, yields:

$$
\Delta w_{v j}=\sum_{p=1}^{N_{p}} \Delta_{p} w_{v j}
$$

which illustrates that the entire training pattern set is applied before weight updates occur. This ensures that the function the neural network realizes represents the entire training set - not just individual training patterns. 
Recalling the neuron transfer function:

$$
y_{p j}=f\left(s_{j}\right) \quad \text { where } \quad s_{j}=\sum_{i=0}^{N} w_{1,} y_{i p}
$$

the derivative of the LMS error with respect to the weight values can be rewritten as:

$$
\frac{\partial E_{p}}{\partial w_{\jmath}}=\frac{\partial E_{p} \partial s_{p \jmath}}{\partial s_{p j} \partial w_{\imath j}} \quad \frac{\partial E_{p} \partial y_{p j}}{\partial y_{p j} \partial s_{p j}} y_{i p},
$$

and can be further expanded by applying the neuron transfer function and Equation 10 to remove the derivatives:

$$
\frac{\partial E_{p}}{\partial w_{j}}=\left(d_{j p}-y_{j p}\right) f\left(s_{j p}\right) y_{j p} \equiv \delta_{j p} y_{j p}
$$

Equation 15 is used to minimize the LMS error (Equation 10), and thus calculate the updates for weights connected to the output layer. The updates to weight values that input to a hidden layer (a layer whose neurons connect to neither an input or an output of the network) require application of the chain rule to determine the contribution of the output LMS error to the hidden weight update. For hidden units:

$$
\frac{\partial E_{p}}{\partial y_{i p}}=\sum_{h=1}^{N} \frac{\partial E_{p} \partial s_{h p}}{\partial s_{h p} \partial y_{i p}}=\sum_{h=1}^{N} \frac{\partial E_{p}}{\partial s_{h p}} w_{i h} \sum_{h=1}^{N}\left(d_{h p}-y_{h p}\right) f\left(s_{h p}\right) w_{i h} .
$$

For a sigmoid-type activation function (Equation 5), $f^{\prime}(i)$ becomes:

$$
f\left(s_{h p}\right)=y_{h p}\left(1-p y_{h p}\right)
$$

Once the required weight updates for each synapse have been determined for all patterns 
in the training set, the weight updates are calculated using Equation 11, in conjunction with Equa.ion 15 for output weights, and Equation 1 is for input weights to the hidden units:

$$
w_{1 j} \rightarrow w_{y j}+\Delta w_{1 j}
$$

The backpropagation training procedure is outlined in Figure 8.

A wealth of information has been published about the backpropagation algorithm, for further information and examples see Müller ${ }^{[7]}$ or Kröse. ${ }^{[9]}$

1. Establish network architecture:connectivity between layers, or ad hoc; depth (\# of hidden layers); number of $\mathrm{I} / \mathrm{O}$ and number of neurons in each hidden layer.

2. Prepare training set comprised of representative input and desired output patterns.

3. Randomly initialize network weights.

4. Present training set, recording the output values for each training pattern.

5. Calculate the LMS error using Equation 10.

6. Update output weight values using Equation 11 and Equation 15 in Equation 18. Update hidden weights using Equation 11 and Equation 16 in Equation 18.

Repeat steps four to six until the network error has been reduced to acceptable levels If convergence is not achieved, adjust the learning rate and/or the number of hidden units/layers and retrain.

7. Verify learning with testing set comprised of input/output patterns not present in the training set.

FIGURE 8 : Backpropagation Training Procedure 


\subsection{Deficiencies of the Backpropagation Training Algorithm}

Although backpropagation has been successfully used to solve a wide variety of problems, it does have some serious drawbacks, as summarized in Table 1 and described below.

\begin{tabular}{|l|}
\hline Beckpropagation Learning Algorithm Limitations \\
\hline Empirically determined network size and topology \\
\hline Computationally intensive leaming phase \\
\hline Empirically chosen training parameters \\
\hline Possible for weights to enter a state where they become untrainable - 'network paralysis' \\
\hline Unjustifiably assumes all weight updates are uncorrelated - 'moving target' problem \\
\hline High numeric precision requirements \\
\hline
\end{tabular}

TABLE 1: Backpropagation Limitations

These limitations are quite general and effect implementations in both software and hardware. Critically, for fixed-hardware implementations, such as VLSI implementations, the empirically determined network size and high numeric precision requirements can have severe repercussions on the network design.

Network size is not predetermined by the backpropagation algorithm. The number of input and output units are easily chosen to accommodate the application requirements, while the number of hidden units and layers are chosen empirically, often based on the number of input units or some other 'nle of thumb'. If too many hidden units are chosen, the network will 'over-leam' the training set and will be unable to generalize when presented with input pattems not contained in the training data. Conversely, if too few hidden neurons are present, the network will be unable to learn the training set.

In a software implemented neural network, this problem can be just a nuisance. In fixed VLSI designs, however, because network size is dictated by the algorithm, this problern 
can be significant. Further design complexity must be added to the circuitry to accommodate the different network arrangements. For utmost generality, there must be a large number of neurons available for use in a first hidden layer, and different sets of neurons available for use as separate hidden layers, in addition to the neurons set aside as inputs and outputs. This can be quite wasteful. If the appropriate configuration does not have enough neurons available, some problems that could be solved using only the maximum number of neurons available on the device cannot be solved. Further, if a large number of neurons is made available, many of the neurons could remain unused. In the following section, it will be shown that the cascade-correlation algorithm does not suffer from this deficiency.

Once a network of the correct size and configuration for the application has been chosen. the network may still prove unable to adequately learn the training data. Backpropagation does not guarantee that the global minimum LMS error will be found. As $E$, the network error, diminishes during training, the network may settle in a poor local minimum. Techniques exist to rectify a poor local minimum, but these increase learning time. One technique is to increase the number of hidden units. ${ }^{[2]}$ Using this technique, it is possible to add too many hidden units, causing the network, once again, to get trapped in a poor local minimum.

Since backpropagation uses gradient descent techniques, small weight changes - actually infinitely small, are required. To increase the leaming speed, backpropagation includes an empirical parameter, the learning rate. A larger learning rate results in larger weight changes at each weight update stage. Although this can speed-up convergence at the 
beginning, if too large a learning rate is used, oscillations around the output value(s) can result, thus increasing the training time. A slower learning rate can be used to increase the probability of the networl. converging to the target solution, but the resulting learning process is extremely slow.

A further problem is so-called network paralysis. In this case, weight adjustments during training result in most of the network weights becoming large. Since the gradient is close to zero at the tails of the sigmoid (or tanh() function) the weights do not change significantly, and remain stuck. This can be solved by slowly decaying the weight values over time.

The last major deficiency of backpropagation is the moving target proble.n, which contributes to longer training times. Since all the weights are simultaneously updated at the end of each epoch, the network is drastically changed at each pass of the training data and, as a result, the training process is slowed. By altering all the weights at once, the network characteristics that the weight changes are based on are altered, causing the resultant weight updates to be non-optimal. Effectively, the algorithm assumes the individual weight updates are uncorrelated, which is an invalid assumption.

The final backpropagation deficiency is related to computability and VLSI. Training on a computer can be accomplished faster if low-precision, integer arithmetic can be tolerated. Similarly, VLSI implementations are more cost effective when low-precision weight values are used. Backpropagation cannot learn a training set unless the weight values are stored with a nituin:s'm of 13-bits precision. ${ }^{[1]}$ As a result, high-precision arithmetic must be used, which significantly slows computer simulation, and complicates VLSI 
implementation in both analog and digital designs. ${ }^{[12]}$ It will be shown in the following section that the cascade-correlation training algorithm does not suffer from this problem

\subsection{Cascade-Correlation Training Algorithm}

The cascaue-correlation algorithm is a supervised learning algorithm developed at Carnegie-Mellon University to address the deficiencies inherent to backpropagation. The backpropagation deficiencies presented in the previous section can be broadly grouped into three categories: computational complexity, high precision requirements, and empirical topology requirements. ${ }^{[8]}$

In contrast to backpropagation, cascade-correlation develops the network architecture based on the training set. The algorithm starts with a simple network containing only an input layer and an output layer. No hidden units are yet present. The network is then trained until the error reaches an asymptotic value. Further training does not result in significant reduction of the error. At this point, a candidate hidden unit is connected to the outputs of the neurons in the input layer, and to any other hidden units that have been added on previous iterations. The output of the candidate hidden unit remains decoupled from the network. The training set is once again presented to the network with the goal of maximizing the value of the correlation between the added hidden unit's output and the residual error of the network.

This is accomplished by updating the incoming weights to the candidate unit, without changing any other weight values. The output of the candidate unit is then connected to the network and the network is retrained, updating only the weights connected directly to 
the output layer until the error is once again reduced to an asymptotic value. At this point, a new hidden unit is added, using the same training procedure as before. The process of freezing weights and adding hidden units continues until network error is within acceptable bounds.

As with the delta nule and backpropagation, network error is evaluated by calculating the LMS error.

\subsubsection{A Comparison of Backpropagation and the Cascade-Correlation Training Algorithms}

Cascade-correlation reduces the computational complexity of training the network using two techniques - by removing the requirement of backpropagating errors to ti.e hidden units, and by freezing weight values once they have been trained, thereby working with a relatively small number of weights at each step.

The guesswork involved in determining network topology inherent to backpropagation is eliminated in cascade-correlation by dynamically generating the network during training. This feature alone avoids a number of the deficiencies inherent to backpropagation. Specifically, for VLSI implementations, the complexity required to implement general networks using fixed hardware is reduced. Although the network size is not known prior to the network training, the nstwork architecture is known. The network can be designed with a relatively small amount of added circuitry, enabling the implementation of either a large or a smail neural network with no changes to the actual hardware. In a VLSI implementatior, switching circuitry is necessary at the output of the neurons in the hidden layers to determine the correlation between the neuron's output value and the network 
error. By altering the addressing of these signals, neuron outputs can be routed off chip. remain disconnected from the network or be incorporated into the network.

The second major difference between cascade-correlation and backpropagation is in the way the weight values are updated. Backpropagation uses simple gradient descent while cascade-correlation uses a second-order method similar to Newton's method, ${ }^{\mid 13]}$ called quickprop (Section 3.5.2). With quickprop, fewer iterations are required to converge to a solution. Further, quickprop, unlike most second-order methods, uses an estimate of the second derivative, thereby avoiding specific calculation of the second derivative. This reduces the computational complexity of the minimization problem.

A further advantage of cascade-correlation over backpropagation relates directly to VLSI implementation - reduced numerical precision requirements. When the cascadecorrelation algorithm is used in conjunction with probabilistic weight updates |II] (described in Chapter 6), weight values represented with eight-bit precision are adequate for training and solving even those problems containing very sharp decision boundaries. such as the parity and two-spiral problems. Cascade-correlation compensates for the poor precision of each weight by adding more hidden units than would be used in a comparable network constructed using higher-precision weight values.

Also contributing to the efficiency of the cascade-correlation training procedure is the reduced $\mathrm{I} / \mathrm{O}$ requirements during training. Backpropagation updates all the weights in the network at the end of each training epoch, which can be a time-consuming task. By contrast, cascade-correlation updates only a subset of the on-chip weights - either the input weights to the candidate hidden unit, or the weights attached to the output units. 
Additionally, cascade-correlation has only one parameter, $\varepsilon$, that requires problem-specific tuning. Training the neural network thus becomes less of an art and more of a straightforward procedure, especially since the network topology (the organization of the hidden layers in a network) is not a network design parameter. This is critical for VLSI implementations, since non-idealities and component variations in the circuitry will create networks that have very different idiosyncracies when trained. The fewer parameters that require tuning, the simpler the learning procedure becomes.

The last advantage of cascade-correlation over backpropagation is the reduced numerical precision requirements of cascade-correlation. [11] Cascade-correlation can accurately perform training using less than six bits of precision for the weight values. The training procedure compensates by increasing the number of units added during training. Comparing this result to the failure of backpropagation network training with 13-bit precision weight values illustrates an additional advantage of cascade-correlation over backpropagation. The lower numerical precision requirements allow simpler circuitry, or simpler arithmetic, to be used.

Clearly, the cascade-correlation training algorithm has many merits; and addresses some of the deficiencies inherent in the backpropagation training algorithm. Especially when the problem of implementing a network using fixed VLSI hardware is considered, the cascade-correlation algorithm has definite advantages. This issue is considered in more detail in Chapter 6, where a modified cascade-correlation algorithm is presented. 


\subsubsection{Learning Using the Cascade-Correlation Training Algorithm}

The procedure of the cascade-correlation training algorithm can be separated into two phases - network error minimization, which adjusts the weights connected to the output layer; and hidden node preparation, which sets the input weight values to the hidden node by maximizing the correlation of the added hidden unit's output to the network error.

Similar to the backpropagation algorithm, coefficients at connections to the output units are trained by minimizing the LMS error (Equation 10) using Equation 15. Different from backpropagation, however, the weight updates are determined using quickprop. Quickprop approximates the error versus weighit-value curve with a parabola opening upwards. The parabola is a function of the current error slope and the $\Delta w$ values where the error slope is measured. The current weight update is made to move to the minimum point of the parabola. The $\Delta w$ definition described by Equation $1 i$ for the backpropagation algorithm is replaced by:

$$
\begin{aligned}
& \Delta w_{t}=\left\{\begin{array}{cc}
\varepsilon \frac{\partial E}{\partial w_{t}}, & \Delta w_{t-1}=0 \\
K \Delta w_{t-1}, & \Delta w_{t-1} \neq 0 \\
\mu \Delta w_{t-1}, & \text { and } \quad K<\mu
\end{array}\right. \\
& \text { where } K=\frac{\frac{\partial E}{\partial_{*}}}{\frac{\partial E}{\partial w_{t-1}} \frac{\partial E}{\partial w_{t}}} \text {, }
\end{aligned}
$$

$\varepsilon$ is a gradient-descent value, and $\mu$ is the maximum growth factor. The equation for $\frac{\partial E}{\partial w_{1}}$ is the same as the case for backpropagation (Equation 15). The subscript $w_{l}$ 's are the weight 
value at the current time step, while $w_{t-1}$ represents the weight value at the previous time step.

Weight updates for the inputs to the hidden units are calculated using quickprop as well. However, in this case, the LMS error, $E$, is replaced with $C$, the correlation of the hidden nodes output to the output error. $C$ is given by:

$$
C=\sum_{0}\left|\sum_{p}\left(y_{p}-\bar{y}\right)\left(E_{o p}-\bar{E}\right)\right|
$$

where $\bar{E}$ is the error averaged over all patterns and output units, and $E_{n p}$ is the error at each individual output unit for each pattern. The first case in Equation 19 is used to start the minimization procedure, since there is no previous value for $\Delta w_{l-1}$. The second case is used when the weight change is either too small or too large, but the slope of the error function, which is a measure of the error, is smaller than it was in the previous step. If the slope of the error function is increasing, the third case is used to ensure convergence. The parameter $\mu$ is called the maximum growth factor. If $\mu$ is too large, the weight values will fail to converge. Experiments have shown the training process to be tolerâut of a wide variety of $\mu$ vasides, however, a value of 1.75 works well for many problems. ${ }^{[13]}$

The $\varepsilon$ term is a gradient-descent value used to start the training procedure. It is the only problem-specific parameter used by cascade-correlation that requires application-specific tuning.

Although the spirit of quickprop - the minimization algorithm - is heuristic, it has proven itself with such standard benchmark problems as the two-spiral problem, the parity 
problem, and the XOR problem, to mention a few.

Using a similar derivation to that of the backpropagation, the weight update formula for the input connections to the hidden units can be shown to be:

$$
\frac{\partial C}{\partial w_{1}}=\sum_{0} \sum_{p} \operatorname{sgn}\left(E_{o p}-\vec{E}_{o p}\right) f\left(w_{1}\right) p_{i p}
$$

where $o$ and $p$ represent the sum over all output units for each input pattern, respectively. Although cascade-correlation has a more complicated training procedure than backpropagation, it is computationally less expensive. At each step in the training procedure, fewer weight updates are required. Further, none of the weight updates require the error to be backpropagated to the hidden units. This feature eases some of the computational restrictions on the other functions. In the process of training the network, with comparatively low added computational cost, the network topology is generated. In addition, using a pool ${ }^{1}$ of eight candidate units, cascade-correlation sucscisfully distinguishes two separate regions arranged as two spirals using a network containing, on average, 15 hidden tuits, requiring only 1700 epochs of training. ${ }^{[8]}$ By contrast, a backpropagation network composed of three layers, each with five neurons and shortcut connections from all the previous neurons, required 20000 epochs for the network to be trained to distinguish the two spirals. Thus, cascade-correlation is more efficient than backpropagation in terms of learning ability for complex decision surfaces. Since on-chip learning procedures are quite difficult and expensive to implement, training must be

1. A pool of candidate units can be used instead of one candidate unit. Each candidate unit is initialized with a different initial weight. The one that displays the best correlation to the LMS error is chosen for incrorporation into the network. 
1. Establish the network: Define the V/O requirements.

2. Prepare training set comprised of input patterns and desired output patterns.

3. Randomly initialize initial weights.

4. Train network (output weights) using quickprop (Equation 19) until the network error can be reduced no further. At each epoch, retain a copy of $\Delta w$ for use in the next epoch.

5. Prepare a candidate hidden unit. Randomly initialize initial weights. Train the unit by maximizing the correlation of the hidden unit output to the network error (Equation 20).

6. Add the hidden unit to the network. Freeze the input weights to the new hidden unit and connect the unit's output, through synapses, to all the output units.

Repeat steps four to six until the network error can be reduced no further. If convergence is poor, adjust $\varepsilon$, and retrain.

Once training is complete, verify the training procedure using a test set comprised of

FIGURE 9 : Cascade-Correlation Tralning Procedure

performed with the aid of a host computer. Reducing the complexity of the training algorithm allows learning to proceed more efficiently.

The cascade-correlation training procedure is outlined in Figure 9.

\subsection{Chapter Summary}

This Chapter reviewed the delta rule, the backpropagation algorithm, and the cascadecorrelation training algorithms. The limitations of backpropagation were presented and contrasted against the properties of cascade-correlation. Cascade-correlation was shcwn to be better suited to the implementation of neural networks in VLSI architectures. The most compelling advantage of the cascade-correlation algorithm is its dynamic architecture. 
which ensures that the size of a network is always sufficient for the network to be trained. The topology and size of backpropagation networks are determined by the user, and are not necessarily sufficient for the application. Further, the computing requirements required to train a backpropagation network are much higher than those for a cascade-correlation network, since backpropagation requires application of the chain-rule to propagate weight updates to the hidden layers. Other important advantages include the comparatively low numerical precision requirements of cascade-correlation over backpropagation, and cascade-correlation's faster learning characteristics. The work presented in this Chapter will be used in Chapter 6 to develop a new cascade-correlation algorithm which is even better suited to the VLSI implementation of neural networks than the standard cascadecorrelation algorithm. 


\section{Chapter 4. Neural Network Implementations}

\subsection{Introduction}

This Chapter contains a feasibility study that examines the suitability of different technology options - including both software and hardware implementations - for the ereation of large ANNs. CMOS is found to be the most favorable. Within CMOS, there are two broad design strategies - analog and digital - which are contrasted. It is shown that analog design techniques are more conducive to the design of cost-effective neural networks.

Different analog implementations of synapse, soma, and activation function circuits that have been proposed by others are examined and compared. A new, efficient architecture for the implementation of a neural network is then chosen - a floating gate differential pair for the synapse, a linear current-to-voltage converter for the soma, and a transconductance amplifier for the activation function.

\subsection{Technology}

ANNs can be implemented using many different methods (Figure 10). The most common method in use today is software simulation. Although software implementations are very flexible and programmable, their performancrs is very poor. This performance rating can be traced to the fact that a neural network gains its computational power by using a high degree of parallelism, with communication between distant units.

If an ANN is implemented on a sequential instruction execution computer, the network 


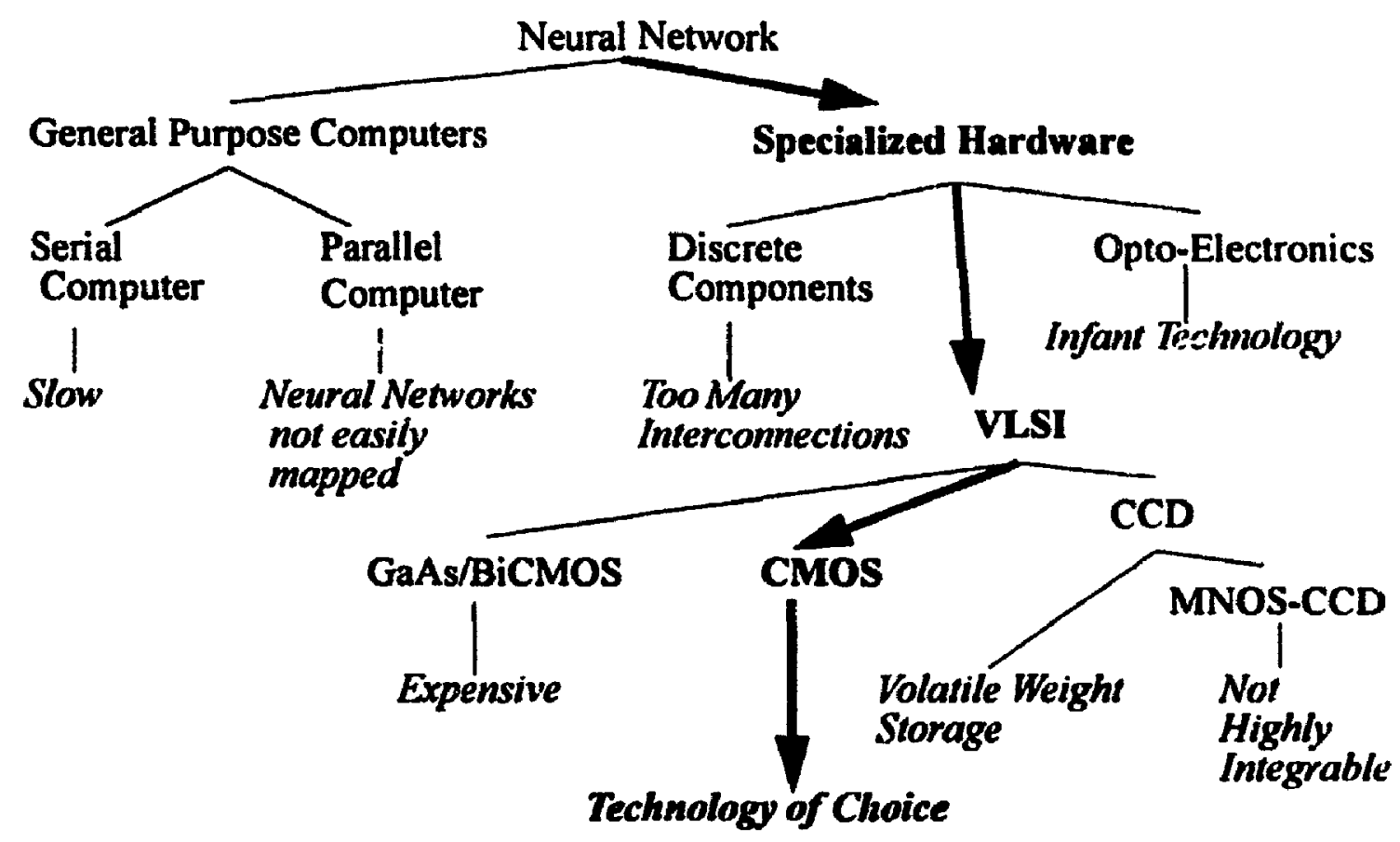

FIGURE 10 : Neural Network Implementation Technology

As indicated by the bolded arrow path. CMOS is the most cost-effective technology to use for the implementation of neural networks.

runs slowly, since each calculation must be accomplished sequentially. For example, in a modest fully trained network divided into three equal layers consisting of 16 neurons each, 512 multiplications, 456 additions, and 32 sigmoid calculations are required just to process each input pattem once. To compound the problem, the learning phase is even more computationally intensive, and often requires thousands of epochs before the network has adequately leamed the training set.

Some degree of speed advantage is gained if an ANN is implemented on a parallel computer. Due to the high level of communication between the neurons and the massive parallelism required, however, neural architectures are difficult to map onto parallel computers. If the neural network cannot be efficiently mapped onto a parallel architecture, 
little speed advantage over a single processor computer is gained.

Implementing neural networks using a fixed hardware technology leads to a number of different choices, as shown in Figure 10, compared in Table 2, and examined in more detail below.

\begin{tabular}{|c|c|c|c|c|c|c|}
\hline & $\begin{array}{c}\text { Discrete } \\
\text { Components }\end{array}$ & $\begin{array}{c}\text { Opto- } \\
\text { Electronics }\end{array}$ & GaAs & CMOS & CCD & $\begin{array}{c}\text { MNOS- } \\
\text { CCD }\end{array}$ \\
\hline Cost Enectiveness & Low & Low & Low & High & Low & Low \\
\hline Integrability & N/A & Low & Low & High & High & Medium \\
\hline Flexibility & High & Low & Low & Low & Low & Low \\
\hline
\end{tabular}

TABLE 2: Comparison of Flred Hardware Technologles

The limitations of software-based neural networks implemented on general purpose computer hardware can be overcome by using specialized hardware. Due to the large number of both interconnections and simple components in a neural network, specialized VLSI hardware is preferable to a discrete design approach.

Using a discrete design approach, subcircuits can be implemented on separate integrated circuits. Although this makes the design of each IC quite simple, it leads to complication $s$ at the board level design. Similar to parallel computer architectures, a large number of connections are required between components. Additionally, for a large network, a large number of ICs is required to implement the network, increasing cost, and reducing reliability. A compromise can be reached by implementing large blocks of circuitry on each device - such as a synapse chip, and a neuron chip, thus reducing the amount of interconnections required between devices, and the total number of devices required to implement the network. This technique has been used by a number of researchers, 
including Lansner et.al. ${ }^{[14]}$ The following discussion applies to situations where the entire network is implemented on one IC, or alternatively, distributed over two or more ICs.

Optoelectronics is still in its infancy. It does not allow the development of large, highdensity circuits, and is therefore not considered as a viable technology option. Further. because the technology is so new, the base of prior knowledge upon which to build upon is quite small.

Integrated VLSI designs can be accomplished using any existing process technology. however some are more suitable than others. For example, GaAs (Gallium Arsenide) processes allow the design of very high speed neurons and synapses. GaAs technology. however, is relatively immature and very expensive, making it a poor choice for the lowcost production of large networks.

Much of the discussion pertinent to GaAs implementations also apply to BiCMOS (Bipolar Complementary Metal Oxide Semiconductor) technology, another potential highspeed technology, which is a hybrid of CMOS and bipolar. BiCMOS offers high-density. high-speed designs, but at the cost of added processing complexity. Since subcircuit speed is not a critical design concern, it is believed that the added circuit and processing complexity cannot be justified at the present time.

CMOS is an adequate technology since it allows for complex digital circuitry and sufficient analog circuitry. ${ }^{[15]}$ Further, in a double-polysilicon CMOS process, floatinggate technology can be implemented, allowing for non-volatile programmable storage of on-chip weights. ${ }^{[16],[17]}$ 
CCDs (Charge-Coupled Devices) are another technology that is being explored for VLSI implementation of ANNs. CCD technology allows simple storage and manipulation of volatile synapse weight values. Although non-volatile weights have been developed using MNOS-CCD (Metal Nitride Oxide Semiconductor-CCD) technology, ${ }^{[18]}$ CMOS is the technology of choice, since it allows for higher levels of integration.

\subsection{CMOS Design Criteria}

The choice of CMOS implementation technology allows the design to be based on either digital or analog circuitry. Table 3 compares digital and analog implementation strategies for the subcircuits necessary to implement a neural network - inner product circuits (multiply and addition), synaptic weight storage circuits, and activation function circuits.

\begin{tabular}{|c|c|c|c|c|c|c|c|c|}
\hline & \multicolumn{4}{|c|}{ Digital } & \multicolumn{4}{|c|}{ Analog } \\
\hline & \multicolumn{2}{|c|}{ Inner Produet } & \multirow{2}{*}{$\begin{array}{l}\text { Difital } \\
\text { Welght } \\
\text { Storage }\end{array}$} & \multirow{2}{*}{$\begin{array}{l}\text { Activation } \\
\text { Function }\end{array}$} & \multicolumn{2}{|c|}{ Inner Product } & \multirow{2}{*}{$\begin{array}{l}\text { Andog } \\
\text { Weight } \\
\text { Storage }\end{array}$} & \multirow[b]{2}{*}{ Act fo. } \\
\hline & $x$ & + & & & $x$ & + & & \\
\hline $\begin{array}{l}\text { Cost } \\
\text { Enteetiveness }\end{array}$ & poor & poor & poor & poor & high & high & medium & high \\
\hline Flealbility & poor & poor & medium & high & medium & high & medium & high \\
\hline Integrablllty & high & high & high & high & medium & medium & medium & medium \\
\hline Prociblon & high & high & high & high & medium & medium & mediu'n & poor \\
\hline Pertordenes & poor & high & medium & poor & high & high & medium & high \\
\hline
\end{tabular}

TADLE 3: Digital ve. Andlog Implementation Strategles

Digital circuitry is not cost effective for implementing any of the four functions required in a neural network. Multiplying and adding high-precision numbers requires a large amount of circuitry and routing, thus resulting in a large die area. Although digital circuitry can achieve arbitrary numerical precision, this too is accomplished at the expense 
of area. For maximum efficiency, these circuits must be replicated at each synapse, and thus are not feasible for large designs.

The main advantage of digital circuitry over analog circuitry is that it is highly integrable, since it is relatively insensitive to device mismatch. Analog circuitry, on the other hand, allows the simple implementation of arithmetic functions at a cost of reduced precision, partially due to device mismatch.

However, analog circuitry can accomplish the inner product calculations quite efficiently. since multiplication can be achieved using the inherent properties of the MOS transistor. Further, the additions can be quickly accomplished by summing currents at a node. Using digital techniques, multiplication requires multiple clock cycles, and the multiple additions required to perform the irner product must be done sequentially.

Digital weight storage techniques can be accomplished quite simply. As in the previous cases, however, they are area intensive. Storing an eight-bit number requires eight memory locations and eight data lines. Storing the weight as an analog value requires only one memory lxcation and one analog data line. The disadvantage of using analog memory techniques is that arbitrary precision is not possible. Current CMOS technology can accomplish only about 10-12 bits of precision whun storing information on a capacitor for a short time, which is sufficient for only a few learning algorithms. Lnnt et. al ${ }^{[19]}$ report measured capacitor discharge rates of $2 \mathrm{mV} / \mathrm{ms}$ for $320 \mathrm{fF}$ capacitors in the $3 \mu \mathrm{m}$ SACMOS process, with a single discharge path through a minimum-sized transistor. Further discussion of storage techniques follows in Section 4.5. 
The activation function is most simply implemented using analog circuitry. The sigmoid most commonly used by backpropagation can be replaced with the hyperbolic tangent function, which has similar properties and can be approximated using the differential pair described in Section 4.9. Using a digital circuit to implement the sigmoid function requires severe approximations if it is to be implemented using a minimal area. A digital high-precision sigmoid can only be implemented using complicated circuitry or by storing discrete values in a look up table.

Although a digital-type implementation allows high-precision, parameter-invariant computations, analog implementations are the preferred implementation, as shown in this section. Analog implementations allow only low- to medium-precision operations, but provide them in a very cost-effective manner.

\subsection{CMOS Implementation Strategy}

Choosing a design strategy is critical to the realization of an economical ANN. The most important analog VLSI implementation issues are presented in Table $4^{[20]}$ are discussed in detail beilow.

\begin{tabular}{|l|l|}
\hline Technolosy Constraint & Resulting Problem \\
\hline Chip Area & Reduced yield \\
\hline Parameter Variation & Poor performance, reduced yield \\
\hline Leakage Current & Increased noise, reduced yizld \\
\hline Power Dissipation & Low reliability, reduced circuit performance \\
\hline Reduced Supply Voltege & Scalability to deep sub-micron feature sizes \\
\hline Interconnect and Fan-out Delay & Slow circuit operation \\
\hline I/O Pad & Communication to outside world limited \\
\hline
\end{tabular}

TABLE 4: CMOS Technology Constraints 
Silicon chip area is an important factor in any IC fabrication technology. As chip area increase, the probability of a defect occurring on the die increases exponentially. Although neural networks are fault tolerant due to their distributed processing architecture, the presence of fauits can render a die inoperative or increase learning time. A defect which, for example, generates a stuck fault at a neuron output, leaves all the synaptic inputs to that neuron untrainable. Although during training those weights will be updated, they never result in a change to the output, thus increasing training time, since time is spent training useless synapses. If a significant number of neurons are disabled in such a manner, then the network becomes untrainable. It is therefore vital to design the network neurons and synapses so that each individual sub-circuit utilizes a minimum area.

Another factor contributing to yield reduction (the ratio of good devices on a wafer to all the devices on the wafer) is parameter variation. Most device parameters, including transistor gain and $V_{T}$, vary in value for transistors across both individual die and across wafer batches. To function as expected, the design must be tolerant of these variations. Whenever possible, critical functions should be implemented as a ratio of two devices, rather than as an absolute value.

Transistor leakage currents result in excess noise, reducing the dynamic range of highperformence circuits on the chip To ensure yield and performance remain uncompromised, transistor leakage current should be taken into account during detailed system design. Additionally, power consumption must be minimized if large designs are to be implemented. With high power consumption, the local temperature on the die is increased, resulting in both reduced reliability and performance. 
For compatibility with sub-micron technologies, a design that can operate with a 3.3 Volt supply should be used. At sub-micron dimensions, the electric field present when $V_{D S}=5$ Volts on a minimum-geometry NMOS (N-channel MOSFET) transistor generates hot electrons, which embed themselves in the oxide, resulting in permanent $V_{T}$ shifts, and eventual device failure. Even when designing circuits in a 5 Volt process, configurations which are compatible with 3.3 Volt supplies should be used to ease design scaling to reduced feature size technologies.

Interconnect delay plays a role in any large design. As device sizes shrink and die sizes remain the same size or grow, the relative length of the interconnections increase. Further, interconnect dimensions have not scaled as fast as transistor dimensions, resulting in interconnect capacative and resistive delays becoming a greater design challenge. Line lengths should be minimized, or if this is not possible, timing should be relaxed to allow the line time to sufficiently charge before sampling. A more efficient method to overcome the interconnect problem is to use current mode signals on long lines. With a current mode signal, the voltage on the line varies only slightly, thereby allowing signal transmission to take place more efficiently, since the delays caused by charging the interconnect capacitances are reduced. Another alternative is to use BiCMOS. However, BiCMOS significantly increases the complexity involved to scale the design to sub-micron feature sizes, as well as increases manufacturing cost and complexity.

In addition to interconnect delays, the high fan-out inherent in neural architectures places further constraints on the design. The neuron output and, in the case of an analog design. the summation node, have a large number of transistors attached. In analog designs the 
summation node is typically a current mode signal. ${ }^{[21]}$ The neuron output could be current mode as well. Another alternative is to lay the circuit out such that all the synapses connected to the neuron output are physically located close to the neuron, as in Figure 11 , thus minimizing the effect of interconnect delays if the synapse output is a current-mode signal. The aggregate load of the synapse input transistors may still be large, thus requiring the neuron output to have a fairly high drive capability. Since the number of synapses is typically an order of magnitude (or more) greater than the number of neurons. the silicon area used to implement the neuron output function is not a critical design parameter.

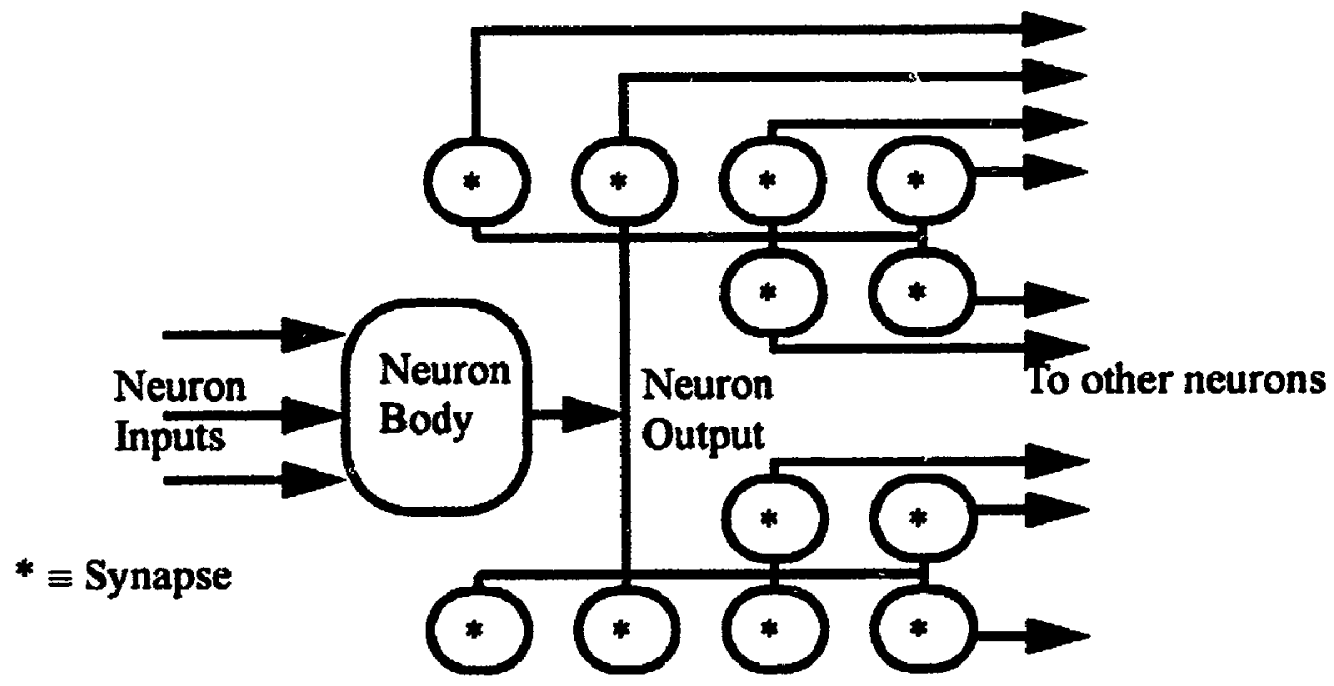

FIGURE 11 : Local Layout Style for Large Neural Netwosk

If the neuron outputs a voltage mode signal, and the synapses output a current mode signal, interconnect delays can be minimized by placing the synapses connected to the output of a neuron near that neuron.

The final design criterion deals with interfacing to the outside world. Neural networks can require a large number of inputs and outputs. Although in recent years, pin grid array (PGA) packaging technology has allowed witips to be developed with hundreds of $1 / O$ 
pins, ${ }^{|22|}$ the cost of these packages can be quite high. In a digital network, the interface to each input and output neuron requires one signal for each bit of precision. A number of options exist. First, a databus architecture can be implemented where the inputs and the outputs share a common bus, and the inputs and the outputs neurons are addressed when valid data is on the line. A better option is to use analog data lines, where only one signal per input/output is required. This reduces the complexity of the timing.

\subsection{Weight Storage Considerations}

The optimal weight storage method chosen for a given implenentation is dependent on both the learning algorithm and the anticipated applications. Numerical precision concerns arise from the limitations of the learning algorithms. Since, in general, numerical precision in both digital and analog designs can be increased only at the expense of silicon area, such precision cannot be made arbitrarily accurate, since large networks will become impractical.

The other two significant design issues for synaptic weight storage are ease of programmability, and whether volatile or non-volatile storage should be used. These issues are partially dependent on the anticipated application. For greatest generality, the storage elements should be non-volatile and easily programmable.

\subsection{Area Requirements}

The area required for storage of each synaptic weight elem ?nt ultimately limits the size of the neural network that can be practically implemented. Since each neuron is interconnected with many other neurons through individual synapses, there will be many 
more synaptic connections than neurons. The human brain is believed to have in the order of $10^{9}$ neurons, each interconnected to between $10^{3}$ and $10^{6}$ other neurons. ${ }^{[23]}$ Modern ANNs cannot yet approach this high level of integration. For a layered ANN segmented into $N$ layers of $M$ neurons a total of $(N-1) M^{2}$ synapses are required to fully connect each layer. To generate a fully connected network a total of $[(M-1)+M(N-1)] M N$ synapses are required. Due to the high cost function in implementing fully connected networks, little effort is being made in their implementation. Although the cost funciion for a layered neural network is lower, the number of synapses still increases as the squaie of the number of neurons making synaptic area the critical parameter.

\subsubsection{Synaptic Storage Elements and Numerical Precision}

Many learning algorithms require the weight value to have very high precision in both the training and recall phases. Backpropagation, for instance, requires the weights io have a minimum of 12-bits accuracy for the training phase although, once trained, the network can typically operate correctly if the weight precision is dropped below eight bits. ${ }^{[I I}$ The minimum equivalent numerical precision (dynamic range) required by an integrated analog implementation will be larger due to non-idealities introduced by the physical implementation, since the actual storage element is not the only source of precision degradation.

Although an analog design strategy has been determined to be better suited than a digital design strategy for integrated neural network design, some digital storage techniques are considered below since some researchers, including Mueller et. al., ${ }^{|24|}$ use analog design techniques with digital-iype storage. 
Using digital techniques, SRAM- (Static Random Access Memory) or DI AM-type (Dynamic Random Access Memory) cells could be used for volatile storage, with one memory location for each bit of required precision. The DRAM cell can be implemented using one transistor, while each SRAM cell typically requires six transistors. The size advantage of using DRAM type storage comes at the cost of added design complexity. The information stored on a DRAM requires periodic refreshment, since the charge stored gradually leaks away. In a small network, the area required by the refresh circuitry, as well as the added design complexity, may offset the area savings gained by the use of a more compact storage element. In a large ANN implementation, however, the use of SRAM storage cells can become impractical. For a modest network containing 1024, 16-bit synapses, $16 \mathrm{~K}$ of storage space is required.

The digital-type storage approach requires digital-to-analog converters or significant busing to interface with the (analog) neural network. Volatile analog storage is not a viable option, since the stored charge will leak away with time. The refresh rate must be at least

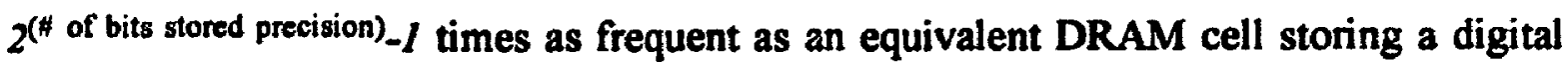
value. Unlike conventional digital DRAM technology, a simple comparator cannot be used to refresh the synapse, since the capacitor stores an analog value. The complexity of the refresh circuitry clearly eliminates the advantages gained through the use of a smaller storage element.

Additionally, if a charge redistribution scheme is used, the architecture must allow the weight storage to be dependent on capacitor ratios to ensure maximum precision, since the ratio of capacitors can be controlled with high precision (up iv $0.1 \%$ deviation), whereas 
large deviations can be present in absolute capacitances.

A further reduction in design complexity can be achieved by using non-volatile storage elements. Recently, there has been a significant research effort into the use of EEPROM (Electrically Erasable Programmable Read Only Memory) technology to store analog values. Using an EEPROM-type structure to store the weights allows the weight storage to occupy a small area without the added design complexity of having to periodically refresh the weight value. A second very impor"ant advantage is that once the weight values are programmed into a network with EEPROM type storage, they remain even when the device is powered down. An ANN whose weights are stored using volatile storage techniques requires the weights to be re-loaded every time the device powers on. Nonvolatile storage is clearly preferable.

\subsubsection{Synaptic Restrictions Imposed by Application Requirements}

If the operating environment for the neural network is known at design time, the synaptic weight storage scheme can be chosen appropriately. If the network is to operate in an environment such that the synaptic weights will be programmed once and remain fixed, a non-volatile storage scheme would be the most efficient design style. If the network is to be reprogrammed frequently, then a volatile storage scheme might prove adeq:tate. For example, if the network is to operate as asi accelerator board in a computer, the weights can be efficiently downloaded when required, thus reducing the fabrication complexity of the device.

Further, if no programmability is required for the network, it may prove sufficient to hard- 
wire the synaptic weights into the device as ratioed resistors, or in a ROM if a digital storage scheme is to be used. However, this type of implementation is susceptible to parameter variations, since training cannot be performed using the physical neural network.

The most stringent requirements on the type of storage scheme used in the network are imposed by the learning architecture. As noted previously, backpropagation learning requires a minimum of 12-bit precision. This cannot be accomplished using a simple capacitor to store the analog weights. If an alternate algorithm was found that did not have such stringent precision requirements, such as the cascade-correlation algorithm, ${ }^{[11]}$ capacitively stored weights could be adequate.

Since the size of the synapse ultimately limits the size of the network, the smallest possible synapse should be used. This excludes the use of digital storage techniques.

Each neuron circuit is composed of three main components: the synapse (multiplication), the soma (addition), and the activation function (sigmoid or $\tanh ($ )). In Sections 4.7 to 4.9, each component is considered separately.

\subsection{Synapse Circuits}

Using analog circuitry there are many methods of implementing inner-product circuits, most of which operate on the same principle, performing the multiplication and then summing on a single node. Conceptually, the simplest method of implementing such a circuit is to use an operational amplifier-based summation circuit, as shown in Figure 12. 


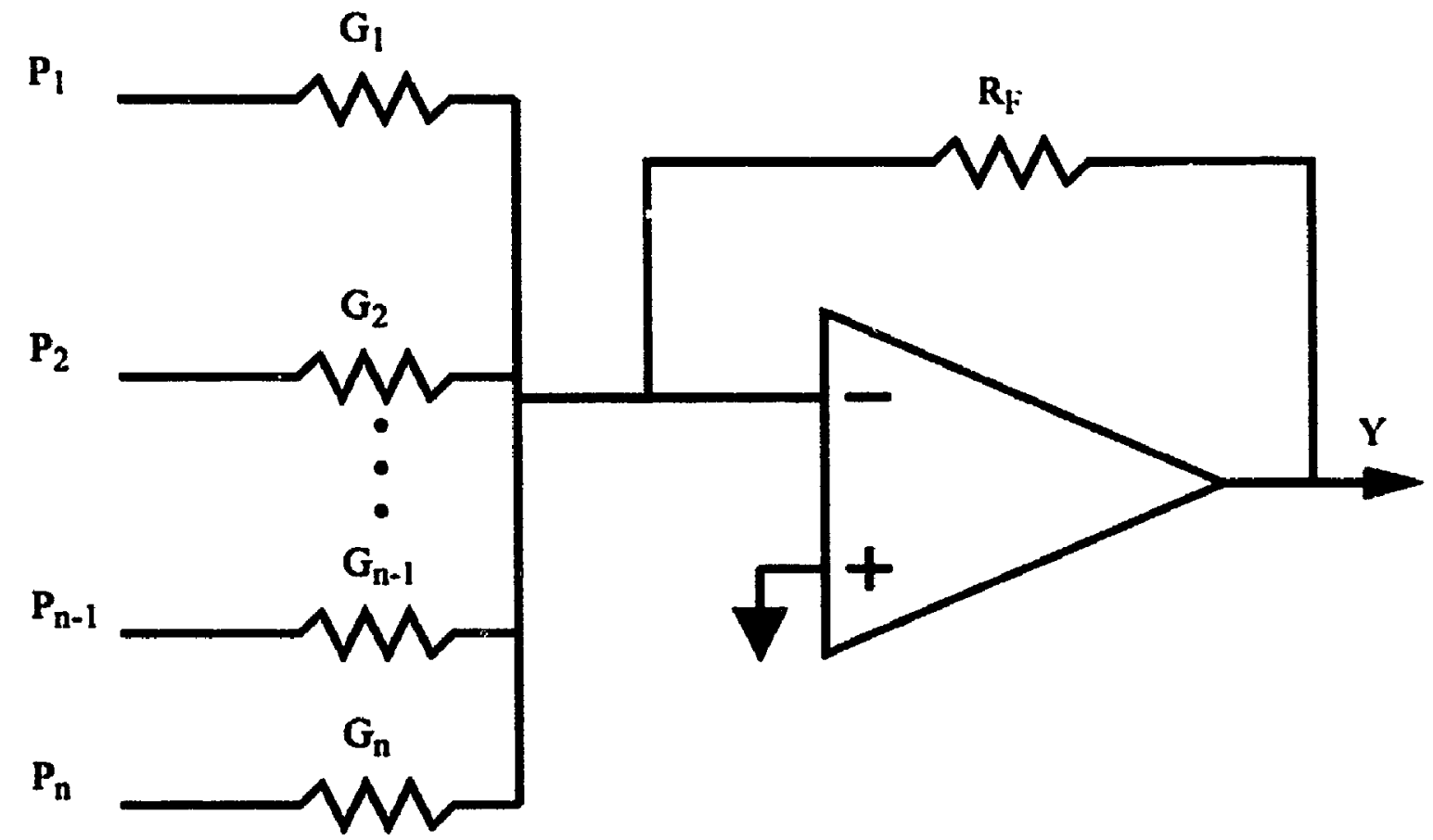

FIGURE 12 : Op-Amp-Based Inner Product Cincuit

This circuit multiplies the input pattern with the weights (stored on the resistors) and performs a weighted sum at the input to the operational amplifier.

The transfer function of this circuit is:

$$
y=\sum_{j=1}^{n} p_{j}\left(-R_{F} G_{j}\right)=\sum_{j=1}^{n} p_{j}\left(-\frac{R_{F}}{R_{j}}\right) .
$$

The weights are stored in the input resistors as the admittance, $G$, of the resistances.

This implementation strategy is useful for small, fixed networks. From Equation 23, it is evident that the absolute value of the weights is not as important as the ratio of the weights, which can be controlled more precisely. Although the weight values can be defined accurately (within 1 percent), this approach does not allow for easy programmability. Resistors, once implemented, are fixed. Theoretically, if the resistors are designed with taps, they can be programmable. However, this adds significantly to the 
routing complexity of the circuit. If higher precision is required, a switched-capacitor resistor replacement can be used, as illustrated in Figure 13.

Although switched-capacitor neural network impismentations have been reported, ${ }^{[25]}$ programmability is limited. Since the effective resistance of the switched-capacitor is $R=1 / f C$, the resistance can be programmed by changing the clock frequency or by changing the capacitance. On a neural network chip, changing the clock frequency is not feasible, since separate clocking would be required for each synapse $\left(z^{\#}\right.$ of bits fi precision +1 different clocks). The capacitance can be altered using a technique similar to that shown in Figu:e 14. However, the advantages of analog type storage are iost, since the resistance is programmed using digital-type addresses.

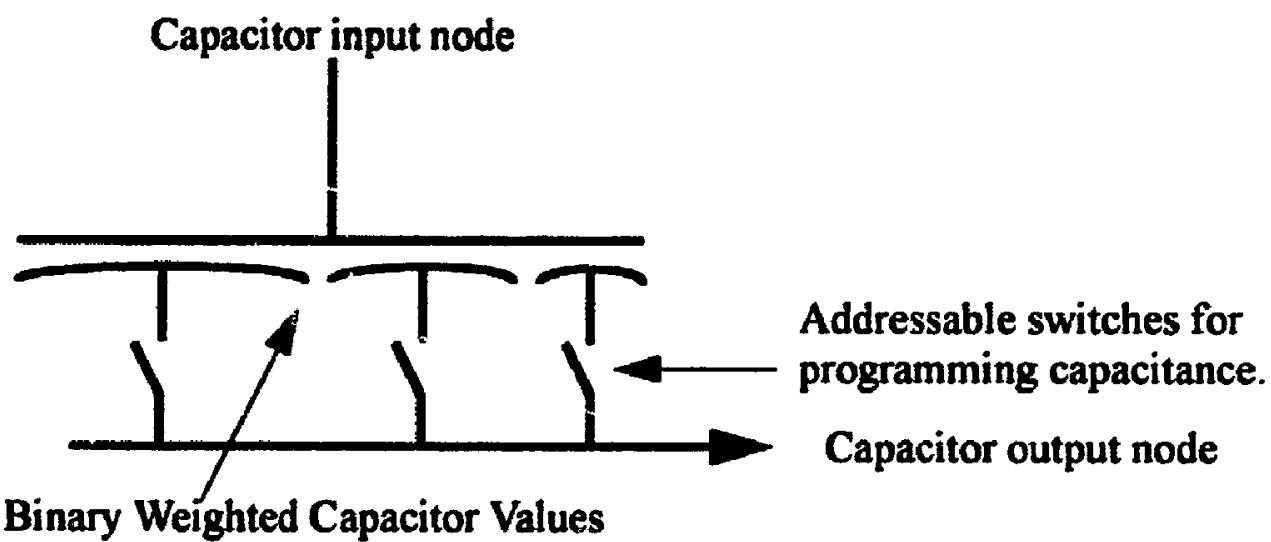

\section{FIGURE 14 : Programmable Capacitor}

For $2^{n}$ programming levels, $n$ switches and control signals are required in addition to the four switches and clocks required for each switched-capacitor resistor. Since the capacitance for each capacitor is based on the area, the capacitors can be size ${ }^{A}$ to yield binary values.

The problem with both the switched-capacitor and resistor implementations is the difficulty in programming, and in the large amount of area required by each synapse. The fixed-resistor implementation can be modified as in Figure 15 to use MOS devices biased 
a)

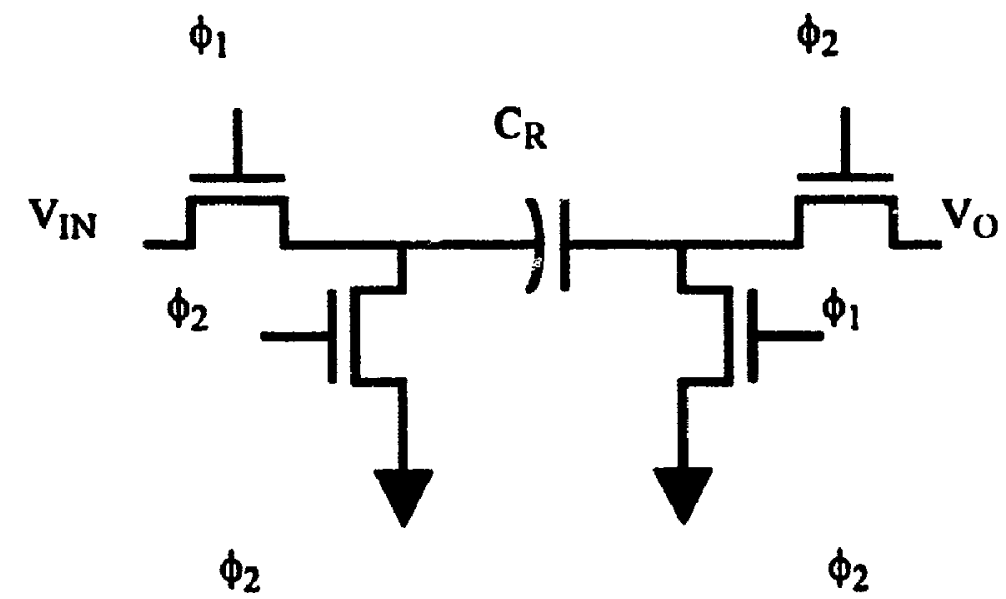

(b)

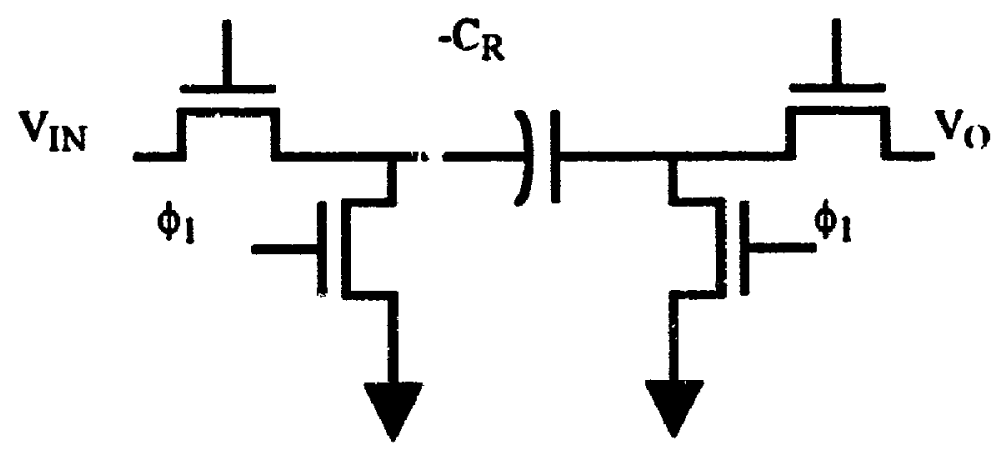

(c)

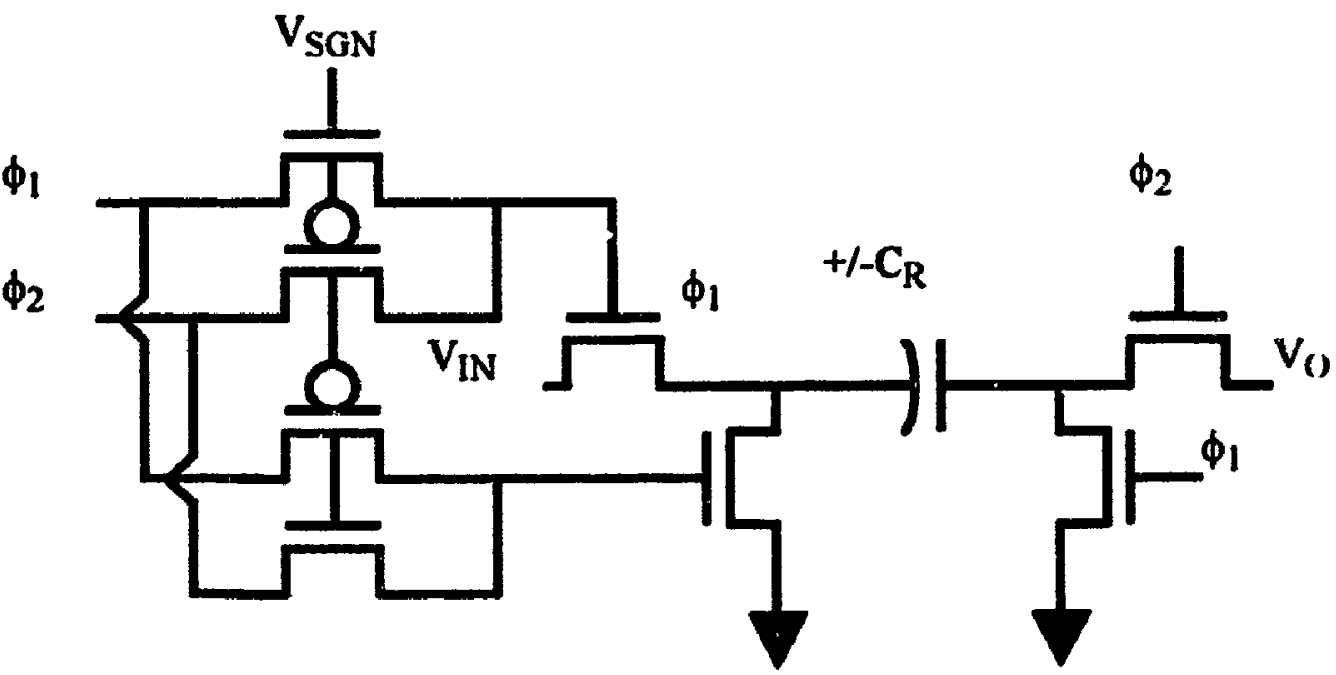

FIGURE 13 : Switched-Capacitor Resistors ${ }^{[26]}$

(a) A positive resistance; (b) negative resistance; (c) programmable positive or negative resistance $\phi_{1}$ and $\phi_{2}$ are non overlapping clocks - only one is active at a time. The sign bit, $V_{S G N}$ is a static signal The absolute value of the effective resistance of the switched-capacitors can be modified by varying either $C_{R}$ or the clock frequencies. 
in the linear region. Using the Shichman and Hodges model ${ }^{[27]}$ for a CMOS transistor biased in the linear region;

$$
I_{D S}=\beta\left(V_{G S}-V_{T}\right) V_{D S}-\frac{V_{D S}^{2}}{2} \quad, \quad V_{G S}<V_{D S}-V_{T},
$$

where $\beta$ is the transconductance parameter, the effective resistance of the transistor can easily be shown to be:

$$
R_{D S}=\frac{1}{\beta\left(V_{G S}-V_{T}\right)}
$$

Although this allows programmability by altering the control voltage, $V_{G S}$, this results in low dynamic range due to the small linear region inherent to MOSFETs (Metal Oxide Semiconductor Field Effect Transistor).

A different technique employs transconductance amplifiers to implement the synapses. Although this technique also requires a relatively large silicon area, higher precision can be achieved. Lee and Sheu ${ }^{[28]}$ describe an architecture where the weights are stored on capacitors, biasing one of the inputs to a differential transconductance amplifier as shown in Figure 16.

The multiplication is performed between the bias voltage on the current source - which is connected to a neuron output - and the weight voltage. It is straightforward to show for Figure !6:

$$
I_{1}-I_{2}=\beta_{2}\left(\frac{4 I_{M 1}}{\beta_{2}}-w_{1 j}^{2}\right)
$$




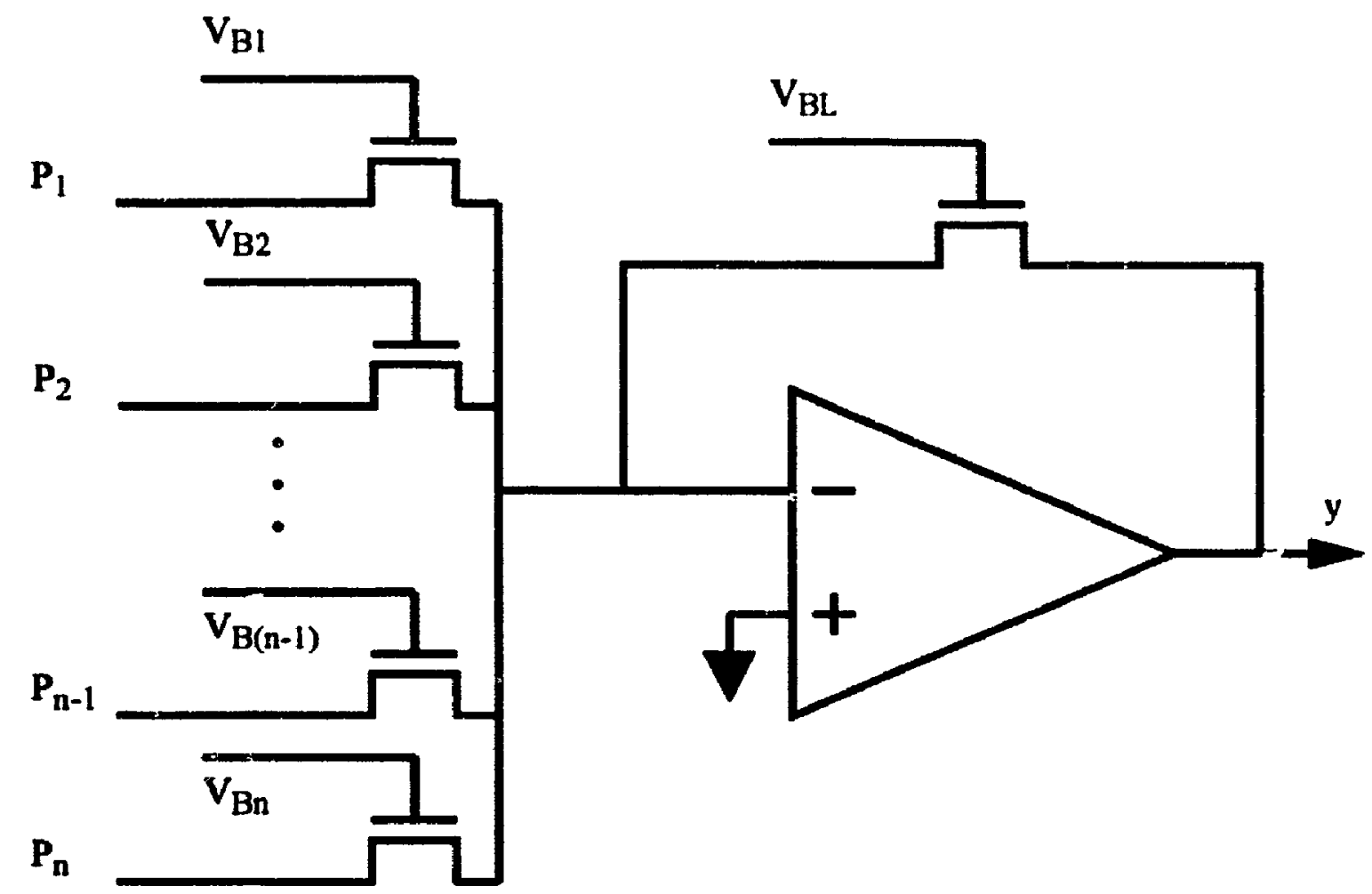

FIGURE 15 : MOSFET Resistor-Based Inner Product Circuit

This sircuit is identical to the resistor implementation except, that it allows programmability through the independent gate connections.

where $\beta_{2}$ is the transconductance factor of transistor $M_{2}, I_{M l}$ is the current through transistor $\mathrm{M} 1$ and it is assumed the $\mathrm{M} 2$ and $\mathrm{M} 3$ are matched. A reasonable approximation of a true multiply exists if the circuit is designed such that:

$$
w_{1,}^{2} " \frac{4 I_{M 1}}{\beta_{2}}
$$

The outputs of the synapses are connected to the neuron body and the other synapses. As with the resistor and MOSFET implementations, this common node is a current-mode signal and can be treated as a virtual ground. 


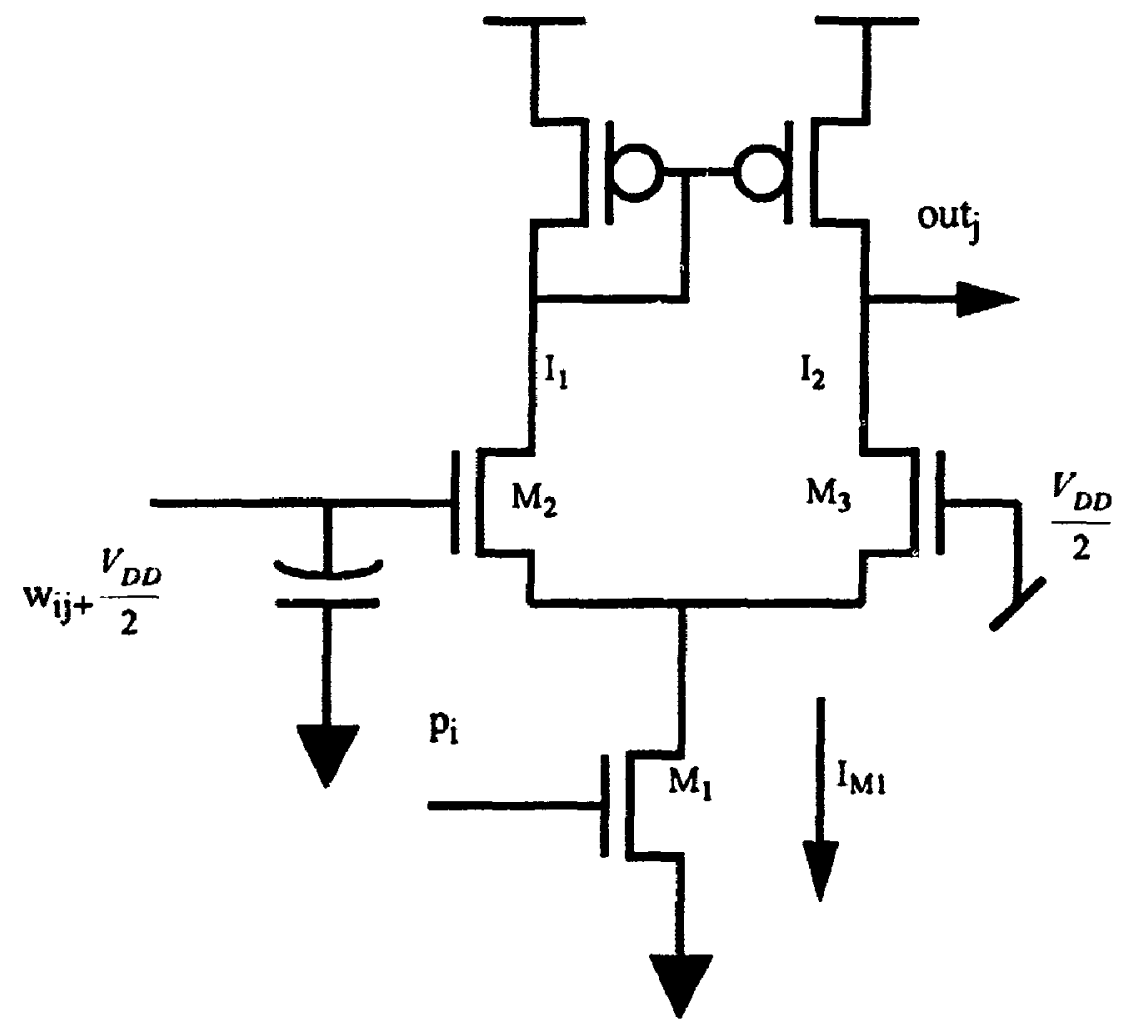

FIGURE 16 : Transconductance Amplifier-Based Inner Product Circuit

The input signal, $p_{l}$, is multiplied by the interconnection weight, $w_{l j}$. This multiplication is an approximation of a true multiply.

A variation on the transcorductance amplifier-based synapse implementation reduces the number of iransistors required to implement the synapse to only three per synapse, with the load devices shared between all the synapses input to a neuron. The transconductance amplifier of Figure 16 is replaced with just the input differential pair and the current source. The weight storage can be implemented with a capacitor as above, or as by Bibyk et.al..$^{[21]}$ and Thomsen et. al. ${ }^{[17]}$ by using a floating gate tranisistor as one or both of the transistors in the input pair (Figure 17).

The floating gate-based differential input pair removes the requirement for a separate storage mechanism. A single-bias voltage can be used for all the input pairs in the network 


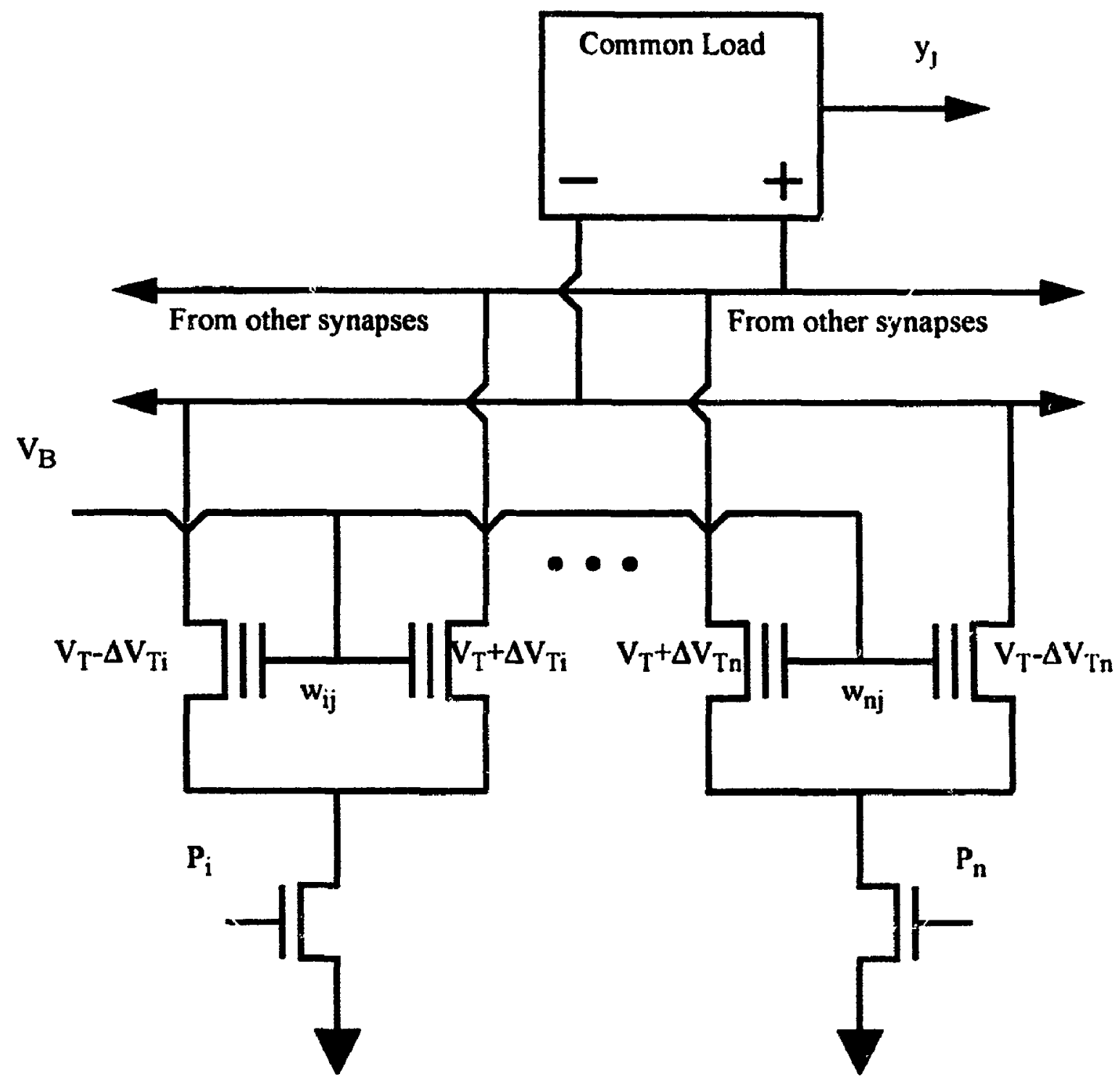

FIGURE 17 : Reduced-Transistor-Count Synapse

Different from the transconductance amplifier-based synapse implementation, the load transistors art shared between all synapses that input to a neuron. In this example, the weights are stored as the difference of the $V_{T} s$ in the differential pairs.

since the weight is stored as the difference in $V_{T} \mathrm{~s}$ between the two input transistors. Since this synapse design requires only three transistors, one bias voltage (which can be the same for all synapses), and no separate storage element, it is an excellent design choice. The floating gate voltage can be programmed to greater than 10 bits accuracy using standard techniques ${ }^{[21]}$. One technique is to apply a pulse voltage equivalent to the 
reverse-breakdown voltage between the well connection and the 'rain of one of the transistors of the pair. This causes avalanche breakdown, and reverse current flow, trapping charge in the transistor's gate by avalanche injection, altering the threshold voltage.

\subsection{Soma Circuits}

The soma completes the inner product circuit by summing the output of the synapses. In Section 4.7, several synapse circuits were examined, and the architecture of Figure 17 was determined to be an excellent implementation choice. Given the synapse architecture as a design constraint, the choices for good summation circuits are reduced. The summation circuit must sum a differential current input. Also, as will be seen in Section 4.9, simple methods for implementing the neuron activation function exist, which require a voltage input. The soma circuit must output a voltage signal. The use of a voltage signal as the output of the soma should not cause any loading problems, since the soma circuit can be located physically beside the activation function circuit, and the loading from the interconnect will be minimal. Since the soma circuit inputs only to a single activation function circuit, the fan-out load should also be quite reasonable.

Lont and Guggenbühl[ ${ }^{[19]}$ use a linear I-V converter similar to that shown in Figure 18. This design uses only seven transistors. This design differs from that of Lont's and Guggenbühl's in that the output of the I-V converter d not connect directly to the negative side of the differential synapse output, which reduces the voltage swing on the synapse output. Further, an asymmetry would result since the current of $M_{B}$ contributes to the current summation. These are not critical problems. 


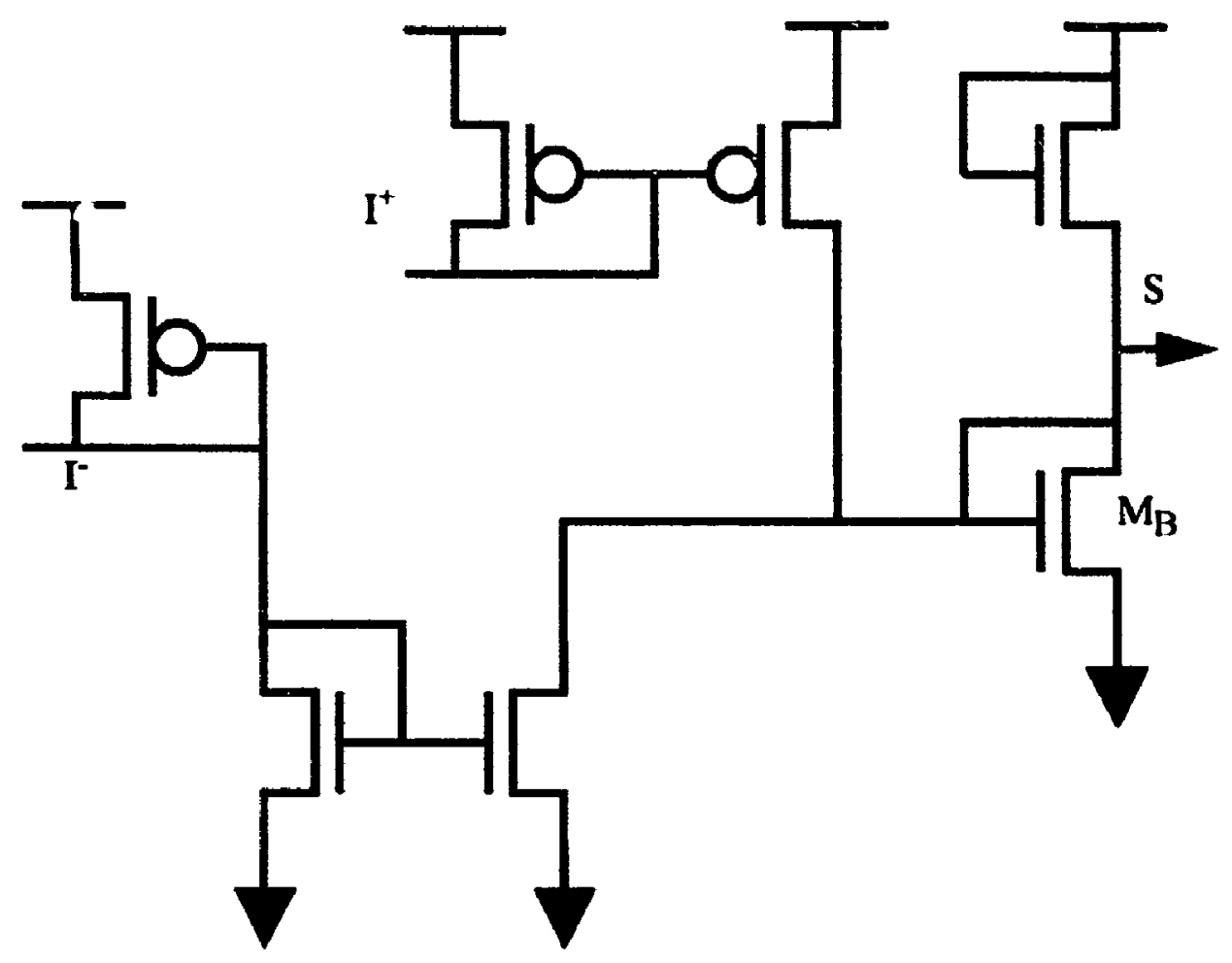

FIGURE 18 : Linear IV Converter

Sums the differential input current and outputs a voltage.

In another implementation, proposed by Bibyk and Ismail, ${ }^{[21]}$ the current thresholder of Figure 19 also sums the differential current input and outputs a single-ended voltage. The advantage of this circuit over the linear I-V converter is that it effecively isolates the output of the I-V converter from the synapse circuits. The use of an extra transistor is not critical, since the number of soma circuits used in the network is much smaller than the numbe: of synapses. The problem with this circuit, however, is that it limits the applications to those which use a binary threshold (a step function). 


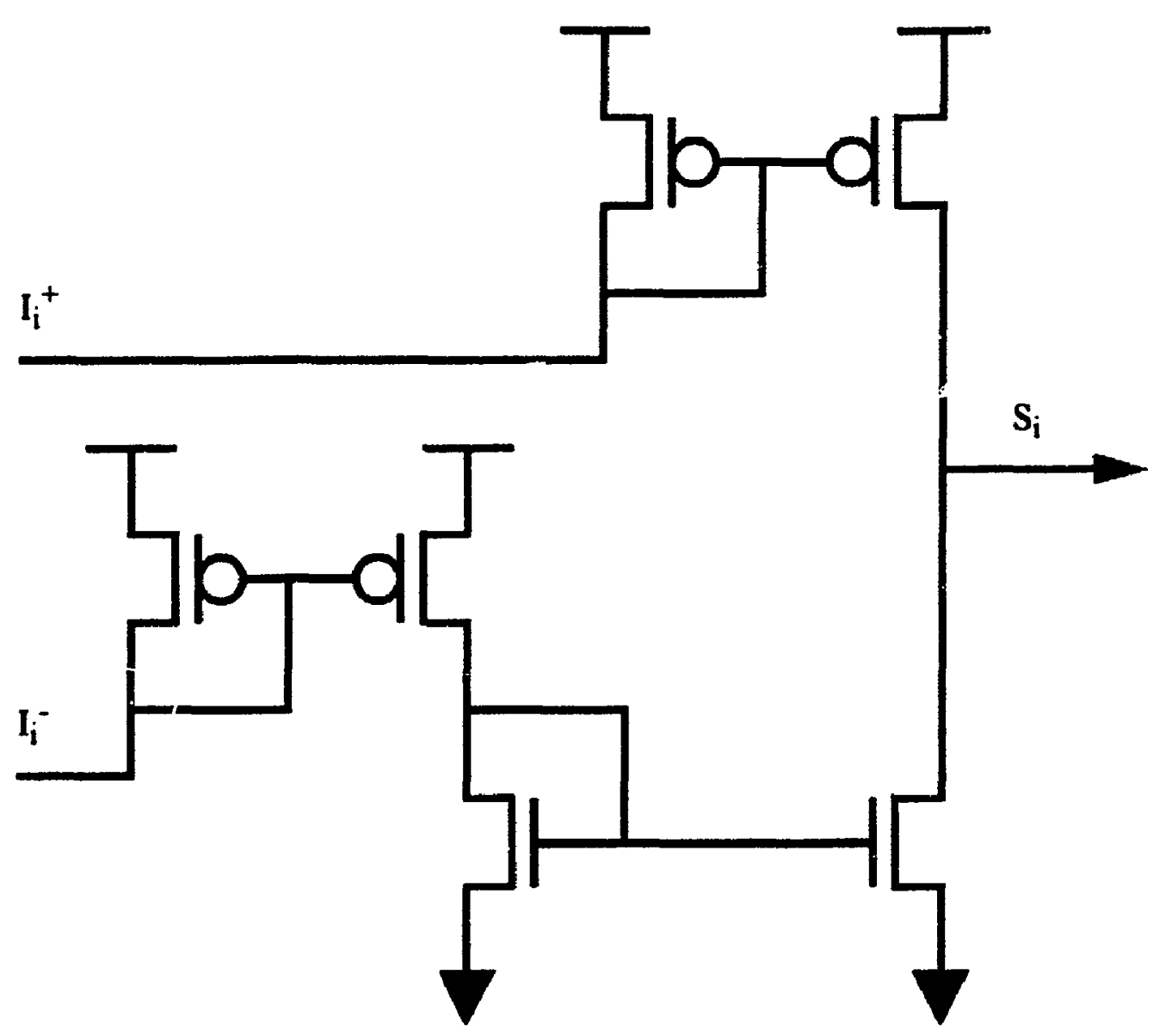

FIGURE 19 : Current Thresholder

Sums the differentiai input current and performs a current-to-voltage conversion. The output is 0 Volts when $I^{-}>I^{+}$and $V_{D D}$ when $I^{-}<I^{+}$.

\subsection{Analog Implementations of Activation Function}

The most common activation function implementation is presented in Figure 20 . The differential amplifier is an excellent choice for the activation function, since its transfer characteristic can be approximated accurately with the $\tanh \left(\right.$ ) function. ${ }^{[29]}$

Although five transistors are used to implement this function, area requirements are not critical in the activation function design. 


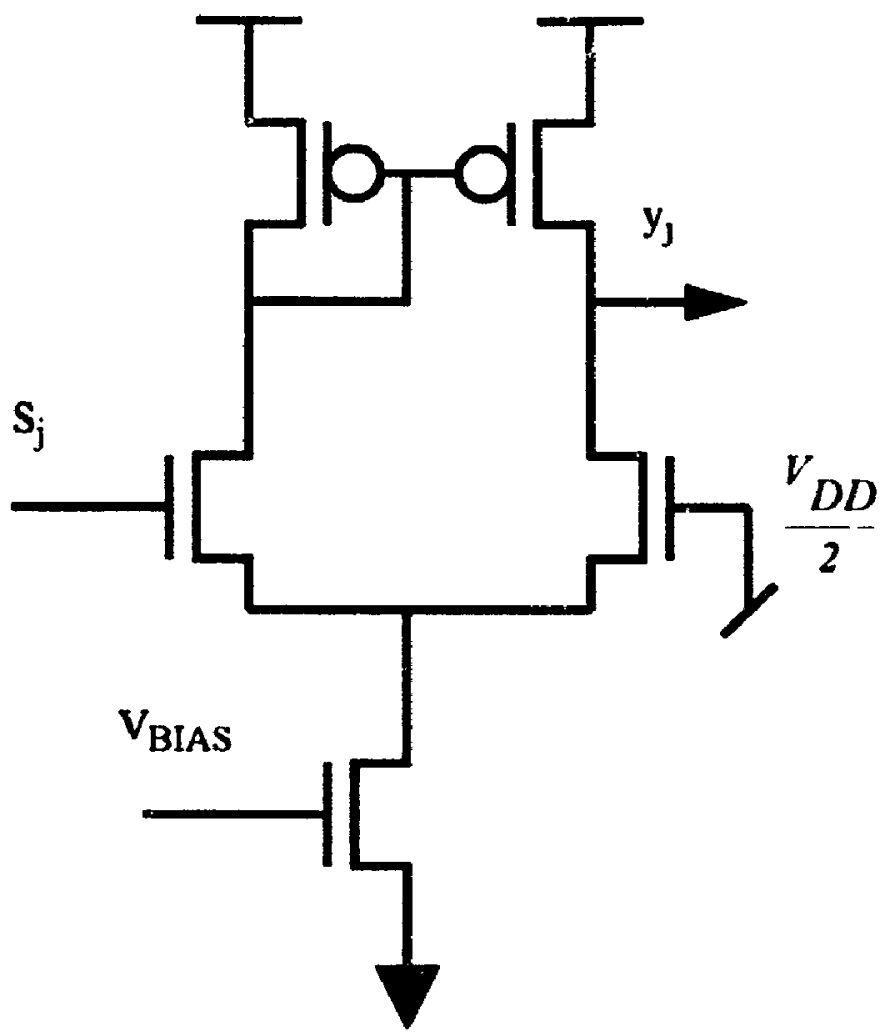

FIGURE 20 : Transconductance Amplifier-Based Activation Function

The transfer function of this circuit approximates a $\tanh ($ ) function.

Lee and Sheu ${ }^{[28]}$ implemented a cascode amplifier-based activation function. This activation function allows the implementation of annealing (a technique which simulates the gradual cooling of a system), which is sometimes used in backpropagation networks to speed learning. Although the use of a cascode can be avoided, the added complexity in implementing hardware annealing is not justified for cascade-correlation networks.

An alternate techniqu:e is to use just a regular CMOS inverter. ${ }^{[21]}$ The inverter is a desirable activation function because of its low area requirements.

A single analytical expression for the transfer function of the inverter cannot be derivec 


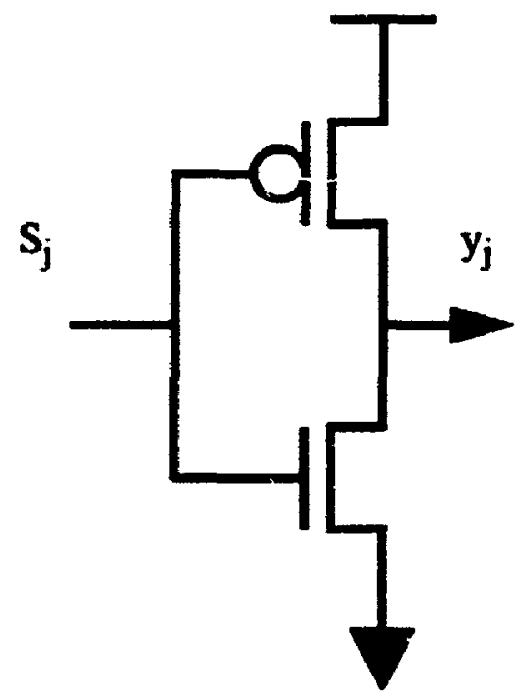

FIGURE 21 : CMOS Inverter-Baed Activation Function

This is the most simple implementation of an activation function. Unless a step activation furction is to be used, modelling of the transfer function for the learning algorithm makes the use of t':c CMOS inverter too computationally expensive.

using the standard transistor models, since for large voltage swing operation, the transistors do not remain saturated. As a result, several equations must be used to account for the different operating regimes. Models have been developed that allow a single equation to describe transistor response in bcth the linear and saturated regimes. These models are difficult to use, since they are non-polynomial equations and are not easily solvable. For example, Shousha's model ${ }^{[30]}$ is given by:

$$
I=I_{D D}\left(\frac{V_{G S}-V_{T}}{V_{D D}-V_{T}}\right)^{\alpha} \tanh \left(\frac{V_{D S}}{V_{D D}\left(\frac{V_{G S}-V_{\tau}}{2\left(V_{D D}-V_{T}\right)}\right)^{\alpha / 2}}\right) \quad, V_{G S} \geq V_{T},
$$

where $I_{D D}$ and $V_{D o}$ are the drain current and voltage at $V_{G S}=V_{D D}$ respectively, and $\alpha$ is the velocity saturation index, which ranges from two to one. Equation 28 can be solved for $V_{D S}$ only numerically, or by substituting a Taylor series expansion for the $\tanh ()$ function. The Taylor series expansion requires a minimum of six terms to achieve 12-bil precision, 
which results in unwieldy computations. Since this work assumes learning is to be accomplished on a host computer, and not in hardware, the computational complexity of the models is of paramount importance. The more complex the models are, the more computing resources are required to train the network.

Although the model for the transconductance amplifier (Section 5.4) is relatively complex, the amplifier can be designed such that all the transistors remain in one region of operation during the entire output swing, and an analytical expression can be obtained. Since, as previously stated, the area requirements of the activation function are not critical, the transconductance amplifier is an adequate choice to implement the activation function.

\subsection{Chapter Summary}

In this chapter, a number of implementations technologies were evaluated for their suitability to the implementation of ANNs. An implementation based on CMOS technology, at the present state of technology, was found to be best choice. Of the two broad design styles available in CMOS, digital and analog circuitry, analog circuitry was found to be more suitable, since it offers a more cost-effective solution for the implementation of basic neural network components.

Many different design options are available for the implementation of the basic components required to build ANNs. Some of the more popular implementation schemes were discussed. It was found that a differential pair-based multiplier incorporating floating gate transistors in the pair to accomplish weight storage as the difference in the programmed $V_{T} \mathrm{~S}$ of the two transistors is a good choice for the synapse implementation. 
This structure uses a load that is shared among all the different synapses inputting to a neuron. Given this synapse implementation, the logical circuit for the neuron body is the linear I-V converter. A good choice for implementing the activation function is a transconductance amplifier, since it can be easily modelled (Section 4.9).

The sub-circuit implementations chosen here will be discussed in more detail in the following Chapter. Models of these circuits will be developed allowing for the verification of the learning algorithm presented in Chapter 6. 


\section{Chapter 5. Analog Neural Network}

\subsection{Introduction}

The architecture of Figure 22 is analyzed in more detail in this section. This architecture is similar to the one proposed by Bibyk and Ismail, ${ }^{[21]}$ except for the activation function. Bibyk and Ismail use an inverter that implements a $\operatorname{sgn}($ ) function as the activation function. The architecture proposed in this work was chosen for a number of reasons, as discussed in Chapter 4, including the ease for which models can be developed for each of the subcircuits.

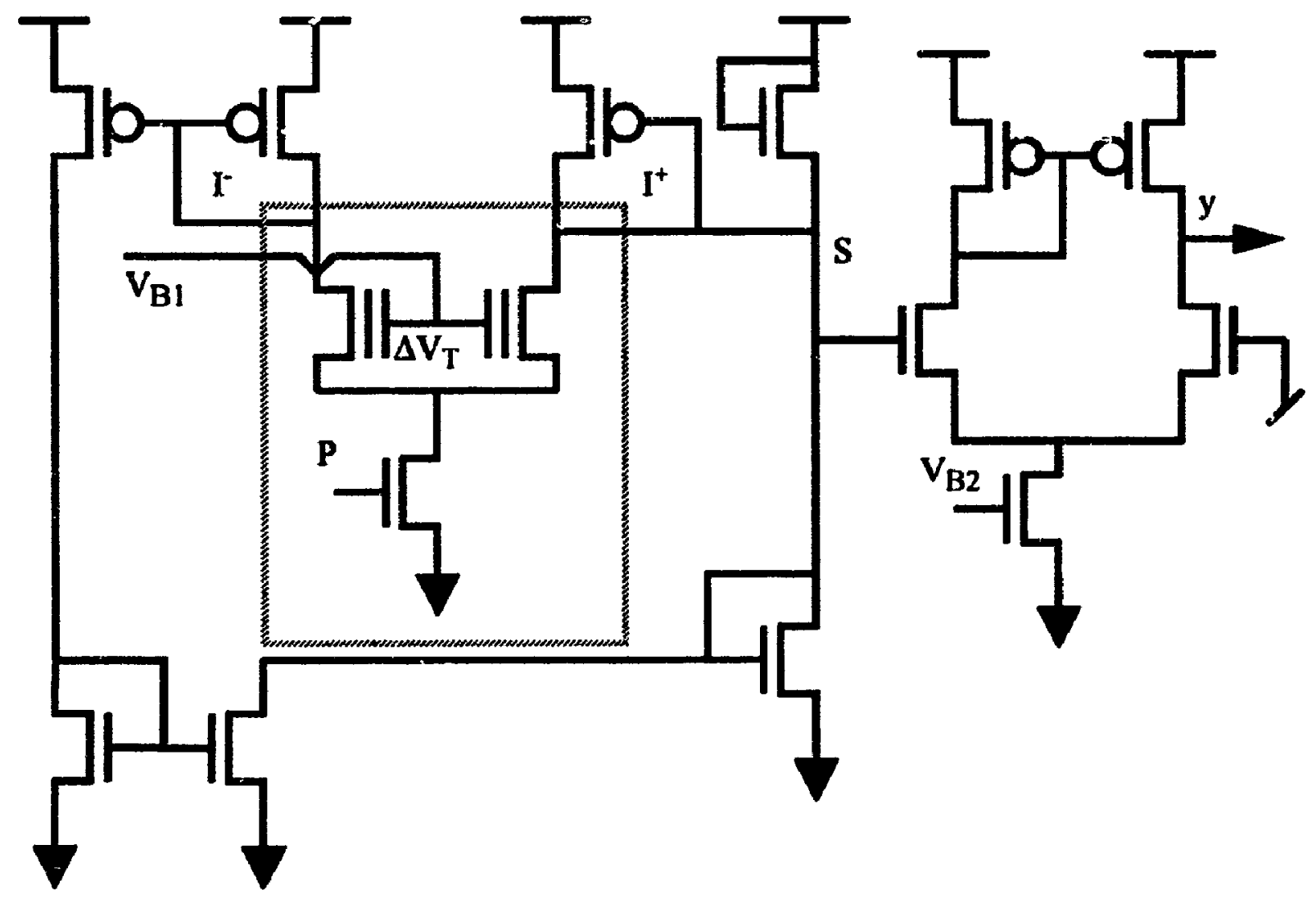

FIGURE 22 : Neuron-Synapse Structure

Only one representative synapse is shown. In a real network, many synapses would be connected in parallel, sharing the same $V_{B l}$ and having independent $P \mathrm{~S}$ and programmed thresholds. 
The ideal neuron transfer function cannot be simply implemented using analog circuitry. Today, researchers use the standard neuron equations to develop the training algorithm, then create circuits to model these equations as closely as possible. This technique limits the trainability of the network, since the neuron transfer function implemented on the IC does not match the transfer function implemented in the learning algorithm. The approximation typically holds until network error drops below about 10 percent. At this point, the differences between the integrated network behavior and the bulavior expected by the training algorithm implemented on a computer become more critical.

In this section, the actual transfer function of the circuit is obtained using SPICE simulations. Based on this transfer function, a new learning algorithm is proposed in Chapter 6. Since the learning algurithm is developed based on the circuit equations, the mismatch between the error exhibited by the network and the leaming algorithm during training should be reduced.

\subsubsection{Motivation}

The entire network does not need to be simulated to obtain the circuit equations for the learning algorithm. This technique requires that only a single neuron and synapse be simulated for obtaining the transfer functions. ${ }^{1}$ Once the transfer functions have been obtained, any number of approximation techniques can be used to minimize the difference between the equations used in the learning algorithm and actual circuit performance, depending on the degree of accuracy required.

1. Further simulations will be necessary to ensure the accurate performance of the manufactured circuit under all operating conditions. These simulations are only loosely related to the simulations being discussed here. 
These approximations are necessary. Early research into the design of neural networks in VLSI erroneously assumed that the network can compensate for transistor parameter variations. Although this is tias to some extent, these variations become quite critical as network size increases, and/or during the final stages of training. Many researchers analyze these non-ideal effects for the sole purpose of determining the feasibility of the target subcircuit design and network size, as in Choi et. al.. ${ }^{[31]}$ among others. Any variations in the execution of the neural transfer function are treated as non-ideal effects. These effects combine with the non-ideal transfer functions of the implemented subcircuits to produce a neural network that cannot be easily trained. Since transistor parameter variations can be predicted only in a statistical manner, it is impractical to compensate for these variations precisely. By separating the two causes of untrainability in VLSI neural networks, and minimizing one of them, the resulting neural network might display an improved leaming ability.

It is critical that all non-ideal effects be fully understood before a practical ANN can be implemented. However, this work proposes that these effects need not be considered as limiting factors to network performance. If the effects are wall understood, and analytically expressed, they can be incorporated to create a s,ecialized neuron transfer function that is specific to the design. Once these effects are incorporated into the transfer function, they can then be ircerporated into the learning algorithm, thus allowing a larger network with simpler units to be impleniented and trained.

In this chapter, simple models of non-ideal effects are presented, allowing the development of a modified neurai transfer function, which is used to help illustrate the 
modified learning algorithm presented in Chapter 6.

\subsection{Synapse}

As discussed in Chapter 4, a gud implementation choice for synapses is given by a differential pair designed using floating gates (repeated in Figure 23). This circuit is different from standard nultipliers because it multiplies the input signal by the difference in the $V_{r} s$ of the differential pair, rather than by a bias voltage. The use of the floating gate transistors allows the synapse weight to be implemented in a non-volatile manner as the difference in $V_{T} s$ of the input pair. This further reduces complexity, as compared to a standard differential pair-based synapse, since the weight does not require a separate storage capacitor.

The chosen configuration uses two floating gates in the differential pair. Since the synapse value is determined by the difference in $V_{T} \mathrm{~s}$ it cculd be argued that only one floating gate

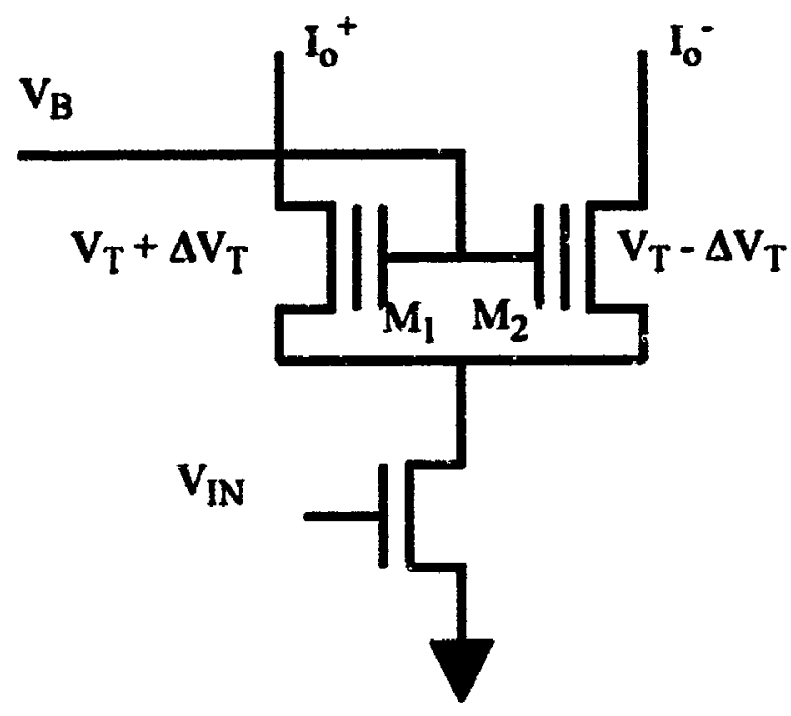

FIGURE 23 : Floating-Gate, Differential Pair-Based Synapse 
is necessary, and the second transistor can be a standard NMOS transistor - as shown in Figure 24. The configuration of Figure 24, however, dnes not allow for good matching between the two input iransistors, resulting in an amplification of the contribution of transistor mismatch between the two transistors in the differential pair. For this reason, it will not be considered.

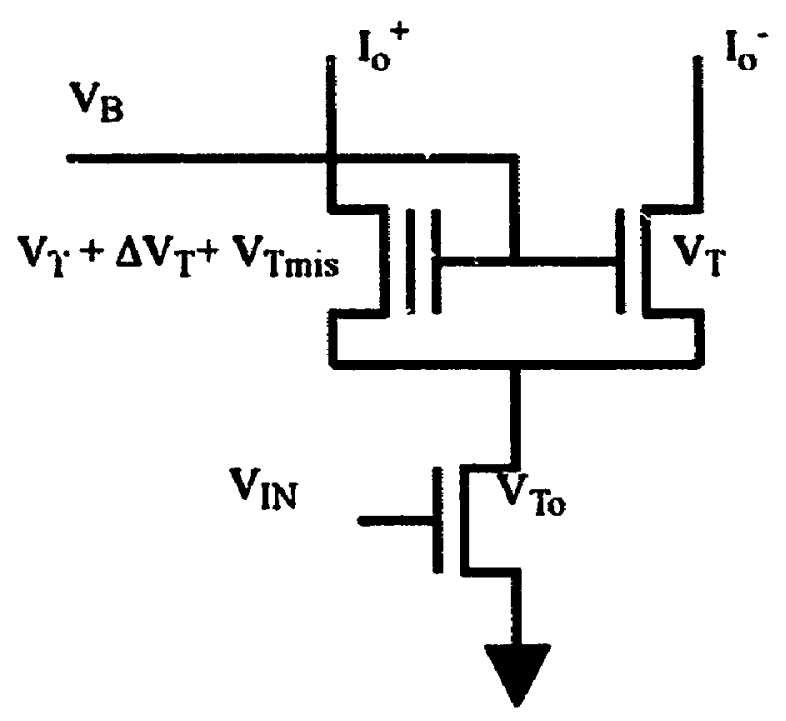

FIGURE 24 : Simpler Synapse Configuration

The configuration $f$ Figure 23 produces a differential current that approximates the multiplication of $\Delta V_{T}$ and the input voltage, $V_{I N}$. Analysis of this circuit was not found is the literature, and is presented below.

Since the inputs to the differential pair are always at the same potential, the differencemode input voltage is always zero. Assuming the input transistors are saturated and 
matched - except for their $V_{T} \mathrm{~s}$ - the current through the inpui transistors is written as:

$$
\begin{gathered}
I_{M 1}=\frac{\beta}{2}\left[V_{G S_{1}}-\left(V_{T}-\frac{\Delta V_{T}}{2}\right)\right]^{2}, \quad I_{M 2}=\frac{\beta}{2}\left[V_{G S_{2}}-\left(V_{T}+\frac{\Delta V_{T}}{2}\right)\right]^{2}, \\
\text { where } V_{T}=\frac{V_{T 1}-V_{T 2}}{2}
\end{gathered}
$$

Since $V_{G S 1} \approx V_{G S 2}$, solving the equations for $I_{M 1}$ and $I_{M 2}$ for $\Delta V_{T}$ and adding yields:

$$
\Delta V_{T}=-\sqrt{\frac{2}{\beta}}\left(I_{M 1}-I_{M 2}\right),
$$

recognizing,

$$
I_{s s}=I_{M 1}+I_{M 2}
$$

allows $I_{M I}$ to be solved in terms of $I_{S S}$ and $\Delta V_{T}$. Substituting Equation 32 into Equation 31 results in:

$$
\Delta V_{T}=\sqrt{\frac{2}{\beta}}\left(I_{s s}-I_{M 1}\right)-\sqrt{\frac{2}{\beta}} I_{M 1} .
$$

Squaring twice and solving the resulting quadratic in $I_{M l}$ gives:

$$
I_{M 1}=\frac{I_{s s}}{2}+\frac{\beta}{2} \Delta V_{r} \sqrt{\frac{4 I_{s s}}{\beta}-\Delta I_{T}^{2}} \quad \text { and } \quad I_{M 2}=\frac{I_{s s}}{2}-\frac{\beta}{2} \Delta V_{T \sqrt{ }} \sqrt{\frac{4 I_{s s}}{\beta}-\Delta V_{T}^{2}}
$$

For a seural network using a learning algorithm based on the standard synapse transfe function, this circuit can be used only as an approximation, since as $\Delta V_{T}$ grows significant compared to $4 I_{S S} \beta$, the approximation to a true multiply tecreases. Assuming $\Delta V_{T}$ is 
small compared to $4 I_{S S^{\prime}} \beta$ (which can be a design constraint):

$$
I_{o}=I_{M 1}-I_{M 2}=\beta \Delta I_{T_{1}} \sqrt{\frac{4 I_{s s}}{\beta}}=\sqrt{2} \beta \dot{\Delta} l_{r}\left(l_{i V}-r_{r_{o}}\right)
$$

and there is a multiplication between $\Delta V_{T}$ and $V_{I N}$, as required.

Without making this approximation,

$$
l_{o}=\beta \Delta V_{T} \sqrt{2\left(V_{I N}-V_{T o}\right)^{2}-\Delta V_{T}^{2}} .
$$

The above derivation uses the simple Shichman and Hodges model for the MOS transistors. Although this is a fairly severe approximation, it has two important merits. Firstly, Equation 36 more closely models the actual behavior of the synapse than the assumption that the synapse implements a true multiplication. Secondly, the computational complexity of Equation 35 is low compared to an accurate SPICE simulation. A more accurate model of the circuit behavior can only be accomplished at the expense of more complicated transistor models - but would still result in less complex models than those used by SPICE. Since the learning is typically implemented on a general-purpose computer, the computational complexity is of primary importance.

A simulation result for the synapse is shown in Figure 25. 


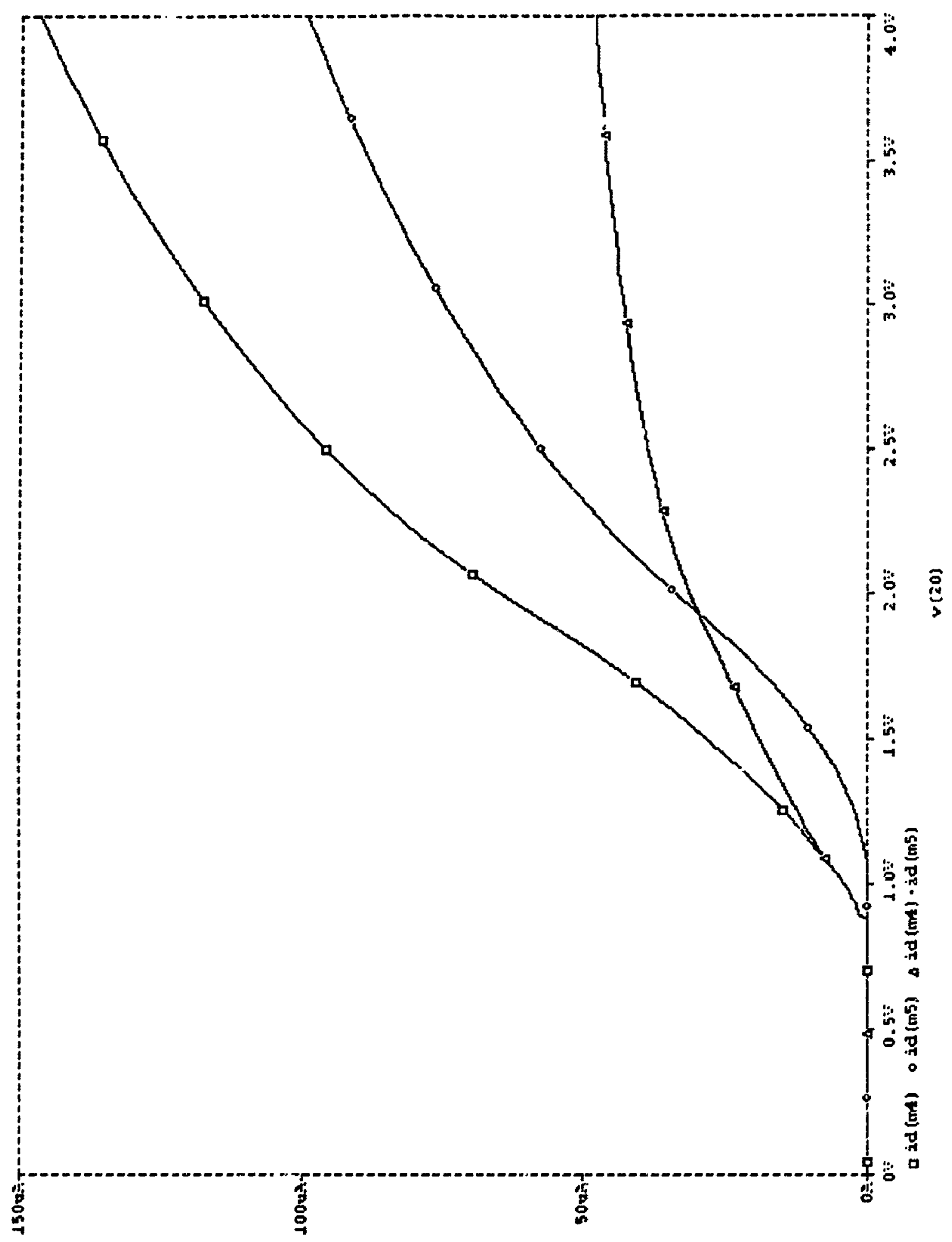

FIGURE 25 : Synapse Simulation

$I D(m 4)$ and $I D(m 5)$ represint the output currents of the synapse as a function of the input voltage $(v(20 ;)$ $I D(m 4)-I D(m 5)$ displays the multiplication ope ' tion of the synapse. 


\subsection{Neuron Body}

The neuron body sums the output of each input synapse, thus completing the innerproduct circuit.

Equation 34 is the output of a single synapse. The output currents $I_{M I}$ and $I_{M 2}$ (Figure 23) can be summed with those of the other synapses using Kirchoff's current law on lines I' and $\mathrm{I}^{*}$. If $N_{S}$ synapses are connected to a neuron, then the sums become:

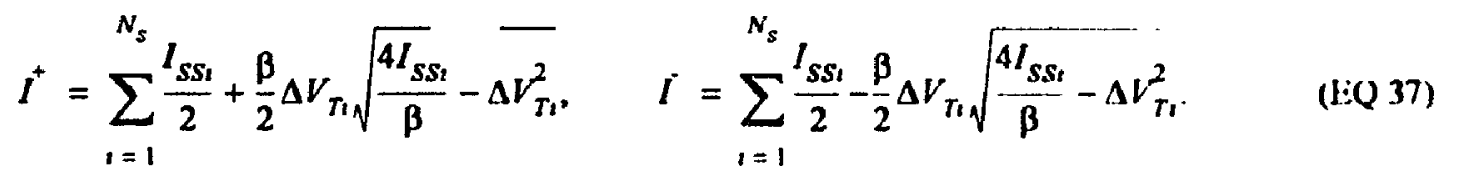

Each half of the differential outputs have been summed. To combine these signals into a single-ended output, the linear surrent-to-voltage converter discussed in Section 4.4 and repeated in Figure 26 is used.

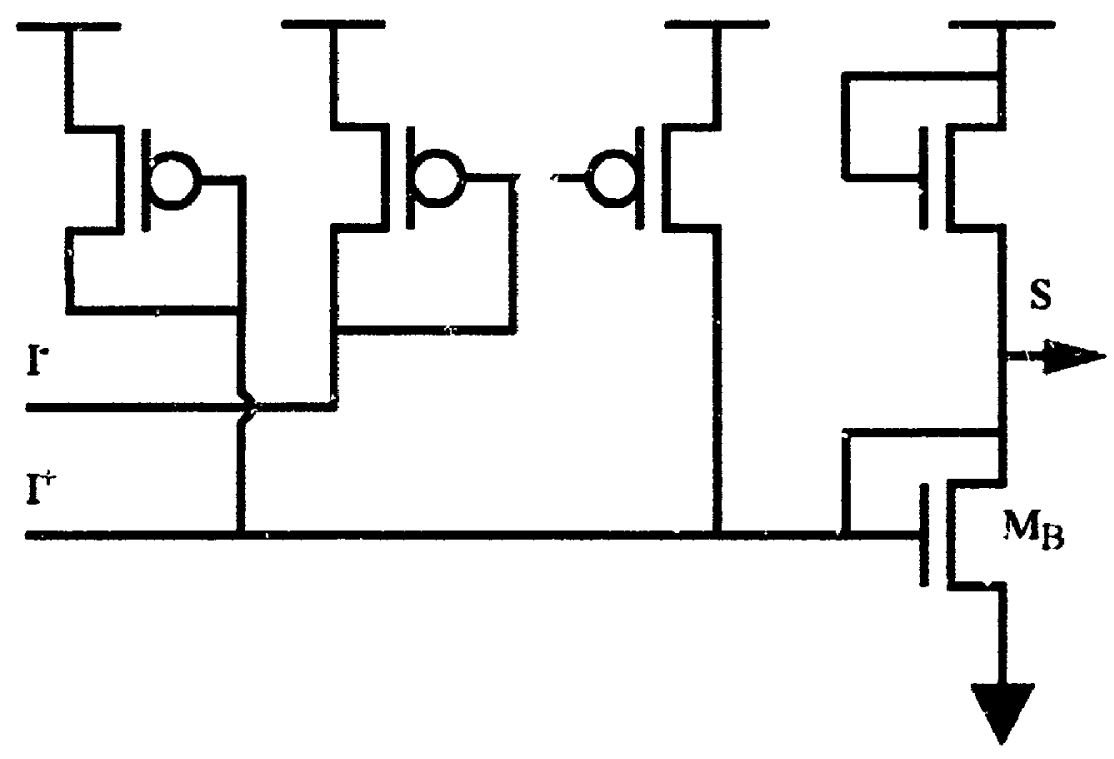

FIGURI; 26 : Linear Current-to-Voltage Converter 
Since the number of neurons is much smaller than the number of synapses, and only one current-to-voltage converter is required per neuron, transistor area is not a critical factor for this circuit.

The current mirror is used to convert the summed differential output current of the synapse to a single ended current. The two diodes act as a simple resistor divider, converting this current to a voltage. A simulation of the current-to-voltage converter which demonstrates the good linearity of this circuit is presented in Figure 27. 


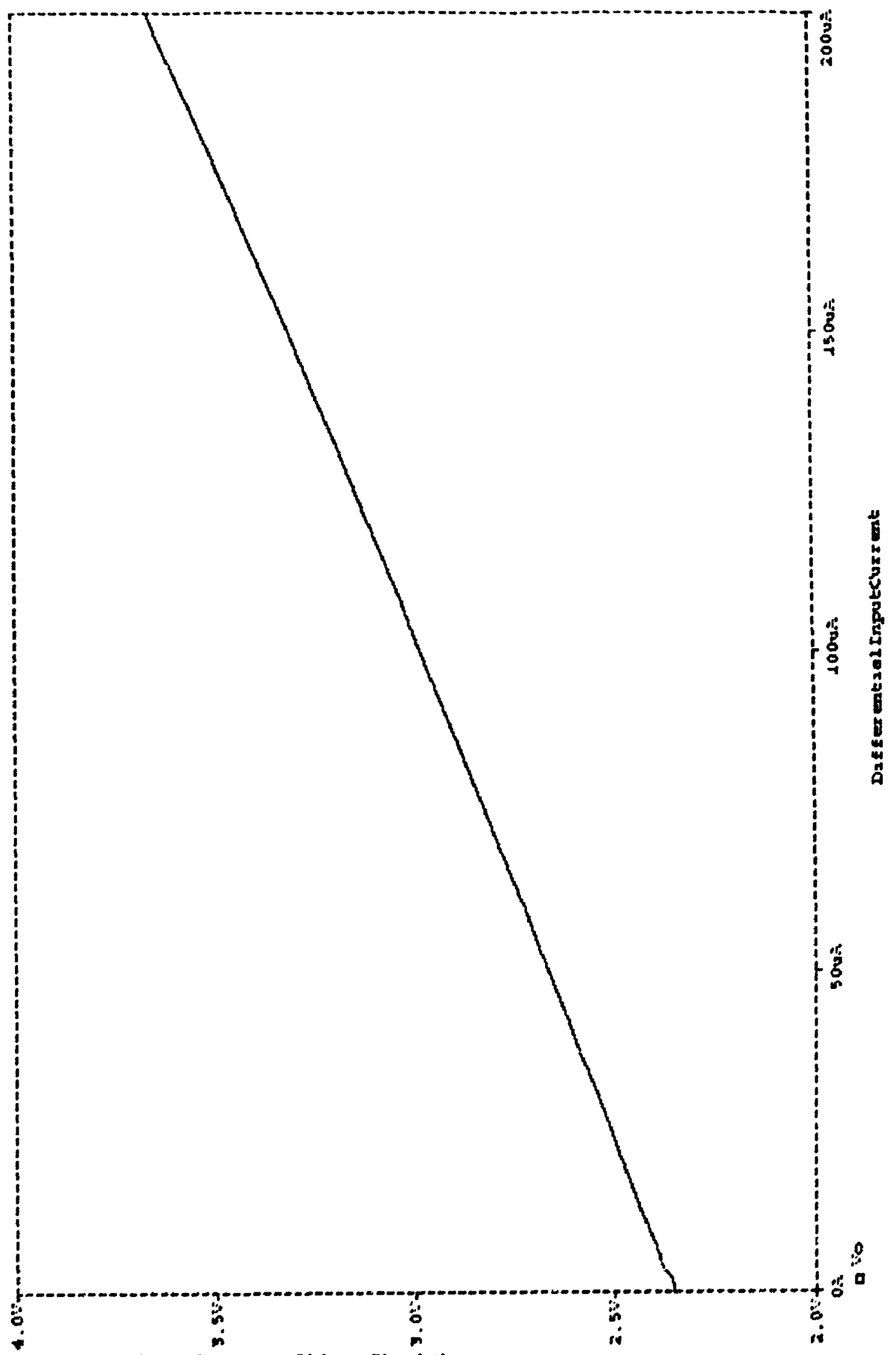

FIGURE 27 : Linear Current-to-Voltuge Simulation

Linear response of the linear current-to-voltage converter of Figure 26. 


\subsection{Activation Function}

The simplest implementation choice for the activation function is a CMOS inverter. As discussed in Section 4.4, since the transistors in a CMOS inverter pass through both the linear and saturation regions during a transition, a simple analytical expression cannot be formed. An alternative is to use different circuit techniques, or to limit the input to the inverter so that the transistors remain saturated, and an analytical expression can be derived. Limiting the input range is not a viable solution, since the sigmoid-like shape is lost. As considered in Section 3.3, a better solution would be to use device models that require only one expression for both regions of operation. Unfortunately, these lead to unwieldy equations, so it is not be considered here.

Using the amplifier of Figure 28, a $\tanh ($-like function can be designed.

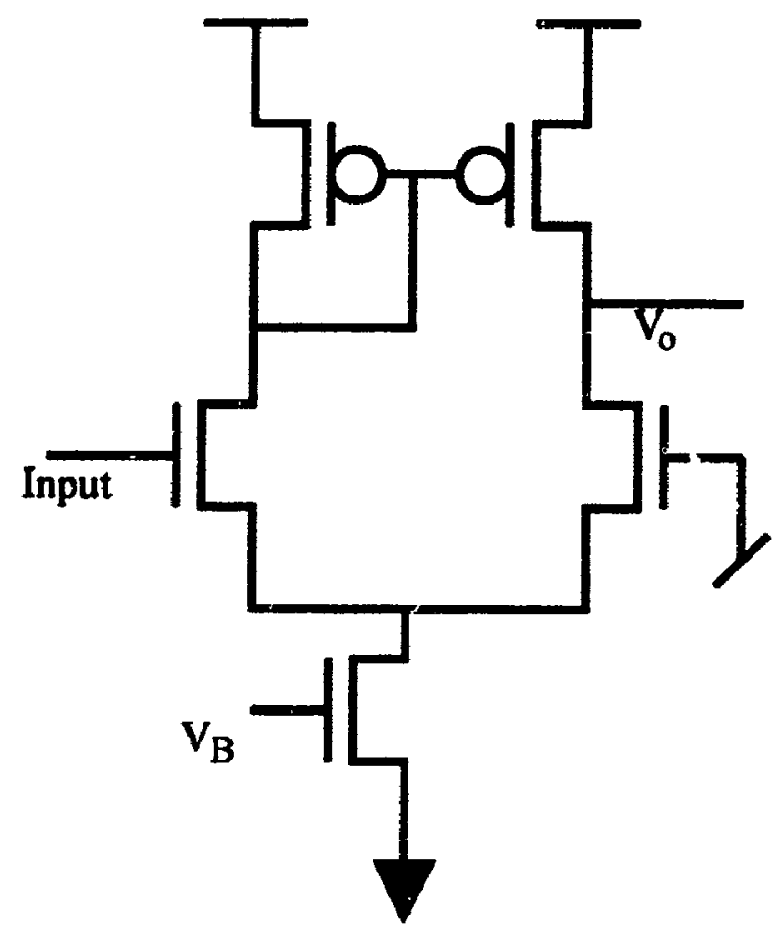

FIGURE 28 : Transconductance Amplifier Activation Function

The bias voltage. $V_{B}$ can be chosen such that it is $V_{D D^{\prime}} 2$, reducing the number of necessary bias voltages to one. 
The transfer characteristic of Figure 28 is available from many textbooks, including Allan and Holberg, ${ }^{[27]}$ and is simply repeated here as:

$$
V_{o}=I_{s s} \sqrt{\frac{\left(\beta v_{D}^{2}\right)}{I_{s s}}-\frac{\beta^{2} l_{D}^{4}}{4 I_{s s}^{2}} R_{L}}
$$

The transfer function of this circuit is sigmoidal in shape, as is apparent from the simulation result included in Figure 29. Although it not a true sigmoid in shape, it is differentiable, and has all the same properties as the sigmoid. It is an equivalent transfer function.

\subsection{Chapter Summary}

Using the results of the feasibility study presented in Chapter 4, a circuit architecture was chosen which is suitable for the implementation of ANNs in VLSI. Simulations were performed on these circuits to verify that they actually do perform the required functions. Models were developed for each of the components used to implement the neural network. These models can now be used in a neural network training algorithm (Chapter 6) specifically designed for training a neural network implemented in VLSI using these circuits. 


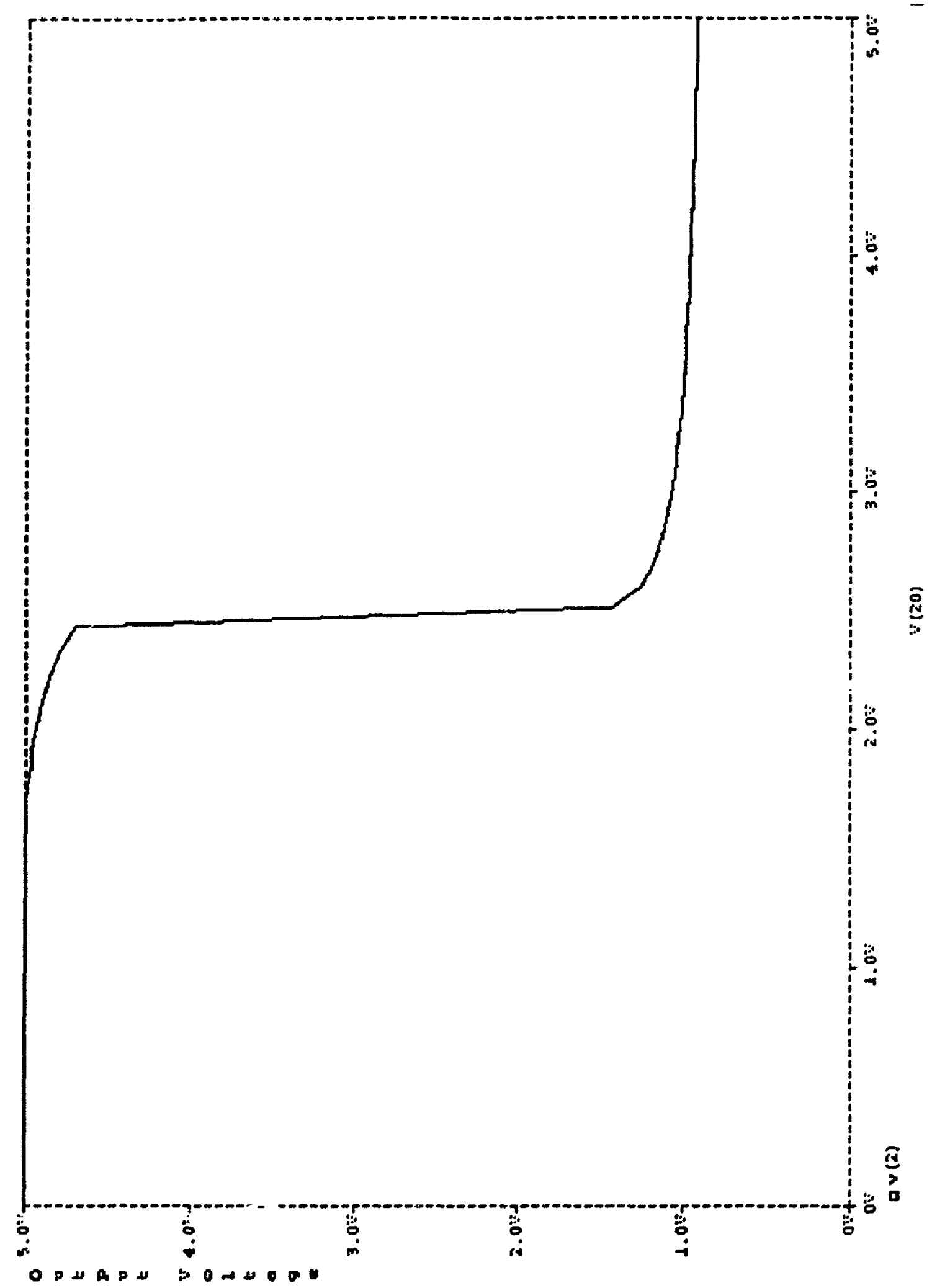

FIGURE 29 : Activation Function Simulation Result

$v(20)$ is the input voltage. 


\section{Chapter 6. Customized Learning}

\subsection{Introduction}

As discussed in Chapter 4, the transfer functions of neurons and synapses are known to arbitrary precision when the neural network is modelled in software. This luxury is not available in integrated VLSl implerientation:. Digital implementations suffer from a loss of numerical precision, and analog implementations display approximatiors to the functions being implemented. As a result, algorithms that display adequate learning properties in software do not function well when mapped to a VLSI architecture.

A number of different techniques have been developed to overcome this problem. $\mathrm{Tl}:$ : most common technique is to do a coarse and a fine training. First, a network with the same topology as the VLSI ANN is trained on a general purpose computer. The trained weights are downloaded to the VLSI network, and the VLSI network is trained using these weight values. ${ }^{[19]}$ This technique increases the chances of convergence when training the VLSI network. It does, however, increase the computational cost. Since weight updates are normally calculated off-chip, the network is effectively trained twice. Further, even though nowinally valued weights have been loaded, the network will noi perform as well as the software version because the actual neuron and synapse transfer functions of the VLSI network differ from the transfer functions assumed by the leaming algorithm Results presented by many researchers, including Lont, ${ }^{[19]}$ have shown that analog networks learn quite admirably until the error value becomes relatively smali. At this point, the differences between the transfer functions implemented in the training algorithm and $0: 1$ silicon become very significant, and no further improvement in the error value can 
be achieved.

Modifying existing training algorithms to increase the performance of VLSI implemented neural networks is a common technique. Caviglia ${ }^{[32]}$ and Lont ${ }^{[19]}$ present two different modifications to the backpropagation algorithm. In Caviglia's paper, a variant to backpropagation is presented that allows discretely valued weights. The modification to the algorithm addresses the problems backpropagation has in dealing with non-continuous weight values. Lont's paper modifies the basic algorithm to handle non-linear synapses. Both these algorithms alter the original backpropagation algorithm to incorporate one specific aspect of the VLSI neural network that deviates from the ideal architecture defined by the algorithm.

To ensure maximum generalization abilities and learning properties, the actual transfer functions of all the VLSI ANN components must be incorporated. To determine the transfer functions precisely, transistor-level SPICE-like simulations are necessary. Due to the high computaticnal requirements of SPICE simulations, it is not feasible to incorporate SPICE models into a learning algorithm for training the network. Typically, the VLSIimplemented ANN is assumed to contain neurons/synapses which behave ideally, which can be a severe approximation. This is one of the factors which contributes to the degraded performance of VLSI neural networks.

The second factor which contributes to the degraded pertormance of VLSI neural networks is device non-idealities. These non-idealities, caused by variations during manufacture, cannot be predicted, and cannot be compensated for withcus device characterization. Correction for this type of degradation is not feasible. 
To minimize the effects of the severe approximations to the neuron transfer function, simple models - as compared to the SPICE models - will be incorporated into the learning algorithm.

Recalling the standard neuron transfer function:

$$
y_{\jmath}=f\left(\sum_{1} p_{1} w_{v j}+\theta_{\jmath}\right) .
$$

three major functions are present: the activation function, the synapse multiplication, and the soma summation. As noted in Chapter 2, the threshold can be incorporated into the summation, and thus does need to be explicitly considered. Each of these three functions are implemented separately in the VLSI network as discussed in Chapter 4, and can therefore be addressed individually.

The network of Chapter 5 implements the activation function using a transconductance momplifier, whose transfer function is given in Section 5.4.

The transfer function of the transconductance amplifier, like the traditional sigmoid, is non-linear. As a result, the modified activation function does not alter the learning algorithm except in its final application, when the modified activation function is used instead of the traditional leaming function. The sigmoid and the tanh() functions are popular because of their convenience - the sigmoid for its easily calculable derivative; the $\operatorname{tanh()}$ for its ease of implementation. Since the transconductance amplifier activation function possesses the same shape and properties, the learning properties of the network will not be adversely effected by this replacement, and the modifications to the leaming 
algorithm are trivial. Additionally, if different models vere used, a $\tanh ()$ description can be derived.

The summation uses a differential style of design. The positive and negative currents are added using the linear current-to-voltage converter, which provides an input voltage to the activation function circuit (the transconductance amplifier). The summation accomplished by the circuit of Chapter 5 actually converts the differential input current to an output voltage in a linear fashion. If the summation is replaced with a non-linear function, the operation ceases to be associative, and a closed-form derivation of the learning algorithm becomes quite difficult. One method to reduce complexity is to isolate the linear and nonlinear operations:

$$
y=g\left(\beta_{a} \sum_{j} I_{j}^{+}+\beta_{b} \sum_{k} I_{k}\right)=g\left(\sum_{,}\left(\beta_{a} I_{1}^{+}+\beta_{b} I_{j}\right)\right) .
$$

Here, $g$ represents the non-linear portion of the operation, and the $\beta$ s represent any mismatch in the gain between the positive and negative sides of the current paths. In this example, $g$ could be lumped with the activation function, leaving a linear summationi operation.

Finally, the actual weight storage and synapse multiplication, most generally, are ...unlinear due to the non-linearity of the synaptic storage element. Since these effects can be characterized - either through analytical or empirical methods - these operations can also be incorporated into the software modei. 
A top level description of the nəuron transfer function becomes:

$$
y_{1}=f\left(\sum_{j} h\left(y_{1}, w_{10}\right)\right)
$$

where $f$ still signifies the activation function, and $h$ represents the nonlinear synapse operation.

In Chapter 3, the cascade-correlation algorithm was introduced and argued to be better suited than backpropagation for the training of VLSI-implemented neural networks. Since a new, top-level neuron transfer function containing non-linear synapses has been introduced, the cascade-correlation algorithm must be re-derived to accommodate the new transficr function.

Further, the standard cascadc-correlation algorithm is based on continuous weight values. Although the weights are stored in an analog fashion, their actual value can only be updated to a finite precision. Consequently, to improve the learning characteristics of the network, the effects of this limited numerical precision should be included. Hoehfeld and Fahlman ${ }^{[1]}$ have already performed a detailed study of these effects, and have proposed a solution that allows the network to be trained on problems requiring even very steep discriminating boundaries, even as the precision of the weights, sigmoid, and weightupdates are simultaneously restricted to only seven bits using a modified cascadecorrelation algorithm. Their results are incorporated into the new algorithm presented in Section 6.2. 




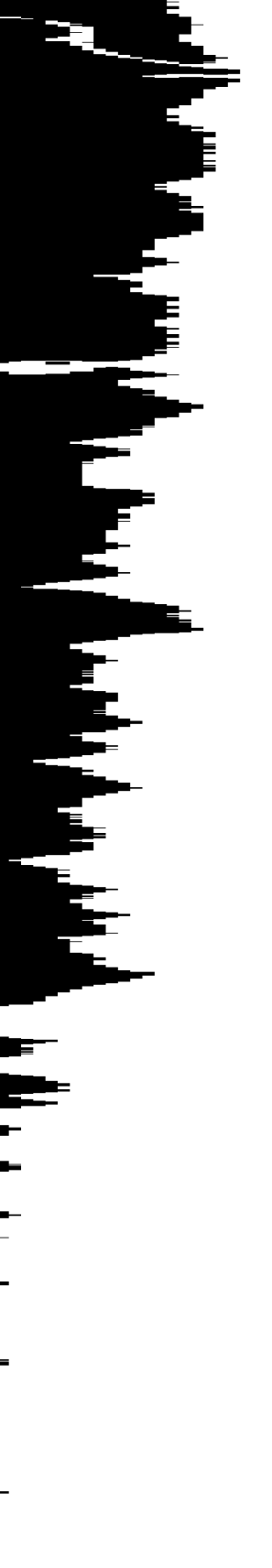






$$
2 \text { of } / \mathrm{de} 2
$$




\subsection{VLSI Cascade-Correlation}

No published work is available on a modified cascade-correlation algorithm being implemented for VLSI. However, Lont and Guggenbühl ${ }^{[19]}$ have a modified backpropagation for use with VLSI implementations containing non-linear synapses. Their results are encouraging, illustrating that although the implemented VLSI network cannot be trained using the standard backpropagation algorithm, it can learn with the modified backpropagation algorithm if a weight set developed from the simulator is used as the training starting point. Their lack of success training the network from an initial random weight set can be partially attributed to the fact that they did not consider the reduced numerical precision of their synapses, as compared to the high precision assumed by the backpropagation algorithm.

Applying Hoehfeld's and Fahlman's work in conjunction with Lont and Guggenbühl's, and the results of the previous chapters, can produce a robust VLSI training algorithm and network.

The effects of limited numerical precision can affect every aspect of the neural network The summation and multiplication, the sigmoid calculation, the weight storage, and the weight update can each have different levels of accuracy. It his already been shown that the summation will not deviate far from the ideal. The multiplication operation is inherently non-linear, but is a continuous function, and can be treated as being quite precise (assuming the non-linearities are incorporated in the learning algorithm). The sigmoid calculation, implemented in an analog manner, also does not suffer from any lack of numerical precision. The actual weight storage and the weight update are inherently 
linked. The accuracy of the stored weight value depends on the accuracy of the weight update. Since the weights are stored in an analog manner, the only numerical precision limitation when using the network of Figure 22 is the weight update, which can be accomplished only to a finite precision.

Following Hoehfeld and Fahlman's work, ${ }^{[11]}$ limited precision operations can be represented using a precision function, $P(x, n)$, defined by:

$$
P(x, n)=\left\{\begin{array}{cl}
\min & x<\min \\
\max \left(1-2^{-(n-1)}\right) & x>\max
\end{array},\right.
$$

when $x$ is either below or above the minimum and maximum values permitted by the operation. When $x>\min$ and $x<\max , x$ is rounded down to the nearest power $2^{-\mathrm{n}-1}$. The first two cases handle situations where $x$ is beyond the allowable range, while the third case quantizes legitimate values of $x$ to $n$ bits of precision.

Since the only place limited numerical precision effects the cascade-correlation algorithm (for the architecture of Chapter 5) is the weight update, the only change to the prototype cascade-correlation algorithm that is imposed is the modification of Equation 18 to:

$$
w_{t+1}=w_{t}+P\left(\Delta w_{p} n\right)
$$

This is a much simpler result than the results presented by Hoehfeld and Fahlman. In their work, they simultaneously limited the numerical precision of every element in the network. Assuming network values can be read off-chip for training with arbitrary precision, the only place that precision is limited is in the weight update itself - the standard algorithm can be used to calculate the weight update, using full precision, and 
quantized just before updating. Quantizing the weight update automatically quantizes the weight value. ${ }^{1}$

To incorporate the exiects of the non-linear synapse multiplication, the core of the cascade-correlation algorithm must be re-derived so the new expressions for $\frac{\partial E}{\partial w}$ and $\frac{\partial w}{\partial w}$ can be developed. The actual expression for $\Delta \mathrm{w}$ (given by Equation 19 and Equation 20) remains unchanged, as it does not explicitly depend on the weight value.

Recall the J.MS Error:

$$
E=\frac{1}{2} \sum_{j=1}^{N_{o}}\left(y_{j}-d_{j}\right)^{2}
$$

To minimize the LMS error, $\frac{\partial E}{\partial w}$ must be known. To calculate $\frac{\partial E}{\partial w}$, the chain rule is used, such that:

$$
\frac{\partial E}{\partial w}=\frac{\partial E \partial y}{\partial y} \frac{\partial E}{\partial w}=\frac{\partial y}{\partial y} \frac{\partial g}{\partial g}
$$

where $g$ is the output frorin the non-linear synapse. All these derivatives are known: $\begin{aligned} & \partial E \\ & \partial y\end{aligned}$ is simply calculated; $\frac{\partial y}{\partial g}$ is just $g$, and $\frac{\partial g}{\partial w}$ can be calculated using the non-linear function that replaces the multiplication in the original function. For instance, using the result from 
Section 5.2 (Equation 36):

$$
\begin{gathered}
=\beta w_{1 j} \sqrt{2\left(p_{1}-V_{T o}\right)^{2}-w_{1 j}^{2}} \quad \text { and } \\
\frac{\partial g}{\partial w_{11}}=\beta \sqrt{2\left(p_{1}-V_{T o}\right)^{2}-w_{1 j}^{2}}\left[1-\frac{w_{1 j}^{2}}{2\left(p_{1}-V_{T o}\right)^{2}-w_{1 j}^{2}}\right] .
\end{gathered}
$$

All the parameters necessary to calculate $\frac{\partial E}{\partial w}$ have been determined and can be used directly in Equation 19 and Equation 20.

VLSI cascade-correlation now incorporates limited numerical precision weight updates and non-linear synapses for training the synapses connected to the output units. All that remains is to incorporate the non-linear synapses into the hidden-node preparation portion of the algorithm.

In this stage, the correlation between the candidate hidden-unit's output and the LMS error is maximized. Recall the correlation function:

$$
C=\sum_{o}\left|\sum_{p}\left(y_{p}-\bar{y}\right)\left(E_{o p}-\bar{E}_{o}\right)\right| .
$$

The new $\frac{\partial C}{\partial w}$ expression can be derived in the same way $\frac{\partial E}{\partial w}$ was derived. Noting that the LMS error remains a constant during this phase of the training, it is found that $\frac{\partial C}{\partial w}$ is given by:

$$
\frac{\partial C}{\partial w}=\frac{\partial C}{\partial y} \frac{\partial y}{\partial g \partial w}=\sum_{o} \sum_{p} \operatorname{sgn}\left(E_{o p}-\vec{E}_{o p}\right)\left(\frac{\partial}{\partial g} f\right) p_{s p} \frac{\partial g}{\partial w}
$$

This works out to the same result as the one derived from the prototype cascade- 
correlation algorithm, with the addition of the $\frac{\partial g}{\partial w}$ term, which is implementation-specific and given in Equation 47 for the implementation of Chapter 5 .

Besides these new equations for minimizing the error, maximizing the correlation, and updating the weights, the procedure for applying the cascade-correlation algorithm remains unchanged. This aigorithm is a generalization of the cascade-correlation because it allows the implementation of a more complicated neuron transfer function.

\subsection{Justification of VLSI Cascade-Correlation}

To fully evaluate the VLSI cascade-correlation algorithm, the algorithm must be applied to a number of real-world applications using different configurations for the neuron/ synapse sub-circuits. However, possible advantages of the new algorithm can also be adequately demonstrated without such an in-depth analysis.

Firstly, as shown by Hoehfeld and Fahlman, $[1]$ the VLSI cascade-correlation algorithm successfully compensates for the limitations of the prototype's limited-precision weight updates by incorporating additional hidden units into the ANN. Hoehfeld and Fahlman found that the network is still be able to learn and generalize when the precision of the weight updates is less than eight bits.

Additionally, the VLSI algorithm compensates for the prototype algorithm's inability to perform non-linear synapse operations by adiling an extra term, $\left(\frac{\partial g}{\partial w}\right)$, to both the derivative of the correlation (Equation 49) and to the derivative of the error equations (Equation 45). If a linear (traditional) synapse is used in the new algorithm in place of the 
non-linear synapse, this term becomes the input signal, and the new correlation and error equations reduce to the equations of the prototype algorithm (Equations 22 and 15 respectively). Further, since the contribution of the non-linearity to the correlation and error equations is through a multiplication, the deviation from the results of the prototype algorithm only iecomes large as the non-linearity in the synapse becomes large. For small non-linearities, the modifications are minor, but may contribute positively to the final stages of the learning process, where the differences become more critical. ${ }^{[19]}$ If the synapse implementation is chosen to be a function that approximates a multiply, as opposed to an arbitrary non-linear function, then the approximation should rake little difference to the learning properties of the network.

To further justify the addition of the non-linear synapse, some simulations were performed using the circuits of Chapter 5. Figure 30 contains the synapse output for varying input voltages, given a fixed weight value in the synapse. Both the integrated non-linear synapse and the output of a corresponding linear synapse are showr. Using traditional cascadecorrelation, the non-linear function would be exhibited by the synapses integrated on the IC, but they would be modelled by the linear function of Figure 30 . Only for a limited range of input values would the prototype algorithm correctly estimate the synapse output In the VLSI algorithm, the synapse transfer function is incorporated, thus allowing the algorithm to more accurately estimate the synapse output value, and consequently improve the leaming properties.

Similarly, in Figure 31, two neuron outputs are shown - one incorporating the linear synapse, the other incorporating the non-linear synapse. The differences are most marked 
near the knees of the curve. These differences are important since the error and correlation functions would be markerly inaccurate in these regions, leading to an incorrect weight update value. Once again, VLSI cascade-correlation compensates for this inaccuracy since the output assumed by the learning algorithm more closely matches that of the circuit.

Finally, the modifications made to the prototype algorithm affect only those components of the prototype that relate to the neuron model. The method by which the correlation and the error are calculated - which are responsible for the learning properties of the network - remain unchanged.

The VLSI cascade-correlation algorithm thus preserves the learning properties of the prototype cascade-correlation algorithm, while allowing the neural network hardware to be more closely modelled. 


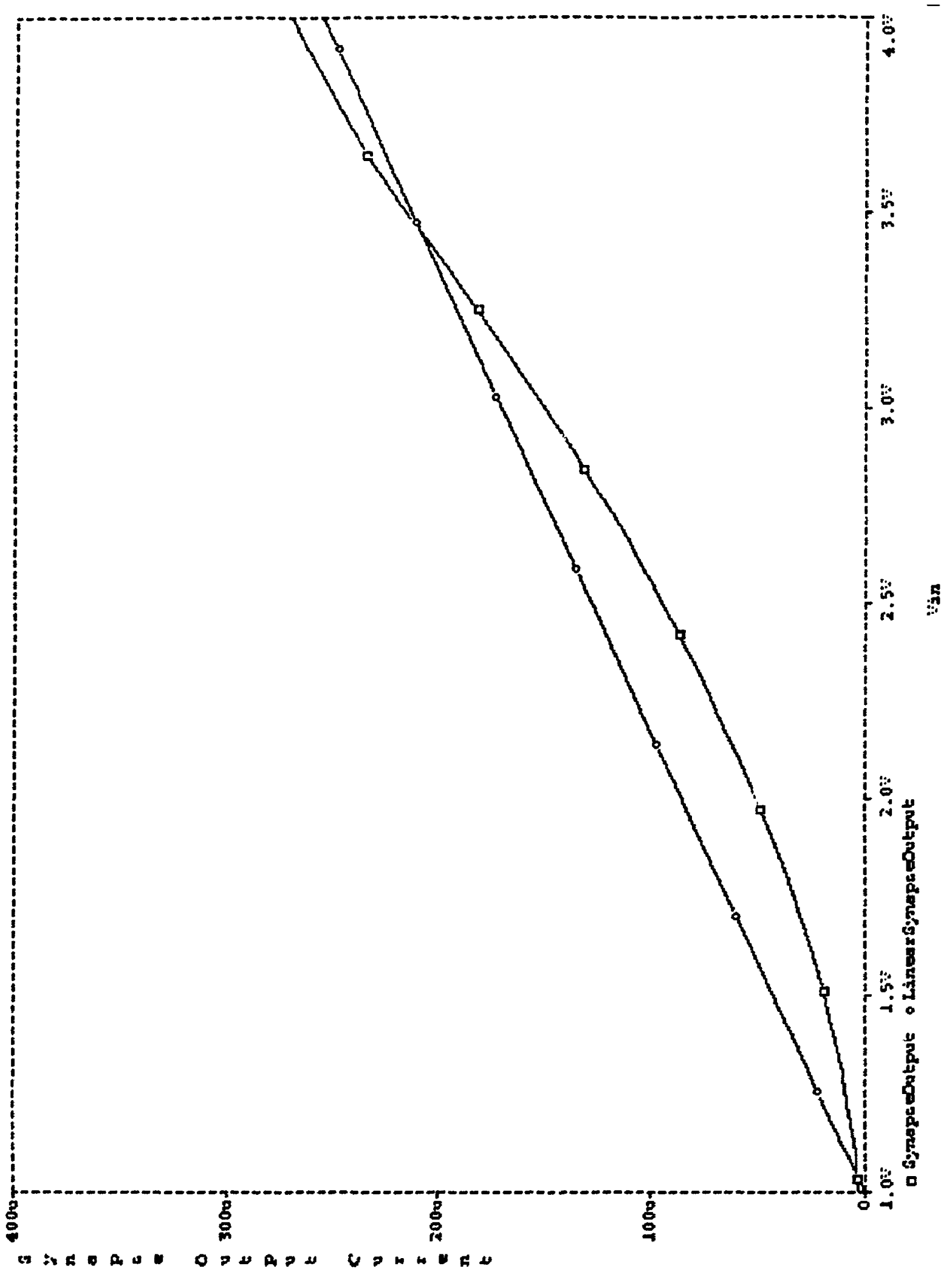

FIGURE 30 : Integrated and Lineur Synapse Responses. 


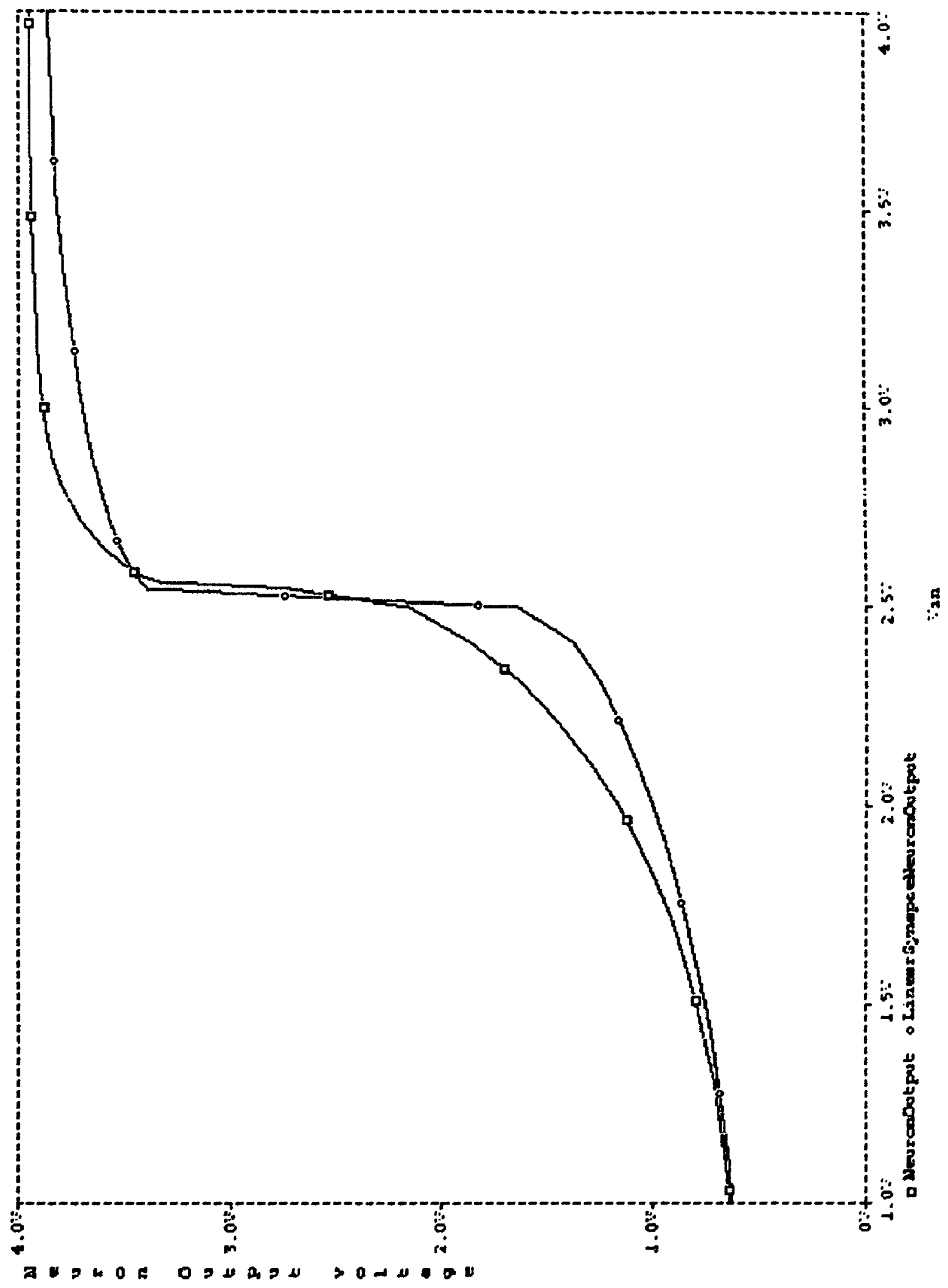

FIGURE 31 : Neuron Output Assuming Both Linear and Non-Linear Synapses 


\subsection{Chapter Summary}

A new training algorithm was developed for the training of neural networks implemented using specialized hardware. VLSI cascade-correlation - based directly upon cascadecorrelation - includes the facility to use limited precision (or limited dynamic range) weights. Further, the synapse operation is not limited to a true multiply, thus allowing more accurate correlation between neural networks implemented in analcg hardware (where a true multiply may not necessarily be achieved) and the software training algorithm.

The algorithm was then verified by illustrating how the learning properties of the traditional cascade-correlation algorithm are preserved in the VLSI algorithm, since the operational principles remain unchanged. The correlation and error functions are modified only through the inclusion of the more complex synapse model. Additionally, the new algorithm was shown to be a generalization of the original, reducing to the original when the traditional neuron transfer function is used.

Further, comparisons of the behavior of the integrated network and the network assumed by the prototype cascade-correlation algorithm demonstrate that the prototype cascadecorrelation algorithm does rot accurately model the integrated network. 


\section{Chapter 7. Conclusions}

The objective of this thesis was to evaluate the feasibility of implementing neural networks in VLSI.

First, a review of neural networks (Chapter 2) was presented followed by an investigation of two supervised learning algorithms used to train neural networks (Chapter 3).

Two learning algorithms were examined and compared - the backpropagation algorithm and the cascade-correlation algorithm. The numerical precision requirements of backpropagation were found to be too great for the cost-effective implementation of hardware-based ANNs. Additionally, the network architecture for a network trained using backpropagation is empirically chosen. By contrast castade-correlation has lower numerical precision requirements and automatically generates the network during training. Cascade-correlation was thus found to be a more suitable algorithm for the implementation of hardware-based ANNs.

This work then evaluated the potential of different technologies for implementing costeffective large neural networks (Chapter 4 ). The technologies examined include softwarebased neural networks, neural networks based on discrete hardware, and various

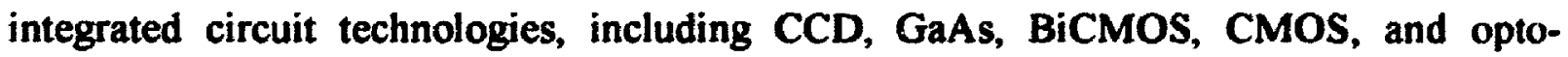
electronics. Although neural networks simulated in software are more flexible in terms of their re-programmability and the precision of their weight values, they lack sufficient parallel structure to allow networks to run efficiently. Discrete hardware, on the other 
hand, is inefficient because of the high level of interconnections required. Of the integrated circuit technologies, only CMOS allows the cost-effective implementation of large neural networks and non-volatile weight storage.

Both CMOS digital and analog implementation strategies were then compared (Chapter 4). Analog circuitry was found to be the best-suited technology because it allows the use of very simple components to implement each of the required functions.

Based on previous research of analog implementations of neural networks, a new neural architecture, similar to that proposed by Bibyk and Ismail, ${ }^{[21]}$ was presented (Chapter 5). This architecture consists of a floating-gate differential pair to implement the synapse, a linear current-to-voltage converter to implement the soma, and a transconductance amplifier to implement the activation function.

While anaiog CMOS is a good implementation medium for creating large neural networks, the simple building blocks used to create these networks do not necessarily model traditional artificial neuron behavior. This was illustrated by the models developed for the new architecture in Chapter 5. To overcome this shortcoming, the main contribution of this work, a new learning algorithm based on cascade-correlation, VLSI cascade-correlation, was proposed (Chapter 6).

VLSI cascade-correlation is a generalization of the traditional cascade-correlation algorithm. The new algorithm can train networks constructed with synapses that display limited dynamic range, and that do not accurately implement the multiplication function. Most researchers attempt to implement neurons and synapses that match the ideal neuron/ 
synapse transfer functions as closely as possible - thus complicating the network design The motivation for doing this lies in the limitaticns of the learning algorithms used to train the networks - they cannot accommodate a non-linear synapse. Training cannot converge if the transfer functions implemented in the network do not match the transfer functions implemented in the learning algorithm. The new learning algor, im allows the circuit transfer functions to be included.

The new algorithm was justified (Chapter 6) by comparing the transfer function of the neuron implemented in hardware to that implemented by the training function.

\subsection{Suggestions For Further Study}

In this work, the building blocks for the implementation of a cost-effective ANN - a new architecture and an associated training algorithm - were presented. One suggestion for further study is to use these building blocks to construct a VLSI-implemented ANN for application to real-world problems.

A second suggestion for further study is to pursue additional improvements to the cascade-correlation algorithm. The network architecture created by the cascadecorrelation algorithm was deemed adequate for this work because the algorithm defines the network configuration. A limitation of the algorithm in its current form is that it generates very deep networks which are undesirable due to resultant delay. In fact, a cascade-correlation network has as many hidden layers as hidden units. By contrast, networks based on backpropagation ustially contain less than four hidden layers. Sjøgaard from Aarhus University has proposed an alternative to cascade-correlation, ${ }^{[33]}$ called the 
second-order cascade-correlation algorithm, which adds hidden units to a single hidden layer instead of creating single neuron layers. The resulting networks are more shallow than cascade-correlation networks. The algorithm is identical to cascade-correlation except for network topology. Although this work is quite promising, this new algorithm has not yet been comprehensively evaluated.

A second enhancement to the cascade-correlation algorithm has been proposed by IBM. ${ }^{[34]}$ IBM's algerithm limits the fan-in of every hidden neuron. Surprisingly, the researchers found the limited network displayed superior generalization abilities than the cascade-correlation algorithm. The reduced fan-in network allows better mapping of cascade-correlation to SIMD-parallel neurocomputers (Single Instruction Multiple Data), as well as simpler VLSI implementation, since both routing and the number of synapses are reduced. The number of weights required to solve the two-spiral problem was reduced by a factor of two.

The work presented in this research can be combined with Sjogaard's, and Klaggees' to produce another algorithm that can be used to train VLSI-implemented neural networks. 


\section{References}

[1] Verleysen, M., and Jespers, Paul, "An Analog VLSI Implementation of Hopfield's Neural Network," IEEE Micro, December 1989, pp. 46-55.

[2] Hinten, Geoffrey, Neural Networks for Industry - Course notes, December 1992.

[3] Fukushima, Kunikiko, "A Neural Network for Visual Pattern Recognition," Computer, March 1988, pp. 65-75

[4] Mead, Carver, sinalog VLSI and Neural Systems, Addison Wesley, 1989.

[5] Rosenblatt, F., Principles of Neurodynamics, Spartan Books, 1959.

[6] Minsky, M. and Papert, S., Perceptrons: An Introduction to Computational Geometry, MIT Press, 1969.

[7] Müller, B., and Reinhardt, J., Neural Networks - An Introduction, Springer-Verlag. 1990.

[8] Fahlman, S. E., and Lebiere, C., "The Cascade-Correlation learning architecture," Advances in Neural Information Processing Systems 2, D.S.

Touretzky, Ed. 1990, pp. 524-532.

[9] Kröse, Smagt, An Introduction to Neural Networks, Fifth Edition, January 1993.

[10] DARPA, Neural Network Study, AFCEA International Press, 1988.

[11] Hoehfeld, M., and Fahlman, S. E., "Learning with Limited Numerical Precision Using the Cascade-Correlation Algorithm," IEEE Trans. on Neural Networks, July 1992, pp. 602-611.

[12] Murray, A., and Edwards, P., "Synaptic Weight Noise During Multilayer Perceptron Training: Fault Tolerance and Training Improvements," IEEE Trans. on Neural Networks, July 1993, pp. 722-725.

[13] Fahlman, S. E., "Faster-learning variations on back-propagation: An empirical study," Proc. 1988 Connectionist Models Summer School, pp. 3851.

[14] Lansner, J., and Lehmann, T., "Analog CMOS Chip Set for Neural Networks with Arbitrary Topologies," IEEE Trans. on Neural Networks, May 1993, pp. 441- 444.

[15] Alvarez, A. R. ed., BiCMOS Technology and Applications, Kluwer Academic Publishers, 1989. 
[16] Durfee, D. A., and Shoucair, F. S., "Comparison of Floating Gate Neural Network Memory Cells in Standard VLSI CMOS Technology," IEEE Transactions on Neural Networks, May 1992, pp. 347-353.

[17] Thomsen, Axel, and Brooke, Martin, "A low-cost application-specific neural network implementation with floating gate weights," The International Joint Conference on Neural Networks - Conference Proceedings, Volume 2, 1992, pp. II-565-II-570.

[18] Hoekstra, J., “(Junction) Charge-Coupled Device Technology for Artificial Neural Networks," VLSI Design of Neural Networks, Ramacher, Ulrich, and Rückert, Ulrich, ed., 1991, pp. 19-45.

[19] Lont, Guggenbühl, "Analog CMOS Implementation of a Multilayer Perceptron with Nonlinear Synapses," IEEE Trans. on Neural Networks, May 1992, pp. 457-465.

[20] Sharma, R., "VLSI Electronic Neural Networks", Submicron Integrated Circuits, ed. R. K. Watts, 1989, pp. 413-433.

[21] Bityk, Steven, and Ismail, Mohammed, "Neural Network Building Blocks for Analog MOS VLSI," Analog IC Design: the current-mode approach, C. Toumazou et. al., ed. 1990, pp.597-615.

[22] Arima, Yutaka, et. al., "A 336-Neuron, 28K-Synapse, Self-Learning Neural Network Chip with Branch-Neuron-Unit Architecture," IEEE Journal of Solid-State Circuits, November 1991, pp. 1637-1644.

[23] Nelson, M. M., "A Practical Guide to Neural Networks," Addison-Wesley, 1991.

[24]Mueller, et.al., "A General Purpose Neural Network," The International Joint Conference on Neural Networks - Conference Proceedings Volume 2, 1989, pp II-177 - II-182.

[25] Tsividis, Y.P., and Anatassiou, D., "Switched-Capacitor Neural Networks," Electronic Letters, 1987, pp. 958-959.

[26] Martin, K., and Sedra, A. S., "Strays-Insensitive Switched-Capacitor Filters Based on the Bilinear z-transform," Electronic Letters, June 1979, pp. 365-366.

[27] Allan, P., and Holberg, D., CMOS Analog Circuit Design, Holt, Reinhart and Wilson, 1987.

[28] Lee, Bang and Sheu, Bing, "General Purpose Neural Chips with Electrically Programmable Synapses and Gain-Adjustable Neurons," IEEE Journal of Solid-State Circuits, September 1992, pp. 1299-1302. 
[29] Vittoz, Eric, "Micropower Techniques," Design of MOS ILSS Circuits for Telecommunications, Tsividis et. al., ed., pp. 104-144.

[30] Shousha, A. H. M., and Aboulwafa, M., "A Generalized Tanh Law MOSFET Model and Its Applications to CMOS Inverters," IEEE.Journal of Solid-State Circuits, February 1993, pp. 176-179.

[31] Choi, Joongho, and Sheu, Bing J., "VLSI Design of Compact and High-Precision Analog Neural Network Processors," IEEE Joint Conference on Neural Networks, 1992, v.II, pp. II-637-II-641.

[32] Caviglia, Daniele D., et. al., "Analog VLSI Systems for Neural Adaptive Architectures," Neural Networks Concepts, Applications, and Implementations Volume II, Paolo Antognetti et. al., ed. 1991, pp. 207-223.

[33] Sjøgaard, Steen, A Conceptual Approach to Generalization in Dynamic Neural Networks, Ph.D. Thesis - Computer Science Dept., Aarhus University, 1991.

[34] Klaggees, Henrik, and Soegtrop, Michael, Limited Fan-in Random Wired Cascade-Correlation, unpublished. 

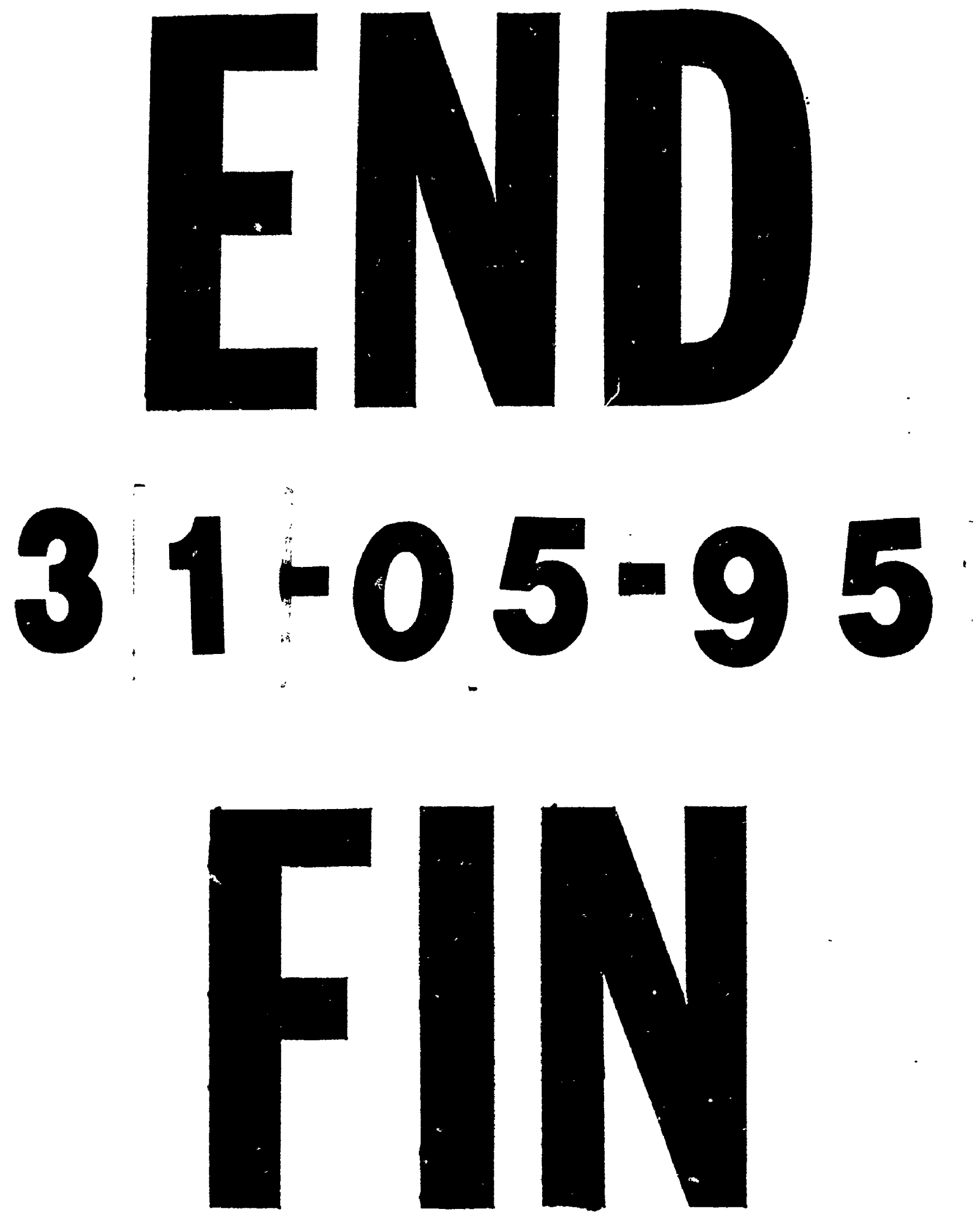

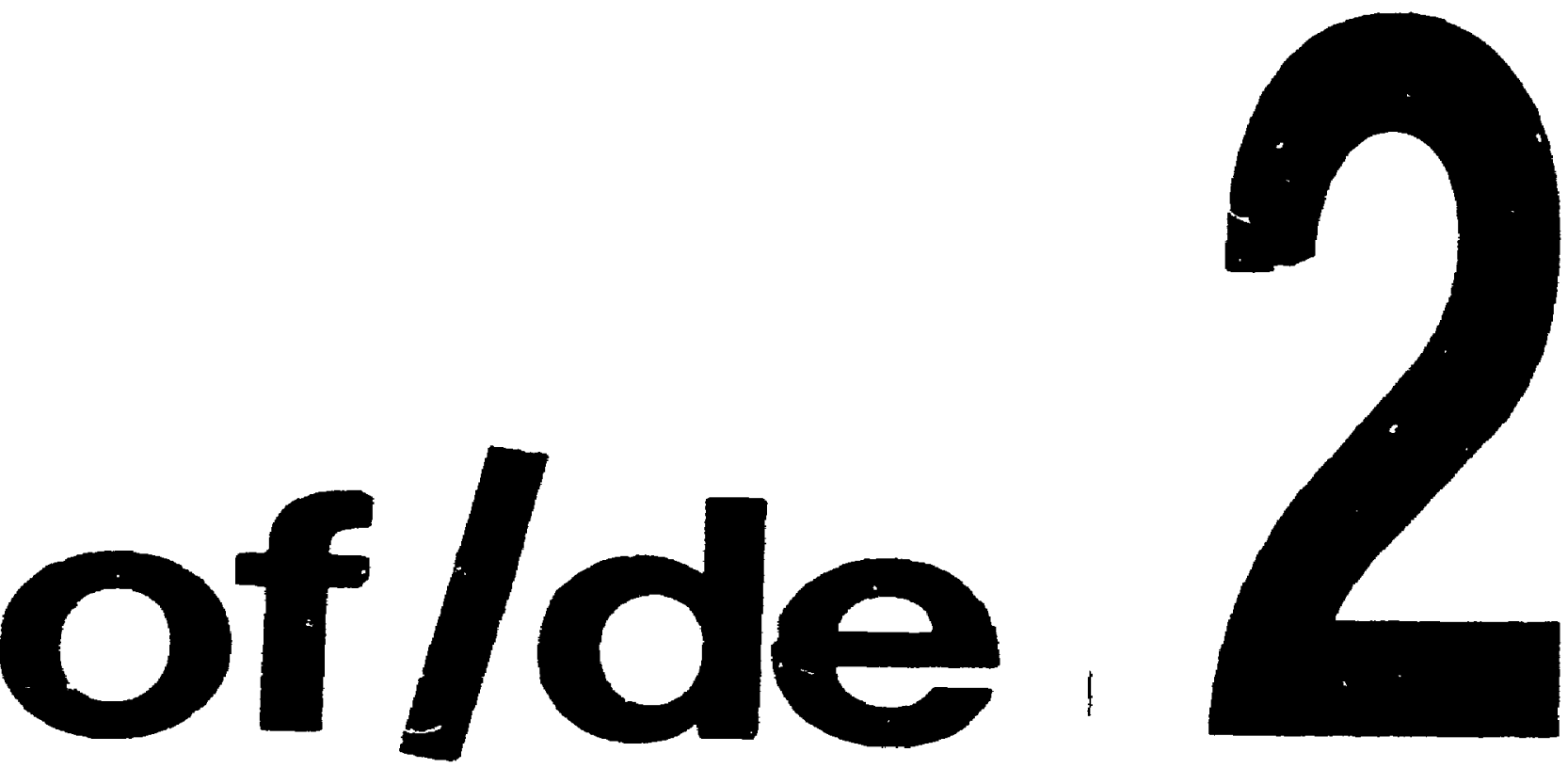

PM-1 31/2"x4" PHOTOGRAPHIC MICROCOPY TARGET NBS 1010a ANSI/ISO \#2 EOUIVALENT

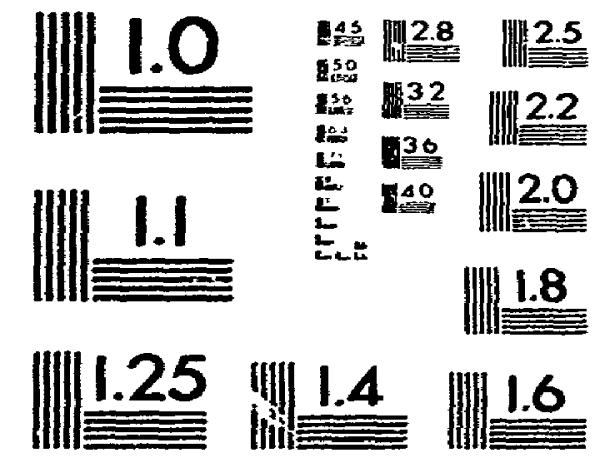

PRECISIONSM RESOLUTION TARGETS 